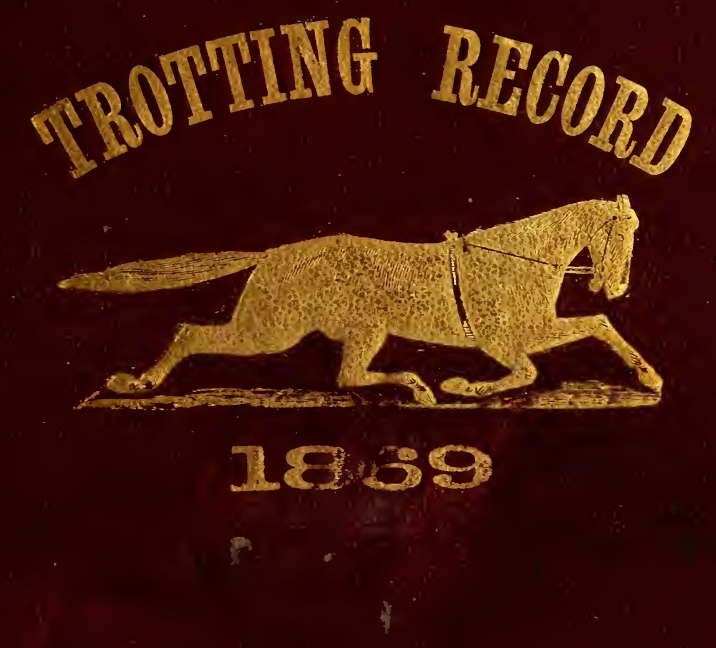




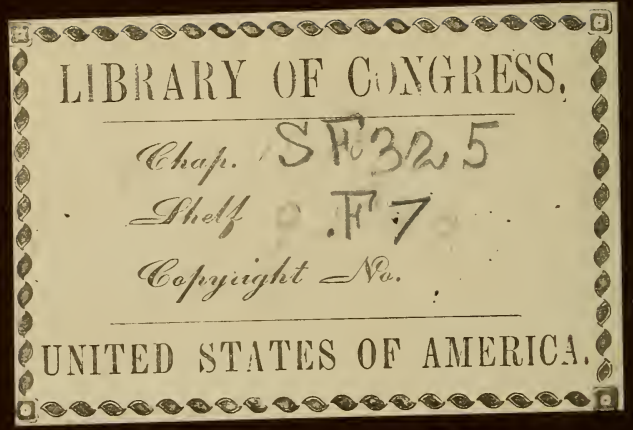





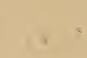





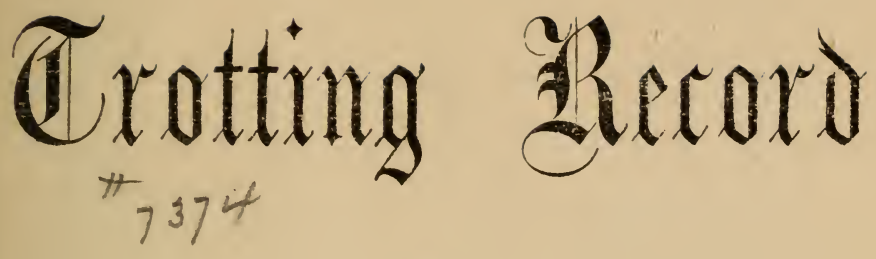
FOR

\section{9 .}

CONTAINING A COMPLETE AND RELIABLE RECORD OF ALL, THE

TROTTING EVENTS OF THE PAST SEASON, COMPILED AND ARRANGED WITH GREAT CARE, AND RESPECTFULLY , DEDICATED TO

AMERICAN TURFMEN.

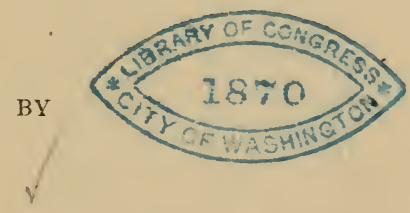

\section{FRANCIS \& HALEY,}

NEW YORK. 


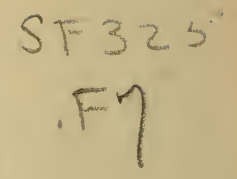

Entered according to Act of Congress, in the year 1870 , by FRANCIS \& HALEY,

in the Cierk's Office of the District Court, for the Southern District of New York. 


\section{THIS WORK}

IS, BY PERMISSION, RESPECTFULLY INSCRIBED TO

Charles J. Foster, EsQ.,

OF "THE SPIRIT OF THE TIMES,"

TO WHOM THE AUTHORS ARE DEEPLY INDEBTED, AND

BY WHOM ITS COMPOSITION WAS

FIRST SUGGESTED. 



\section{INTRODUCTION.}

The Trotting Record was first suggested by the frequent demand of American Turfmen for an authentic record of the performances of the Trotting Horse of America.

The advantages of such a record, condensed into a single volume of convenient size, will be appreciated by all who have heretofore been forced by circumstances to refer the performances of any horse to the Editors of a sporting paper, whose decisions it is true are authority, but to whom reference is frequently a matter of weeks of time, and certainly of some expense, besides being a source of trouble to the paper referred to, the kindness of which in determining such matters is fully appreciated.

It is proposed to record in the present work not only those races which have taken place on the principal TrotTING TRACKS of the country, where records are preserved, but those of Agricultural Societies; on whose tracks some of the most noted trotters of the United States have trotted some of their finest races. (Flora Temples's famous time at Kalamazoo, Mich. for instance.) These races come within the legitimate province of a Trotting Record, as portions of the present work, and are fully authenticated as necessary to a complete and reliable book of this nature 
The scope of the Trotting Record is not of sufficient limit to give, In Extenso, the details of each of the races of even the most celebrated of the courses mentioned. This would necessitate a volume of vastly more bulk than would be deemed convenient, in fact it would require many volumes of larger size than is here published. The genuine record is all that is required by horsemen, and this, it is believed will here be found, compressed within a few pages, with an index making a reference easy and certain.

It is the intention of the editors of the Trotting Record to continue its publication from year to year, as each season furnishes its material.

Officers of Agricultural Societies and proprietors of Trotting Tracks are respectfully requested to act as agents for the circulation and sale of the Trotting Record. They are also requested to forward to the editors full and reliable accounts of future trots at their respective meetings, and of matches worthy of notice in such a work, to be incorporated in future editions to fuliy meet the requirements of so valuable a book to American Turfmen.

FRANCIS \& HALEY.

NEW YORK. 


\section{TROTTING RECORD}

FOR 1869.

\section{MAY 25TH, 1869.}

\section{PROSPECT PARK-Fair Grounds, Gravesend, I. I.}

TUESDAY, May 25th.-Purse $\$ 500$, for all horses that have never beaten 2:45. Mile heats, best three in five, in narness. $\$ 350$ to first, $\$ 100$ to second, $\$ 50$ to third horse:

Dan Mace enters b m Lydia Thompson ..........1 1

Dan Pfifer " br m No Name..............

John Murphy “ b m Lady Ella.. . ........... $33 \quad 3$

E. Ward " b g Trenton(formerly Defiance).2 $4 \quad 4$

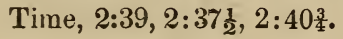

MAY $27 \mathrm{TH}, 1869$.

PROSPECT PARK.-Fair Grounds, Gravesend, L. I.

Thursday, May 27th.-Purse $\$ 500$, for all horses that have never beaten 2:37. $\$ 350$ to first, $\$ 100$ to second, $\$ 50$ to third horse. Mile heats, best three in five, in harness.

E. Ward

Dan Mace

John J. Hornbeck

Wm. Dall

John Murphy

Alden Goldsmit :

J. Cudney

J H. Whitson

Owner enters $g$ g Mrrcer.......... 11

" b m Lydia Thompson...4 $4 \quad 2$

" s g Agilis.............2 $2 \quad 3$

“ blk s Mike Norton........3 34

" b m Lady Franklin.....5 55

". $8 \mathrm{~g}$ Hunter ...........dr.

6. b m Atlanta.......... dr.

" s g Clarence............dr.

6 br g Sir John........... dr.

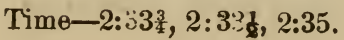


MAY 29TH, 1869.

\section{PROSPECT PARK.-Fair Grounds.}

MAY 29th.-Premium $\$ 1,000$. $\$ 700$ to first, $\$ 200$ to second, $\$ 100$ to the third horse. Mile heats, best three in five, in hari ers.

M. Roden

G. W. Ferguson

Dan Mace

F. J. Nodine

W. Borst

Alden Goldsmith

James McKee

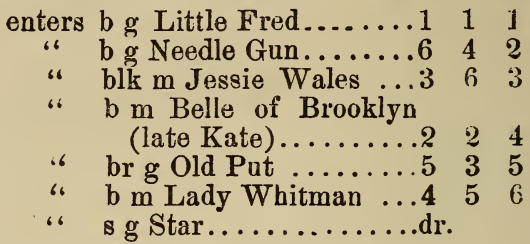

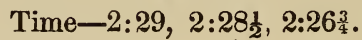

MAY 29TH, 1869.

\section{PROSPECT PARE. - Fair Grounds.}

MaY 29th.-Purse $\$ 5,000$, for all horses except Mountain Boy and Lady Thorn. $\$ 3,000$ to first, $\$ 1,500$ to second, and $\$ 500$ to third horse. Mile beats, best three ia five, in harness.

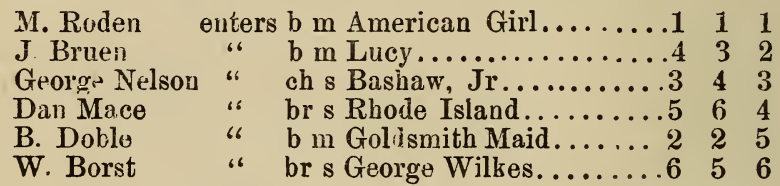

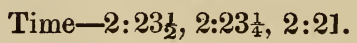

JUNE 1sT, 1869.

UNION COURSE, L. I.

JUNE 1st. - Purse $\$ 500$. $\$ 300$ to first, $\$ 125$ to second and $\$ 75$ to the third horse. Mile heats, best three in five, in harness.

John Murphy enters br m Lady Sears.......... 1

A. Patterson

Dr. Ogle

Dan Mace

J. Chambers

M. Roden

G. Brown

A. Johnson

W. H. Saunders

Owner

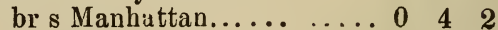

ch $\mathrm{g}$ Pet $\ldots \ldots \ldots \ldots \ldots \ldots$. 2 2 3

b m Lydia Thompson. . . . . .4 34

b g Twist..............dis

blk g Eight Bells..........dis

br m Lady Collyer........dr

br g N. B. Hayes..........dr

$\mathrm{s} \mathrm{g} \mathrm{H}$. W. Chittenden......dr

s g Frank..............dr 
F. J. Nodine enters $\mathrm{b} \mathrm{m}-\ldots \ldots \ldots \ldots \mathrm{dr}$ Wm. Borst " b s Hary Clay...........dr Wm. Lovell " b b g Young Commodore... dr D. Pfifer “ $\quad$ g g R. R. Stuyvesant......dr S. Hoagland " g g Prospect................ dr E Perrin " b g Ed Lewis.............dr Geo. W. Nelson b c Reverdy Johnson........dr H. Howe

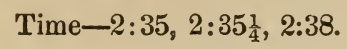

JUNE 1ST. 1869.

UNION COURSE, I. I.

Purse $\$ 300$. $\$ 450$ to first, $\$ 225$ to second, $\$ 125$ to the third horse. Mile heats, best three in five in harness.
A. Johnson enters
O. Goodwin "“
br \& Geo. M. Patchen, Jr_...1 11
T. Canary
W. Dall
br g Sir John............. 3 \%
J. Murphy
b g No Name.............
R. Walters " blk g W. M. Tweed .........dr
J. H. Whitson" "
W. Borst
s g Clarence..............dr
G. N. Ferguson
g g Pat Molloy .............dr
A. Patterson "
G. Nelson
br g Needle Gun...........dr
b g Keystone............dr
g g Warsaw............. dr

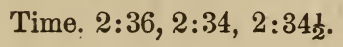

JUNE 2ND, 1869.

\section{UNION COURSE, I. I.}

June 2.-Purse $\$ 2,500 . \$ 1,400$ to first, $\$ 500$ to second, $\$ 350$ to third, $\$ 250$ to the fourth horse. Mile heats, best three in five, in harness.

W. Lovell enters
N. Holcomb "6
B. Doble
D. Pfifer
W. Borst "“
G. W. Nelson “"
M. Roden "6

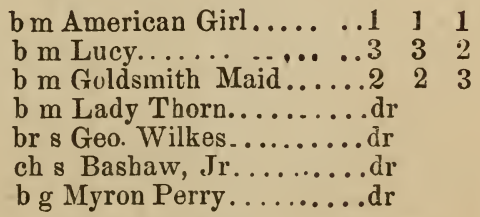

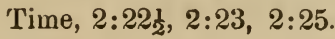


JUNE 2D, 1869.

\section{UNION COURSE, I. I.}

June 2d.--Purse $\$ 600$. $\$ 300$ to the first, $\$ 200$ to second, $\$$ Juo to the third horse, for horses that never trotted better than $2: 45$. Mile heats, best three in five, in harness.

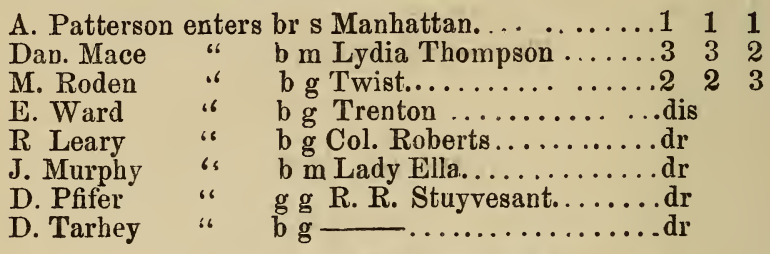

Time, $2: 34 \frac{1}{2}, 2: 36,2: 37 \frac{1}{2}$.

JUNE 3D, 1869.

\section{UNION COURSE, I. I.}

JunE 3d.-Purse $\$ 500 . \$ 300$ to first, $\$ 125$ to second, $\$ 75$ to the third horse, mile beats, best three in n̂ve, in harness, for all horses that have never beaten 2:50.

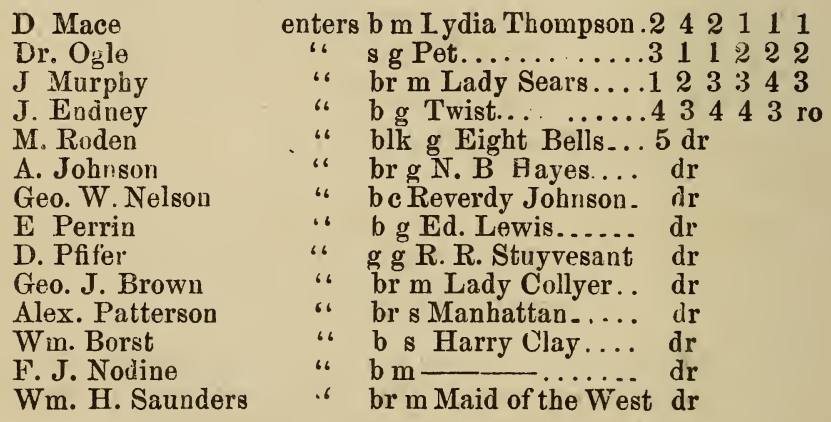

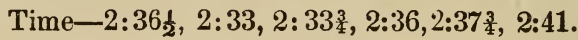

JUNE 3D, 1869.

\section{UNION COURSE, L. I.}

JUne 3d.-Purse $\$ 1,000$. $\$ 600$ to first, $\$ 300$ to second, $\$ 100$ to the third horse, for horses that have never beaten 2:31. Mile heats, best three in five, in harness. 


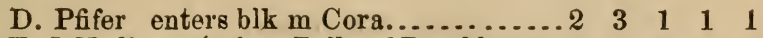

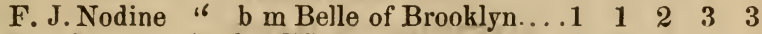
Dan Mace " b g Western New York. $3 \quad 2 \quad 3 \quad 2 \quad 2$ M Roden " b g Little Fred.......... dr

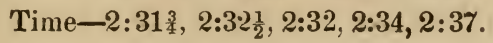

\section{JUNE 4TH, 1869. \\ UNION COURSE, L. I.}

June 4th.-Purse $\$ 750$. $\$ 400$ to first, $\$ 225$ to second. $\$ 125$ to third horse. Mile heats, best three in five, in harness, for all horses that have never beaten $2: 40$.

E. Ward

Geo. W. Nelson

M. Roden

Dan Mace

A. C. Whitson

J. Murphy

J. Campbell

J. H. Whitson

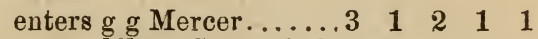

" blk s Star of the

West........

" b b g Dresden....

" ch m Louise.....4 dr.

“ b g Star......... dr.

" b m Lady Frauklin dr.

" $\quad$ b g Jim......... är.

" s gelding Clarence. dr.

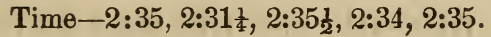

\section{JUNE 4TH, 1869. \\ UNION COURSE, I. I.}

JUNe 4th.-Match for $\$ 1,000$. Mile heats, $b$ st three in five, in harness.

M. Roden enters b g Young Commodore......... 11

J. Murphy " b g Red Rover..............2 2 dr.

Time-2:43, 2:43.

JUNE 8TH, 1869.

- DEXTER DRIVING PARK.

June 8 th - Purse $\$ 600$. $\$ 400$ to first, $\$ 150$ to second, $\$ 50$ to the third horse, for horses that have never beaten 2:30. Mile heats, best three in five, in harness.

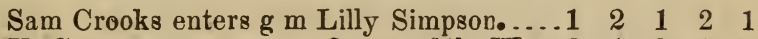

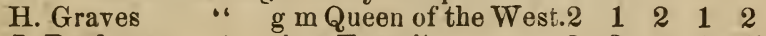

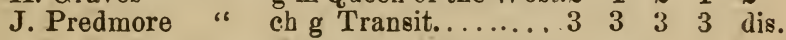

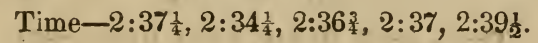


JUNE 9TH, 1869.

\section{RIVERSIDE PARK.}

JUNE 9th -Purse $\$ 4,000$. $\$ 2,000$ to first, 1,000 to secnnd, $\$ 600$ to third, $\$ 400$ to the fourth horse, for all horses that have never beaten $2: 30$.

J. Turner enters b m Fanny Allen......... $1 \begin{array}{llll}1 & 2 & 1 & 1\end{array}$

F J. Nodine " b m Belle of Brooklyn.....2 12 2 2

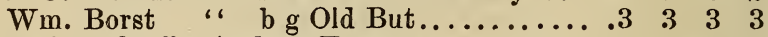

H. Woodruff “ b m Empress............. 4 4 $44 \quad 4$

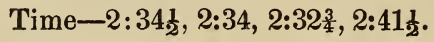

JUNE 9TH, 1869.

\section{FIVERSIDE PARK.}

June 9th.-Purse $\$ 500$. $\$ 350$ to first, $\$ 100$ to second, $\$ 50$ to the third horse. Mile heats, three in five, in harness, for horses that have never beaten three minutes.

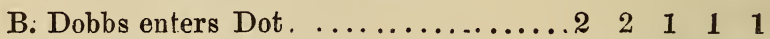

M. Roden " Jem Libby.....,...........

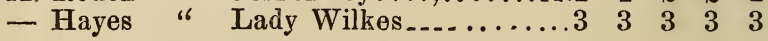

Time-2:40, 2:37 2:39, 2:393 $2: 41 \frac{1}{2}$.

JUNE 10TH, 1869.

RIVERSIDE PARK.

JUNE 10th.-Purse $\$ 1,500$. $\$ 800$ to first, $\$ 500$ to second, $\$ 200$ to third horse. Mile heats, best three in five, for horses that have never beaten 2:25.

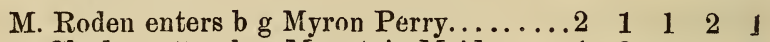

- Clark “ b m Muuntain Maid.......

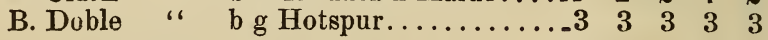

Time-2:31 $\frac{3}{4}, 2: 34,2: 34 \frac{1}{2}, 2: 35,2: 36$.

JUNE 10TH, 1869.

RIVERSIDE PARK.

June 10th.-Purse $\$ 700$. $\$ 400$ to first, $\$ 200$ to second, $\$ 100$ to third horse. Mile heats, three in five, for horses that have never beaten 2:40.

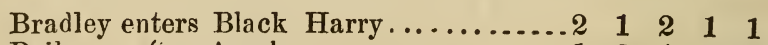

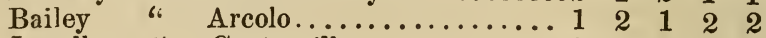

Jewell " Centreville.................

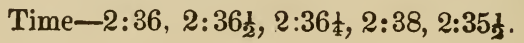


JUNE 10TH, 1869.

\section{DEXTER DRIVING PARK.}

JCNE 10th.-Purse $\$ 2,000$. Mile heats, three in five, in harness, for horses that have never trotted in public on a regular track, $\$ 1,200$ to first, $\$ 400$ to second, $\$ 250$ to third, and $\$ 150$ to the fourth horse.

J. S. Wolf enters g $g$ Joe Hooker............. 1

H. H. Yates " b m Kate Hazard.............. 2 2 2

J. Gallagher “ $\mathrm{g} \mathrm{m}$-atinet................. 2 2 $33 \quad 3$

J. Predmore “ \& g John Touly.............5 44

A.V. Brooking " ch s Lance................ dis

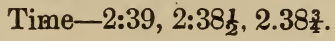

JUNE 11TH, 1869.

\section{RIVERSIDE PARK.}

JUNe 11 th. - Purse $\$ 2,500$. $\$ 1,400$ to first, $\$ 500$ to second, $\$ 350$ to tbird, $\$ 250$ to the fourth horse. Mile heats, best three in five, in harness.

M. Roden enters b m American Girl........... 1

B. Doble " b m Goldsmith Maid...

G. W. Nelson " ch \& Bashaw, Jr.............. 3 3 3

Dan Mace “ br s Rode Island ............ dr

Time-2:27, 2:26 $\frac{3}{4}, 2: 28 \frac{1}{2}$.

JUNE 11TH, 1869.

\section{DEXTER DRIVING PARK.}

JuNE 11th.-Purse $\$ 300$. $\$ 200$ to first, $\$ 100$ to the second horse, for horses that have never trotted better than $2: 35$. Mile heats, best three in five, in harness.

J. T. Haley enters $g$ g Sea Foam........... 11

Chas. Skinners " bs Tom Brady...........2 2 2

Time-2:391 $2: 43 \frac{1}{2}, 2: 45$.

JUNE 11TH, 1869.

HIGHLAND PARK COURSE, Newburgh.

June 11th.-Purse $\$ 2,000$. Mile heats, best three in five, in harness.

S. McLaughlin enters b g Mountain Boy,........ 1

J. McMann " b m Lady Thorn...........2 2

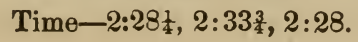




\section{JUNE 14TH, 1869. \\ MYSTIC PARK.}

JUNE 14th.-Purse $\$ 2,000$ for all horses that have never beaten 3:00. $\$ 1,000$ to first, $\$ 700$ to second, $\$ 300$ to the third horse. Mile heats, best three in five, to harness.
J. F. Phillips enters b s Danvers Boy........
John Jennings
B. Doble
b g Anthony Wayne......6
M. Roden
" s g Dot...............
W. Simmons " b g Jem Libby..........8
G. I. Dorsey " " g g Park Golddust.........9
Wm. McCarmachi b m Nina...............
W. Bain
Owner
"6 b g White Heels.........10
" $\mathrm{b}$ m Lizzie Packer......... -

$\begin{array}{lll}1 & 8 & 1 \\ 2 & 1 & 5 \\ 6 & 3 & 2 \\ 3 & 2 & 3 \\ 5 & 5 & 4 \\ 9 & 4 & 6 \\ 8 & 6 & 7 \\ 7 & 7 & \text { dis } \\ 4 & \text { ais } \\ \text { dis }\end{array}$

Time-2:301 $\frac{1}{2}, 2: 28 \frac{1}{2}, 2: 34 \frac{1}{2}, 2: 31 \frac{1}{2}$.

JUNE 15TH.

\section{MYSTIC PARK.}

JUNE 15th.-Purse $\$ 3,000$ for all horses that never have beaten $2: 25$. Mile heats, three in five to harness. $\$ 1,700$ to first, $\$ 1,000$ to second, $\$ 300$ to the third horse.

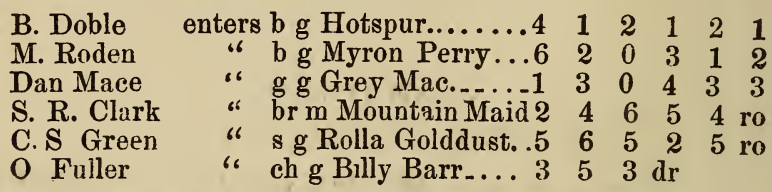

Time, $2: 25 \frac{1}{2}, 2.26 \frac{3}{4}, 2: 27 \frac{1}{4}, 2: 26 \frac{3}{4}, 2: 30,2: 30 \frac{1}{2}$.

JUNE 16TH, 1869.

IMYSTIC PARK.

JUNE 16th.-Purse $\$ 2,000$ for all horses that have nerer beaten 2:40. Mile heats, best three in five, to harness. $\$ 1,000$ to first. $\$ 700$ to second, $\$ 300$ to the third horse.

D. Pfifer enters br s Daniel Boone............ 1 1 1

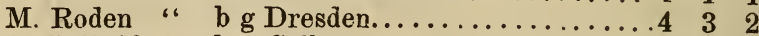

S. G. Reid “ b m Sally ................... $322_{2}$

Owner " b m Lizzie Packer................2 4 4 4

D. P. Bissell b g Wilson..................

D Mace “ s g Locust....................

W. S. Thomas s m Cariotta................. dis

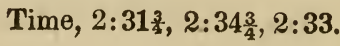




\section{JUNE 17TH, 1869. \\ MYSTIC PARK.}

JUNE 17 th. - Purse $\$ 2,000$, for horses that have never beaten 2:50. Mile heats, best three in five, in harness. $\$ 1,000$ to first, $\$ 7 \cup 0$ to second, $\$ 300$ to the third horse.

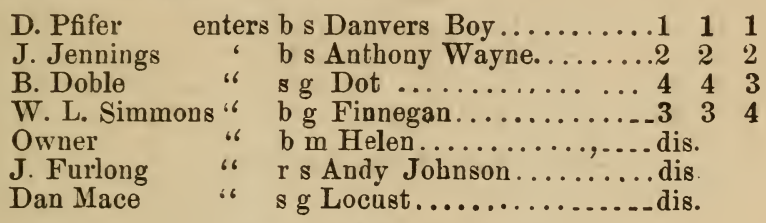

Time-2:33, 2:334, 2:28.

JUNE 17TH, 1869.

MYSTIC PARK.

JUNE 17th.-Purse $\$ 3,000$, for all horses that have never beaten $2: 30$. Mile heats, best three in five, in harness. $\$ 1,700$ to first, $\$ 1,000$ to second, $\$ 300$ to the third horse.

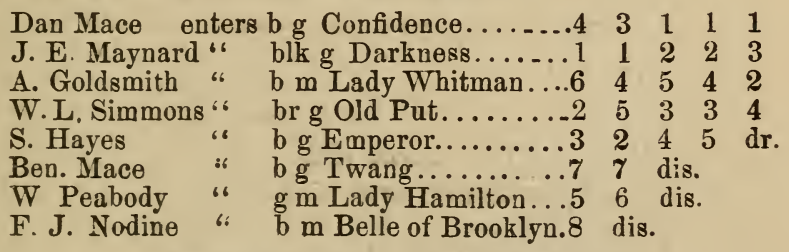

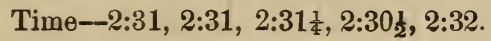

\section{JUNE 18TH, 1869. \\ MYSTIC PARK.}

JUNE 18th.-Purse $\$ 2,000$, for all horses that have never beaten 2:35. Mile heats, best three in five, in barness. $\$ 1,000$ to first, $\$ 700$ to second, $\$ 300$ to the third borse.
D. Mace
Owner
enters $\mathrm{w} g$ White Fawn...... 21132211
D. P. Bissell
6. Rosa Golddust..........3 4111.52
R. L. Flander
"6 b g Wilson.............1 $2444 \approx 3$
" b m Lady Ellis.......... 455553 r.o.
S R. Perkins " w g Black Harry........ $5322 \quad 344$ r.o.
W. H. Woodruff " blk g Black Walnut....dis.

Time-2:31 $\frac{1}{2}, 2: 32 \frac{1}{4}, 2: 34 \frac{1}{2}, 2: 33 \frac{1}{2}, 2: 35,2: 37$. 
JUNE 18TH, 1869.

MYSTIC PARK.

June 18th.—Purse $\$ 4,000$, for all horses. Mile heats, best three in five, in harness. $\$ 2,000$ to first, $\$ 1,500$ to second, $\$ 500$ to the third horse.
M. Roden
B. Doble
C. Chaplain
J. J. Bowen

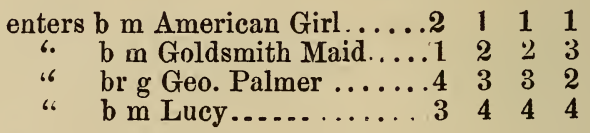

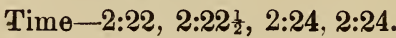

JUNE 22ND, 1869.

VALLEJO COURSE, Cal.

JUNE 22d.-Purse $\$ 200$. Mile heats, best three in five, in harness.

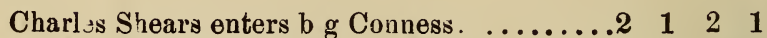

— White "sg G. F. Train........... 3 3 31 dis

— Squires " b m F Manderville.....1 2 dis

Time-2:45, 2:44, 2:43, 2:34.

JUNE 22ND, 1869.

WATERTOWN PARK.

June 22.-Purse $\$ 150$. $\$ 100$ to first, $\$ 35$ to second, $\$ 15$ to the third horse, for horses that have never trotted for money.

H. B. Griffen enters br $\mathrm{g}$ Brown Dick .......... 1

J. H. Phillips " " b m Linda................2 2

S. Lewis

B. Helmer

J. Snow

R. B. Adams

"6* ch m No Name............. 3 3 3

" g m Belle..............4 dis

$\therefore$ blk $m$ Phebe Robin......... dis

" br s Fessenden............ dr

Time-2:55 $\frac{1}{2}, 2: 50,2: 58$.

JUNE 22ND, 1869

WATERTOWN PARK.

JUNE 22.-Purse $\$ 300$. $\$ 200$ to first, $\$ 70$ to second, $\$ 30$ to the third horse, for horses that have never beaten 2:50. 
A. M. Harris enters s g No Name............. 11

J. H. Phillips " b g Billy (formerly Ace of

$\begin{array}{lllll} & \end{array}$

T. Ivנs « " g g Gray Jim.............2 2

A. Doran " b m Buteau................. dis

Time-2:4I $\frac{1}{2}, 2: 40,2: 39$.

JUNE 23RD, 1869.

WATERTOWN PARK.

June 23.-Purse $\$ 5 ? 0$. $\$ 300$ to first, $\$ 125$ to second, $\$ 75$ to the third horse, for horses that have never beaten 2:35.

E. Scott

J. Mott

A J. Feek

H. T. Isbam

A. M. Harris

A Clough

E. C. White

L. Dorsey

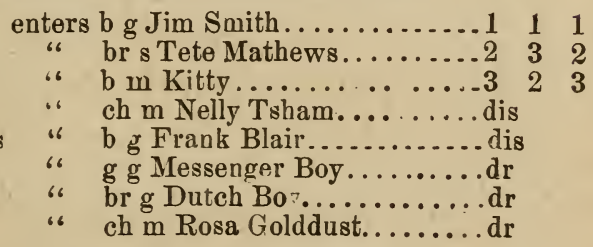

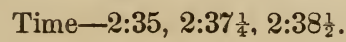

JUNE 23D, 1869.

\section{NARRAGANSETT PARK.}

JUNe 23.-Purse $\$ 1,500$, for all horses that have never trotted for money. Mile heats, best three in five, in harness, $\$ 1,000$ to first, $\$ 350$ to second, $\$ 150$ to the third horse.

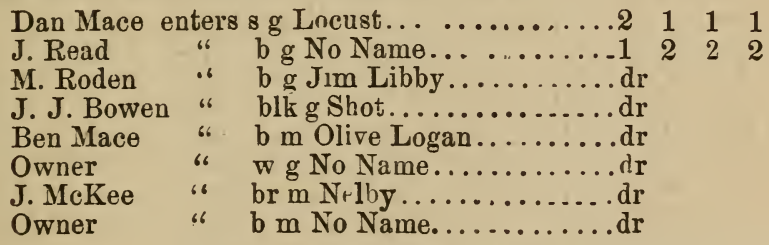

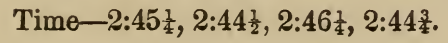

JUNE 23D, 1869.

\section{NARRAGANSETT PARK.}

JUnE 23d.-Purse $\$ 2,000$, for all trotting stallions. Mile heats, best three in five, in harness. $\$ 1,300$ to first, $\$ 500$ to second, $\$ 200$ to the third horse. 
Dan Mace enters br s Rhode Island.......... 1 G. W. Nelson "“ blk s Bashaw, Jr............2 2 W. L. Simmons" blk s Geo. Wilkes............dr.

Time, 2:30, 2,29, 2:28 $\frac{1}{2}$,

\section{JUNE 23RD, 1869. \\ BUCKEYE COURSE.}

June 23rd.-Club Purse \$200. Mile heats, best three in five. for horses that never beat 2:50.

H. B. Loder enters d g Greenback _... $\ldots \ldots \ldots 1 \quad 2 \quad 1 \quad 1$

J. P. Gilbert " ch g White Face, by Corbean.

H. Maher “ $\quad \begin{aligned} & \text { Dam by Lexington...2 } \\ & \text { gilot Madoc. By Pilot, }\end{aligned}$

H. W. Abbot “ b m Josie. By Abdallah, dam

by imp. Leviathan.......5 5500

G. M. Scott “ b g Cora Davis............ 34400

Time, 2:381 $2: 36 \frac{1}{2}, 2: 37 \frac{1}{2}, 2: 39 \frac{3}{4}$.

\section{JUNE 23RD, 1869. \\ BUCKEYE COURSE.}

JunE 23rd.-Club Purse $\$ 200$, for all horses that have never beaten 2:45. Mile heats, best three in five, in harness.

J. B. Loder enters ch m Topsy. By Archie

R. Nevin “ . b g Schuyler Crosby. 12211

by Eclipse Dam by Printer....4 11

L. Dunham " g g Billy ............2 33332

G, A. Scott " b m Daisey Dean. By

Dolphus, dam American Boy ............... 34

T. Kate ch g Live Oak. By

H. Maher Eclipse, dam by Abdallah..5 " ch s Abdallah Chief, dam by Yankee Boy..6

Time, 2:38 $\frac{1}{2}, 2: 33 \frac{1}{4}, 2: 37,2: 36 \frac{1}{2}, 2: 39 \frac{3}{4}$.

JUNE 23D, 1869.

ASSOCIATION PARK, Detroit.

June 23rd.-Purse $\$ 400$. $\$ 250$ to first, $\$ 110$ to second $\$ 50$ to the third horse, for all horses owned in the State that have never beaten 2:50. Mile heats, best three in five, in harness. 


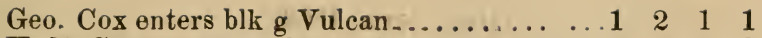

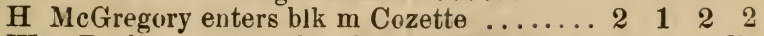
Wm. Darling “" b g Charley Welsh_..... 3 3 33 dis Time, 2:45, 2:45, 2:43ł, 2:44ł.

JUNE 23D, 1869. ASSOCIATION PARK, Detroit.

June 23rd.-Purse $\$ 900$. $\$ 550$ to first, $\$ 200$ to second, $\$ 150$ to the third horse, for all horses owned in the State, that have never beaten 2:30. Mile heats, be $t t^{\prime}$ :ree in five in harness.

John Demas enters ch m Idol................. 1

Peter Curran “ g g Meton.................. 332

W. C. Duncan “ blkg Victor Hugo... . . . .2 2

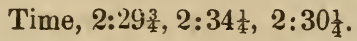

JUNE 23D, 1869.

LEAVENWORTH, Kansas.

JUNE 23rd.-Purse $\$ 300$. $\$ 200$ to first, $\$ 100$ to the second horse. Mile heats, best two in three.

T. R. Clark enters b m Kansas Maid.............. 1

E. G. Mann " s g Jack Morrow................2 2

H S. Forth “ b g Honest John..............

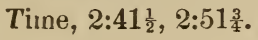

JUNE $24 \mathrm{TH}, 1869$.

\section{WATERTOWN PARK.}

JUNE 24tb.-Purse $\$ 250$. \$175 to first, $\$ 50$ to second, $\$ 25$ to the third horse, for horses that have never beaten 3:01.

A. M. Harris enters s g No Name. ........... 11

T. Ives $\quad$ " $\mathrm{g} g$ Gray $\mathrm{Jim} \ldots \ldots \ldots \ldots \ldots \ldots 2 \quad 2$

H. B. Griffen “" br g Browu Dick........... 3 3 3

T. G. Thomson " b s Gen’l. Sheridan.........dr

John Selleck " g m Nelly Gray...........dr

Time, 2:40, 2:38, 2:38. 


\section{6 \\ JUNE 24TH, 1869. \\ WATERTOWN PARK.}

JUNE 24th.-Purse $\$ 400$. $\$ 250$ to first, $\$ 100$ to second, $\$ 50$ to the third horse, for horses that have never beasen 2:40.

John Mott

H. T. Issam

$\begin{array}{ccccc}\text { enters br s Tete Matthews....1 } & 2 & 1 & 1 \\ \text { “" } \quad \text { ch m Nellie Issam......2 } & 1 & 2 & 2\end{array}$

A. M. Harris

L. L. Dorsey

Saml. Reynolds

R. Dalzell

" b g Frank Blair........3 dr.

“ b g Parks Golddust.....dr.

"6 b m Lady Lightfoot. ....dr.

" b s Phil Sheridan......dr.

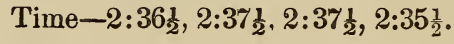

JUNE $24 \mathrm{TH}, 1869$.

\section{NARRAGANSETT PARK.}

JUNE 24 th.-Purse $\$ 1,500$, for horses that have never trotted better than 2:33. Mile heats, best three in five, in harness. $\$ 1,000$ to first, $\$ 350$ to second, $\$ 150$ to the third horse.

J. Donnovan enters $b \mathrm{~m}$ Lady Sheridan......... 11

H. S. Wright " blk m Jessie Wales.......... 2 2 2

H. A. Hall " blł $\mathrm{g}$ India Rubber Ben......2 $3 \quad 3$

Ben Mace “ b g Twang............... 4 4 4

Time-2:32, 2:283, 2:30.

JUNE 24TH, 1869.

NARRAGANSEIT PARK.

JUne 24th.-Purse $\$ 1,500$, for horses that never trotted better than 2:50. Mile heats, best three in five, in harness. $\$ 1,000$ to first, $\$ 350$ to second, $\$ 150$ to the third horse.

Dan Mace enters s g Locust.............. 1

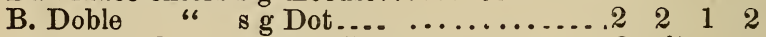

James Read " b g No Name ............. ...

Time-2:32, 2:37, 2:34, 2.30.

JUNE 24TH, 1869.

BUCKEYE COURSE.

Jene 24th.-Club Purse $\$ 200$, for green horses. Mile heats, best three in five, in harness.

C. Leggett enters blk g.'Tim, by Flying Cloud....... 1

H. Spillman " br g Charley, by Mohawk..........dis.

John T. Biggs " ch $m$ No Name................ dis,

Time-2:36. 
JUNE $24 \mathrm{TH}, 1869$.

\section{BUCKEYE COURSE.}

June 24th. - Club Purse, $\$ 200$, for horses that have never beaten 2:40. Mile heats, best three in five.

M. S. Forbes enters g g Gray Dick, alias Pilot, by Pilot, Jr......................2 \& 111

J. Miller enters ch $\mathrm{m}$ Favorite, by Abdallah, dam by Wagner .............................. 122

J. L. Fickland enters $\mathrm{r} g$ Aleck (late Joe Shawhan).......................

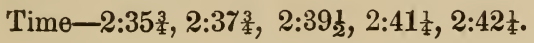

JUNE 24TH, 1869.

\section{ASSOCIATION PARK, Detroit.}

JuNe 24th.-Purse $\$ 450$. $\$ 125$ to second, $\$ 75$ to the third horse, for horses owned in the State that have never beaten 2:45. Mile beats, best three in five, in harness.

Geo. Cox enters blk g Vulcan................. 1

A. Rolf " g g Frank Rolf............... 2 2 2

H. Chappel " $\mathrm{g}$ s Bob Hunter................. 3 3 33

J.Demas " c in Phebe................dis.

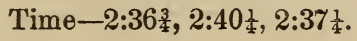

JUNE 24, I869.

INDIANAPOLIS, Ind

June 24th.-Purse $\$ 100$. Mile heats, best three in five, in harness.

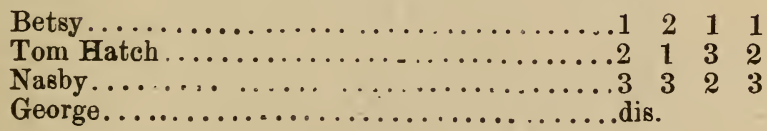

Time-2:57, 2:58, 3:03.

JUNE 25TH, 1869.

BUCKEYE COURSE.

JUNE 25th - Purse $\$ 200$, for horses that have never beaten 2:35. Mile heats, best three in five, in harness. 


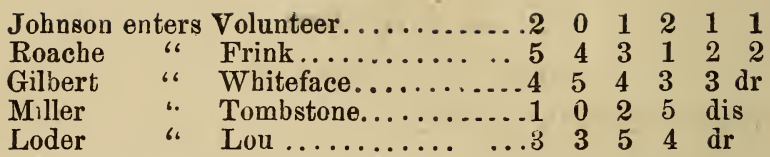

Time-2:31, 2:32 $\frac{3}{4}, 2: 34 \frac{1}{2}, 2: 35 \frac{1}{4}, 2: 36,2: 43 \frac{3}{4}$.

JUNE 25TH, 1869.

\section{WATERTOWN PARK.}

JUNe .25th.-Purse $\$ 1,000$. $\$ 700$ to first. $\$ 200$ to second, $\$ 100$ to the third hor sง, frue for all horses. Mile heats, best three in five, in harness.

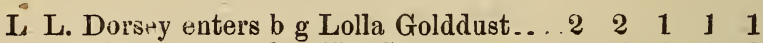

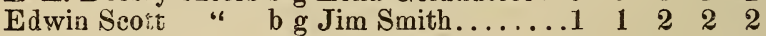

John Lovet " "blk g Gen. Butler.....dr

R. Dalz ll " br s Phil Sheridan dr

L. D. Doolitrle “" br g Dutch Boy...... dr

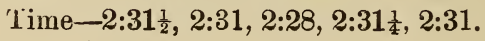

JUNE 25'ГI, 1869.

MAGNOLIA COURSE.

June 25th.-Purse $\$ 75$, free for all horses. Mile heats.

J. N. Shawhan enters $r$ g Dan Shawhan............ 1

C. B. Shawhan " br m Mary E Shawhan.......2 dis John Martn " s g Cowboy.............. dr

John McDonald " r m Norma.. ........... dr

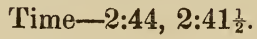

JUNE 25TH.

NARRAGANSETT PARK.

JUNE 25th.-Purse $\$ 1,500 . \$ 1,000$ to first, $\$ 350$ to second, $\$ 150$ to the third horse, for all horses that have never beaten 2:38. Mile heats, best three in five, in harness.

M. Roden enters b g Dresden..............

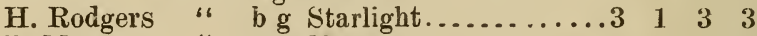

P. Mance " $\mathrm{g} g$ Mercer.............

H. Bradley " w g Stanley.............2 4 4 $4 \mathrm{dr}$

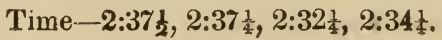




\section{JUNE 25TH. \\ BUCKEYE COURSE.}

JUNE 25th.-Club Purse $\$ 200$, for all horses that have never beaten 2:35. Mile heat, best three in five, in harness.

R. Johnson enters g g Volunteer, hy

Pilot, Jr., dam by Cripple

T. P. Roach enters $\nabla$ g Frank........

J. G Gilbert " ch o Whiteface, by

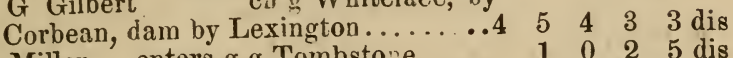

$\begin{array}{llllll}\text { J. Miller enters } \mathrm{g} g \text { Tombstone..... } & 1 & 0 & 2 & 5 & \text { dis }\end{array}$

J. B. Lowder " g $m$ Lew Loder

(Late Rush County Maid)........ $3 \begin{array}{lllll}3 & 3 & 5 & 4 & \mathrm{dr}\end{array}$

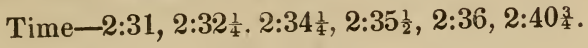

JUNE $26 \mathrm{TH}, 1869$.

\section{BUCKEYE COURSF.}

June 26th.-Consolation Stakes, $\$ 100$. Mile heats, best three in five, in harness.

J. Miller enters Favorite............... 2 1 1

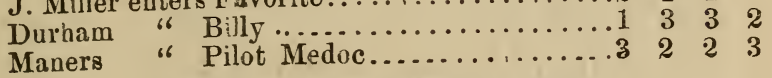

Time, 2:38, 2:39, $22: 38 \frac{1}{2}, 2: 37 \frac{1}{2}$.

JUNE 26TH.

NARRAGANSETT PARK.

JUNe 26th.-Purse $\$ 2,000$, for horses that never trotted better than 2.29. Mile heats, best three in five in harness. $\$ 1,300$ to first, $\$ 500$ to second, $\$ 200$ to the third horse.

W. H. Woodruff enters br s Mambrino Prince.9 11

N. W. Duryea “* $\quad$ g g Giay John.......

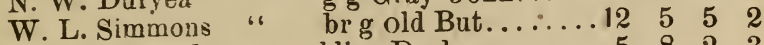

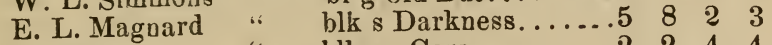

$\begin{array}{lllllll}\text { D.Pfifer " } & \text { blk m Cora. .......2 } & 2 & 4 & 4\end{array}$

H. Howe " " b g Lew Pettee.......

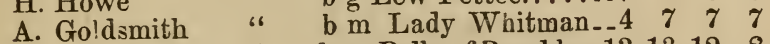

F. J. Nodine " " b m Belle of Brooklyn.13 $1312 \quad 8$

C. Chaplin " " 8 g W. H. Taylor....11 10119

M. Carroll “ b b g N. B. Palmer....6 111 1" 10

D. Mace “ “ $\quad$ b g Western New York.8 $6 \begin{array}{llll}6 & 3 & 11\end{array}$

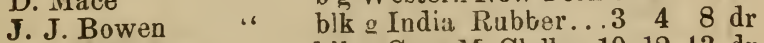

F. S. Stephens ": blkg Gen. McClellan.10 $12 \quad 13 \mathrm{dr}$

Time, $2.33,2.31 \frac{3}{4}, 2.32,2.33$. 
JUNE 26TH.

NARRAGANSETT PARK.

JUNe 26th.-Purse $\$ 3,000$ for all horses. Mile heats, best three in five, in harness. $\$ 2,000$ to first, $\$ 700$ to second, $\$ 300$ to the third horse.
M. Roden
D. Pfifer
B. Doble
N. Holcomb
C. Champlin
enters b m American Girl....... 1 1 1
"6 b m Lady Thorn ........4 4 4 2
“ $\quad$ b m Goldsmith Maid....3 33
.6 b m Lucy ............2 24
“ br g George Palmer.....5 55

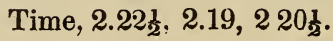

JUNE 26TH.

\section{BUCKEYE COURSE.}

JUNE 26th. - Club Purse $\$ 200$. Mile beats, best three in five, in harness.

T. P. Roach enters $\mathrm{g} g$ W. K. Thomas

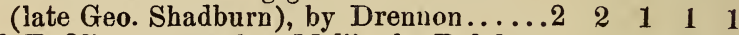

T. T. Oliver enters b m Mollie, by Dolphus,

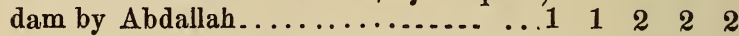

Time, 2.31 $\frac{1}{2}, 2.27 \frac{1}{2}, 2.29 .2 .30,2.31 \frac{1}{2}$.

JUNE $26 \mathrm{TH}$.

\section{INDIANAPOLIS COURSE.}

June 26th.-Handicap trot for a purse. Mile heats, best three in five.

$\begin{array}{lccccccc}\text { Moses } & \text { enters } & \text { b m Betsey (wagon)...3 } & 3 & 1 & 1 & 1 \\ \text { ('affee } & \text { " } & \text { c g Esaw (harness).... } & 4 & 4 & 2 & 2 \\ \text { Sain } & \text { " } & \text { g g TomHatch (wagon).4 } & 1 & 2 & 4 & \mathrm{dr} \\ \text { Hair } & \text { " } & \text { b g Canada dam (har.)..2 } & 2 & 3 & 3 & \mathrm{dr}\end{array}$

Time, $2.46 \frac{1}{2}, 2.54 \frac{1}{2}, 2.50 \frac{1}{2}, 2.52 .2 .52 \frac{3}{4}$.

JUNE 29TH.

FASHION COURSE, L. I.

JUNE 29th.-Sweepstakes $\$ 1,500$. Mile heats, best three in five, in harness.

$$
\begin{aligned}
& \text { D. Tallman enters b } m \text { Honey Dew ..... ...1 } \\
& \text { J. Lovett " br m Davenport neck mare.dis } \\
& \text { J. Murphy " br m Acorn...............dis } \\
& \text { Time, 2.41․․․ }
\end{aligned}
$$


JUNE 29TH.

\section{GOLDEN GATE PARK.}

JUNe 29th.-Purse and stake $\$ 300$. Mile heats, best three in five, in harness.

J L. Eoff enters br m Lady Dooley .......... 1

P. A. Finuegan " d g Pride of the Bay ......2 2

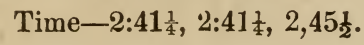

JUNE 29TH.

RENSSELAER PARK, Troy, N. Y.

JUNE 29th.-Purse $\$ 500$, for all horses that never trotted better than 3:00. $\$ 300$ to first, $\$ 125$ to second, $\$ 75$ to the third horse.

Wm. Borst enters s g Finnegan............. 1 1 $1122 \quad 1$

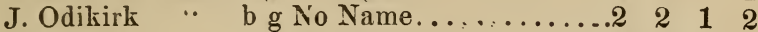

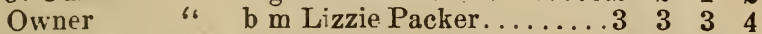

Dan Mace " sl g Locust...............

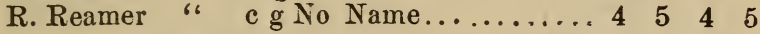

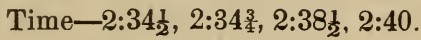

JUNE 29TH.

RENSSELAER PARK.

JuNE 29th.-Purse $\$ 900$, for all horses that have never trotted better than 2:37. $\$ 600$ to first, $\$ 200$ to second, $\$ 100$ to the third horse.

S. P. Dorsey enters s m Rosa Goiddust.......... 1 Owner

J. H. Phillips

D Akins

J. J. Hornbeck "

b m Topsey................. 2 2 2

ch $\mathrm{m}$ Nellie Isham... ......2 330

b s Ben Wood.............5 5

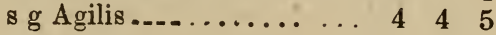

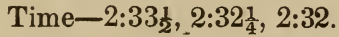

JUNE 29TH.

MYSTIC PARK.

JUNE 29th.-Match, $\$ 1,000$, best three in five, to harness.

J. J. Bowen enters ch g Good One............ 1 1 1

S. Hayes " ch g White Stocking.........2 2

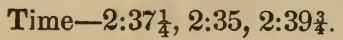


JUNE 30TH,

\section{GRAND RAPIDS.}

JUNE 30th.-Purse $\$ 150$, for horses owned in the State that have never beaten 3:00. Mile beats, in harness.

M. Bashaw erters ch m Flora............... 2

H. Ripple " br s Hiram Woodruff........2 12

J. H. Randall " b g Snip............... 3 3 3

W. B. Hewitt " b $\mathrm{m}$ Irish Maid...........4 dis

Time-2:55, 2:50, 2:52.

\section{JUNE 30TH. \\ RENSSELAER PARK.}

JUNE 30th. - Purse $\$ 800$, for all horses that have never trotted b tter than 2:44. $\quad \$ 500$ to first, $\$ 200$ to second, $\$ 100$ to the third horse.

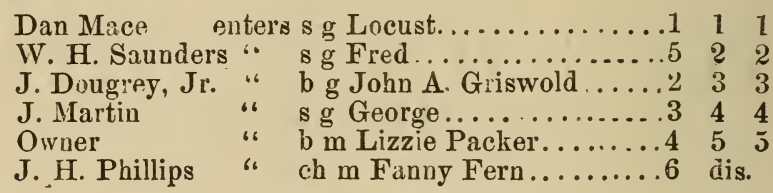

Time-2:52, 2:56, 2:51,

\section{JULY 1sT.}

\section{REN S̄SELAER PARK}

JuLy 1st.-Purse $\$ 1,200$, for all horses that have never trotted better than 2:28. $\$ 750$ to first, $\$ 300$ to second, $\$ 150$ to the third horse.

W. H. Saunders enters b $g$ Western New York...1 11

Dan Mace

Wm Borst

A Johnson

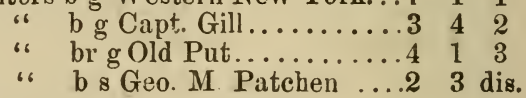

Time-2:33, 2:34눈, 2:32.

\section{JULY $1 \mathrm{sT}$.}

\section{RENSSELAER PARK.}

JULY 1st.-Purse $\$ 600$, for all horses that have never trotted better than 2:50. $\$ 350$ to first, $\$ 150$ to second, $\$ 100$ to the third horse. 


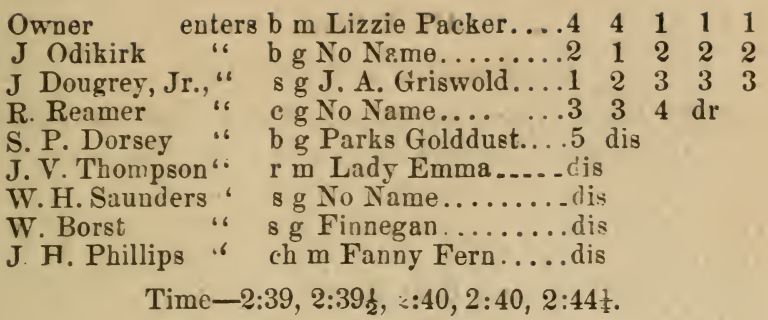

JULY 1s'T.

HAWKEYE COURSE, Clinton, Iowa.

JuLY 1st.-Purse $\$ 100$, for all horses that have never trotted better than 3:00. Mile heats, best three in five, in harness.

Richmond enters b $g$ No Name.............. 1

Owner "

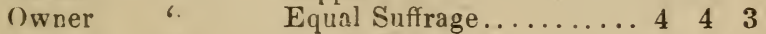

Owner " Black Bill............. $33_{3} 4$

Owrer ". Ristland Bny........... dis

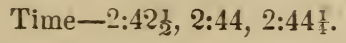

JULY 1sT.

GRAND RAPIDS.

JULY J st.-Purse \$275, for Michigan horses that have never beaten 2:50. Mile heats in barness. $\$ 200$ to first, $\$ 75$ to the second horse.

P. Curran enters g m Lady Clifford.............

J. Randall ". b g Snip..................

H. Rippell " b g Scalple..................

Time-2: $: 3,2: 50 \frac{1}{2}, 2: 55$.

JULY 1sT.

HAMILTON, C. W.

JULY 1st. - Purse \$150, for horses that have never beaten 3:00. $\$ 100$ to first, $\$ 50$ to the second horse. Mile heats, in harness

T. \& J Gillespie enters b m Lady Elgin.........2 211

P.:McSherry " b b htayner Boy.......... 2 2 2

W. Foster " " ch m Emily Foster ....... 33

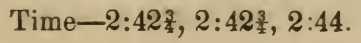




\section{* JULY 2D.}

\section{RENSSELLAER PARK.}

JULY 2d.-Purse $\$ 1,500$, for all horses that have never trotted better than 2.25. $\$ 900$ to first, $\$ 400$ to second, and $\$ 200$ to the third horse.

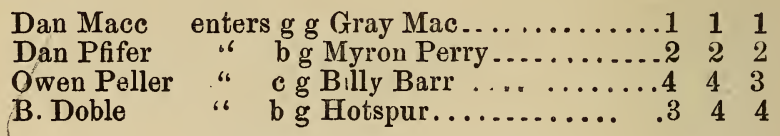

Time-2.291 $2.31,2.29 \frac{1}{2}$,

\section{JULY 2D.}

YPSILANTI, Mich.

JULY 2d.-Purse for horses that never have beaten 2.40. Mile heats, best three in five, in harness.

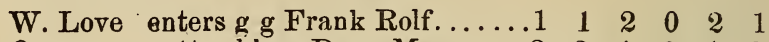

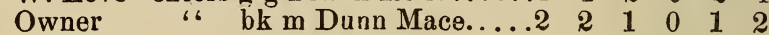

L. Carver “ g g Sea Foam .........3 3 3 dr

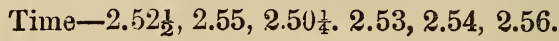

JULY 2D.

RITTERSVILLE, Pa.

JuLy 2d.-Purse $\$ 100$, open - to all horses. Mile heats, best three in five, in harness.

Frank Wagner enters s g Tormenter........... 1

D. S. Field “ b g Bill Jackson..........2 2

H. Ech

“ brg Monitor............dr

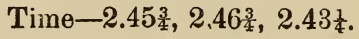

\section{JULY 2D.}

MYSTIC PARK, Mass.

JULY 2d.-Handicap race. Match $\$ 500$. Mile heats, best three in five.

D. H. Bukler enters br $m$ Fanchon (harness) .. $1 \begin{array}{llll}2 & 2 & 1 & 1\end{array}$

J. B. Jewell " b g Centreville (wagon)...2 1122

Time, 2:393 $2-37 \frac{1}{2}, 2: 38,2: 43 \frac{1}{4}$. 
JULY 2D.

\section{MYSTIC PARK.}

JuLy 2d.-Match $\$ 1,500$. Mile heats, best three in five, in harนess.

R. S. Wright enters ch \& Fearnaught, Jr ...... 11

J. E. Stewart "Sorrell stallion, by Green's

Hambletonian....................... 22

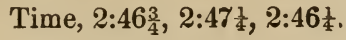

\section{JULY 2D.}

\section{GRAND RAPIDS.}

JuLY 2d.-Purse, free for all horses. Mile heats, best three in five, in harness.
O. A. Hickoch
enters s g Silas Rich....... $1 \begin{array}{cccc}1 & 1 & 2 & 1\end{array}$
J. Demas
" chm Idol..........2 2 12

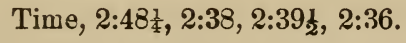

\section{JULY 3D.}

\section{CLINTON, Iowa.}

JULy 3d. - Purse $\$ 500$ for all horses. $\$ 300$ to first, $\$ 150$ to second, $\$ 50$ to third horse. Mile heats, best three in five, in harness.

$\begin{array}{lcccccc}\text { A. Gates } & \text { enters } & \text { Mace Eustice......2 } & \mathbf{1} & \mathbf{2} & \mathbf{1} & \mathbf{1} \\ \text { A. Carson } & \text { " } & \text { g m Queen of the West.1 } & 2 & \mathbf{1} & 2 & \mathbf{2} \\ \text { W. Vosburg } & \text { b g Chain Shot. ..... } & \mathbf{3} & \mathbf{3} & \mathbf{3} & \mathbf{3}\end{array}$

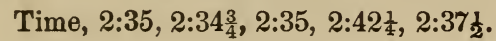

\section{JULY 3D.}

\section{UNIONVILLE, Orange Co. N. Y.}

JULY 3d.-Purse $\$ 50$ for horses that have never beaten 2:50. Mile beats, best three in five, in harness.

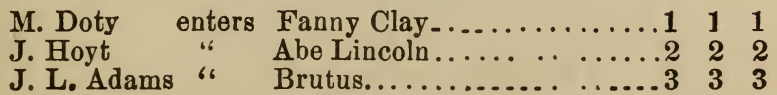

Time, 2:51, 3:03, 3:01.

\section{DOTY'S PARK Poughkeepsie.}

JoLY 3d.-Purse $\$ 500$ for horses that have never beaten 2:36. Mile heats, best three in five, in harness. 
A. Johnson

J. Hornbec

enters b g Geo. M. Patchen, Jr....1 11 Dr. Ogle

" s g Agellis..............2 3 2

" s g Pet................

Tine. 2:35. 2:44, 2:43

JULY 3D.

OMAHA PARK.

JuLY 3d.-Match $\$ 50$. Mile heats, best three in five, in harness.

Wm. liodges enters Johnny Come Up........ 111

M. C. Wilbur " br m Lady Hamilton......2 2 2

Time, 3:00, 2:51, 2:53.

JULY 3D.

SPRINGFIELD, Mass.

JULY 3l. $\neg$ Purse $\$ 100$ for horses owned in the county. Mile heats, best three in five, in barness.

\begin{tabular}{lcccccc} 
Edward Allen & enters blk m Black Betty..5 & 5 & 1 & 1 & 1 \\
Reed & “ & b m Rosa........... & 1 & 2 & 5 & 5 \\
Davis & “ & 8 g Coffe Grinder...2 & 3 & 4 & 3 & 2 \\
E. C. Robinson & “ & b m Highland Maid.4 & 2 & 3 & 2 & 3 \\
\hline
\end{tabular}

Time, 2:54, 2:54, 2:53, 2:51, 2:51.

JULY 3D.

SPRINGFIELD, MasS.

JuLy 3d.-Purse $\$ 175$ for all horses. Mile heats, best three in five, in harness.
G. Kingsbury
enters b
b g Emperor........... 1 1 1
W. Elliot
g g Spot.............2 $2 \quad 2$

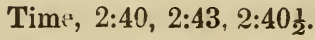

JULY 3D.

\section{DANBURY PLEASURE PARK Ccnn}

JuLy 3d.-Purse $\$ 175$ for all horses. Mile heats, best three in five, in harness.

C. Dickennan

R. Mabbit

8. Sniffen

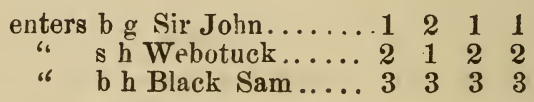

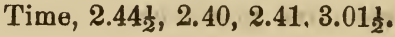


JULY 5TH.

MINNEAPOLIS, Ind.

JuLy 5th.-Purse \$125. \$25 to second. Mile heats, best three in five, in harness.

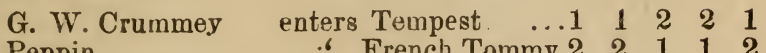

Peppin

$\begin{array}{llllll}6 & \text { French Tommy.2 } & 2 & 1 & 1 & 2\end{array}$

Time, 2.41, 2.42, 2.43, $2.45 \frac{1}{2}, 2.47 \frac{1}{2}$.

JULY 5 TH.

SOUTH WEYMOUTE, Mass.

JuLY 5th.-Purse $\$ 100$. Mile heats, best three in five, in harness

(*. B. Blanchard enters b m Kitty Blanchard..1 11

Alden Nash

" $8 \mathrm{~g}$ Weymouth Boy....2 22

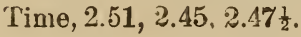

JULY ร̆тн.

DETROIT, Mich.

JuLY 5th. - Purse $\$ 2,000$ for all horses that have never beaten

225. Mile heats, best three in five, in harness.

M. Calvin enters b s Pilot Templo....... 1 1 12

J. Demas “ " ch m Idol. ............ 4 4 1

O. A. Hitckock " $\quad$ s g Silas Rich........... $322 \quad 3 \quad 2$

W. H. Sullivan“" gs Gray Eagle..........

Time, 2.30, $228 \frac{1}{4}, 2.30,3.30 \frac{1}{2}$.

JULY 5 TH.

DETROIT, Mich.

JULY 5th.-Purse $\$ 500$ for borses owned in the State, that have never beaten 2.40. Mile heats, best three in five, in harness.

Geo. Cox enters blk g Vulcan.............. 1

A Rolfy " $\mathrm{g} g$ rrank Rolfe.............2 2

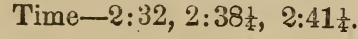

\section{JULY 5TH}

\section{UTICA.}

JULY 5th.-Match. Mile heats, best three in five, in harness.

Owuer enters Schuyler Boy ............

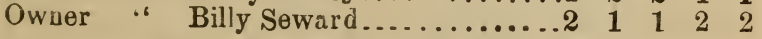

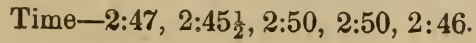




\section{JULY 5TH.}

\section{WORCESTER PARK, Mass.}

JULY 5th.-Purse $\$ 100$, fur horses owned in the County that never beat 2:50. Milu heats, best three in five, in haruess.

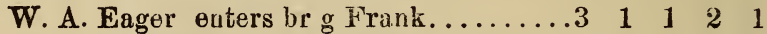

W. M. Mandell “ b in Trifle....,......

$\begin{array}{llllllll}\text { T. F. Lyon } \quad \text { " } & \text { g m Lady Lyon. } & \ldots . .2 & 2 & 2 & 1 & 2\end{array}$

C M. Howland " b g Target . .

Time-2:51, 2:52⿺ 2: 2:48, 2:49, 2:52.

JULY 5TH.

WORCESTER PARK, MasS.

JULY 5th.-Purse $\$ 300$, for all horses that have never beaten 2:39. Mile heats, best three in five, in harness.

M. Carroll enters b g N. B. Palmer............ 1

E. L. Norcross " blk m Jessie Wales.........2 22

Time-2:37, 2:37, 2:35.

\section{JULY 5 TH.}

\section{SYRACUSE DRIVING PARK.}

JULY 5th.-Purse \$125. Mile heats, best three in five, in harness.

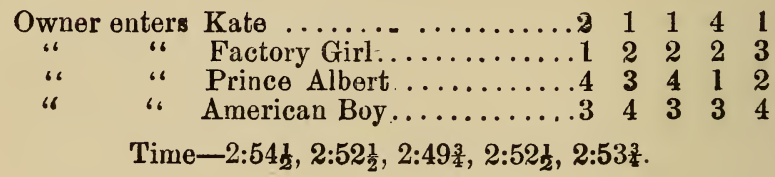

\section{JULY 5TH}

DOTY'S PARK, Poughkeepsie.

JuLY 5th.-Purse $\$ 300$. Mile heats, best three in five, in harness.

- Dr. Ogle enters s g Pet................... 1

M. Roden " b g Jim Libby................2 2

Time-2:43, 2:40, 2:37. 


\section{JULY 5 TH. DOTY'S PARK.}

JULY 5th.-Purse $\$ 500$, for all horses that have never beate 2:40. Mile heats, best three in five, in harness.

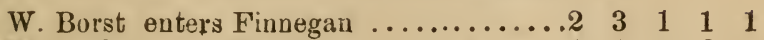

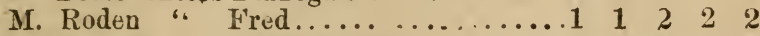

$\begin{array}{lllllll}\text { J. Dugrey “ Black Knight.... } & \ldots \ldots & \ldots & 2 & 4 & 3 & 3\end{array}$

J. Malone " Young Wilkes.........4 42 dis.

Time-2:35, 2:38, 2:38, 2:37, 2:39.

JULY 5 TH.

DOTY'S PARK.

July 5th.-Purse $\$ 1,000$, for horsss that have never beaten 2:30. Mile heats, best threg in five, in harness.

M. Dorsey eaters Rosa G.lddust $\ldots \ldots \ldots \ldots \ldots 1 \quad 1 \quad 1$

A. Johnson “" G. M. Patchen, Jr....... 522

D. Mace “ Capt. Gill .............2 33

W. Borst “ Old Put.................... 44

S. G. Bailey " Topsey................ 55

Time-2:32, 2:32, 2:32:

JULY 5 TH.

BUCKEYE COURSE.

JULY 5th. - Sweepstakes \$50. Entrance P. P. Mile heats, best three in five, in harness.

Benninger enters $\mathrm{b} g$ Lexington $\ldots \ldots \ldots \ldots \ldots 1 \quad 1 \quad 1$

Reynolds " b g Day. ................... 2

Shoenberger " b g Harkaway................ dis

Time-2:48, 2:491, 2:463.

JULY 5 тн.

MANCHESTER, N. H.

July 5th.-Purse \$150. Mile heats, best three in five, in harness.

Keyes $\quad$ enters b m Mountain Girl.... 1

Putney \& Hopkins " gr m Mossenger Girl...2 22

Time-2:49, 2:41. 
JULY 5TH.

GOSHEN, Orange Co., N. Y.

JULY 5Th.-Purse $\$ 50$, for all horses that have never beaten 2:50. Mile heate, best three in five, in harress.

L. Hoyt

H. C. Bishop

2द्र M. Doty

C. Millspaugh

T F. Swith

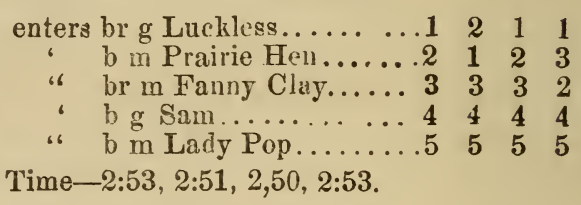

JULY 5TH.

COSREN, N. $Y$.

JuLy 5TH.-Match $\$ 50$. Mile heats, in harness.

H. C. Bishop enters b m Prairie Hen........ 1 o 1 J. Hoyt ." br g Luckless .............

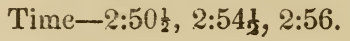

\section{JULY 5TH.}

\section{IEAVENWORTH, Kansas.}

JuLY 5TH.-Purse, for all horses that never trotted for money (fairs excepted). Mile hoats.

H. S. Foote enters b $g$ Henest John........ 3 cis

D. Wasts ". b n. Kate Wasson........ 4 dis

G. Marn

L. Sargent

" r g Frank Mann............ 1 2

. $\mathrm{r}$ g Ned..............2 11

Tine-2:463, 2:42, 2:42.

JULY 5 TH.

IEA VENWORTH, Kansas.

JuLy 5tb.-Citizens' Purse $\$ 600$. Mile beats.

T. Best enters g in Tackey .............

T. Paimer " s g Blind Billy.............2 1

Time-2:38 $\frac{1}{2} \cdot 2: 38,2: 36$.

\section{JULY 6TH.}

\section{ELLICOTTSVILLE.}

JULy 6th.-Purse $\$ 350$, for all horses that have never beaten 2:32. Wile heats, best three in five, in harness. 
T. Miles enters b m Belle.............. 1 1 11

S. H. Titus " g g Whitebird.......... 3 2 2

F. Thompson “ b g Frank............. 3 dis

Time-2:42ł, 2:33, 2:38, 2:39.

\section{JULY 6TH.}

KNOX TROTTING PARK. Rockland, Me.

JULY 6th.-Purse \$150. Mile heats, best three in five, in harness.

G. M. Robinson enters Colonel Lakeman.......1 11

Owner

Owner

6. Carlotta................ 2 2 2

.. Lady Chapman...........3 $3 \quad 3$

Tine 2:35, 2:39, 2:44.

JULY ซTH.

BURLINGTON, $\nabla t$.

JuLy 7th.-Match \$400. Mile heats, best three in fire, in harness.

Owner enters Col. Kilpatrick ............... 111

Owner " Sorrell Dan................2 2

Time-2:44, 2:45, 2:42.

JULY 7 TH.

MYSTIC PARK, Mass.

JULY 7th.Match $\$ 1,500$. Mile heats, best three in fire, to wagons.

B. S. Wright enters ch s Fearnaught, Jr...... 1 1

J. E. Stewart " Sorrell Stallion, by Grees

Hambletonian..................... $2 \quad 2$

Time-2:493, 2:47, 2:45.

\section{JULY 7TH. \\ MYSTIC PARK.}

July 7th - Match $\$ 2,000$. Mile heats, best three in five, to wagons.

M. Roden enters b g Myron Perry............ 11

B. Doble " b g Hotspur ..............2 2 22

Time-2:27, 2:3), 291. 


\section{JULY 7TH. \\ NASHVILLE, Tenn.}

JuLy 7th.-Purse $\$ 75$, for horses that have never beaten $3: 20$ Mile heats, best three in five, in harness.

W. S. Widner enters $\mathrm{r}$ g Ku Klux..............
G. Futcher

Time-2:54, 2:55, -

\section{JULY 7TH.}

\section{ELLICOTTSVILLE.}

JULY 7th. - Purse $\$ 150$, for horses that have never beaten 2:50. Mile heats, best three in five, in harness.

D. O. Wilson enters b m Kitty Gale...2 $3 \begin{array}{lllll}3 & 2 & 1 & 1 & 1\end{array}$

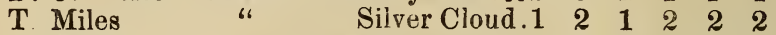

S. Mattoon “" b g Capt. Jinks ... 3 1 13 dis.

C. Frank “ b $\mathrm{m}$ Fearless..... dr.

D. E Bartlett " br g Brown Billy. dr.

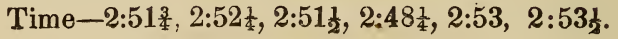

क

JULY 7TH.

ELLICOTTSVILLE.

Joly 7th.--Purse $\$ 300$. Mile heats, best three in five, in harness.

A. Lewis enters b m Clara.................. 11

W. Brown "6 br g Dic - Hilliard............ 2 2 2

S. H. Titus " White Bird............... 33

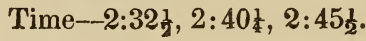

JULY 8TH.

MYSTIC PARK.

JuLy 8th.-Match $\$ 2,000$. Mile heats, best three in five, in harness.

B. Doble enters b m Goldsmith Maid.......... 1 1 1

J. J. Bowen “" b m Lucy................2 22

. Time- $-2: 20 \frac{1}{2}, 2: 21_{\frac{1}{2}}, 2: 21 \frac{1}{4}$. 
JULY 8TH, 1869.

\section{UNION COURSE, L. I.}

July 8th.-Match $\$ 1,000$. Play or pay. Mile heats, best three in five, in barness.

W. McMann enters w $g$ White Horse ........ 1112

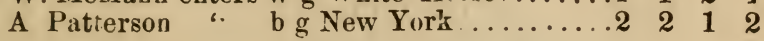

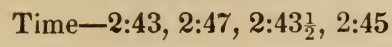

JULY 8TH, 1869.

NASHVILLE, Tenn.

JuLy 8th.-Purse \$125, free for all horses. Mile heats, best three in five, in harness.

C. A. Wand enters Little George............. 1

—. Owens " Geo. Spaulding.............

S. Widener " Ku Klux................ dis.

Time-2:463, 2:40, 2:401.

JULY 9TH: 1869.

NASHVILLE, Tenn.

JoLY 9th.-Purse \$125. Mile heats, free for all horses.

S. Wid. ner enters $\mathrm{Ku}$ Klux.............. 2 1 1

C. A. Wand ". Eliza Jane................

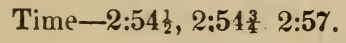

JULY 10TH, 1869.

HARTFORD, Conn.

JoLY 10th.-Purse \$75, for horses that have uever beaten 2:50 Mile heats, best three in five, in harness.

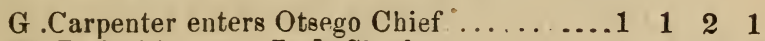

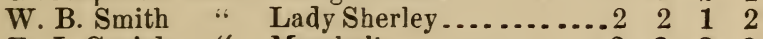

E. J. Cusick " Marshall..............

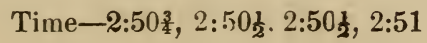

JULY 10TH. 1869.

FASHION COURSE, I. I.

JULY 10th - Match $\$ 500$. Mile heats, best three in five, in harness. 
J. Cudney enters b $g$ Twist...............1 1 is M. Roden " b g Young Commodore.........2 2 Time-2:44a, 2.46, 2:51.

JULY $12 \mathrm{TH}, 1869$.

FASHION COURSE, I. I.

JULY 12th._Purse $\$ 2,500$. Mile heats, best three in five, in harness.

M. Roden enters b m American Girl.........1 $1212 \quad$ I

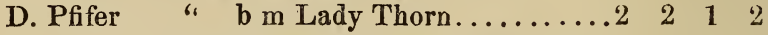

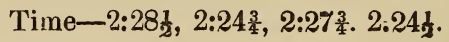

JULY $13 \mathrm{TH}, 1869$.

RENSSELAER PARK.

JuLY 13th-Purse $\$ 1,250$. Mile heats, best three in five, in harness.

D. B. Bissell enters $\mathrm{c} g$ Billy Barr........... 1 1 1

C. S. Green " b g Rolla Golddust.........2 2

Time-2:321 2: 2:31, 2:33.

JULY $15 \mathrm{TH}, 1869$.

BUFFALO DRIVING PARK.

JULY 15th.-Purse $\$ 300$ gold. Mile heatB, best three in five, in harness.

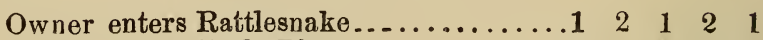

Owner " Lady Blance..............2 $12 \begin{array}{llll}1 & 2 & 1 & 2\end{array}$

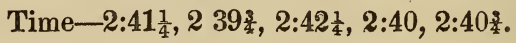

JULY 15TH, 1869.

UNION COURSE, I. I.

JULY 15th.-Sweepstakes $\$ 750$. Mile heats, in harness.

W. Nelson enters ch $\mathrm{m}$ Mollie Gulddust (wagon).1 1

H. Howe " g g Mazeppa (barness)........ 32

J. V. Thompson “ b m Lady Hurd (barness) .....2 23

Time-2:581, 2:54. 
JULY 15TH, 1869.

\section{GREAT FALLS (N. H.) RIDING PARK.}

JULY 15th.-Purse $\$ 150$, for horses that have never beaten 3:00. Mile heats, best threo in five, iu harness.
S. Hayes
H Woodruff
F. Swain
J. Churchill
H. H. Sinith
J. Mooney
enter's s m Lady Rollins.........3 11 1 1

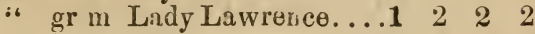
" b g Reuben............ 5 4 43 3
“ g g Wild Eddy........6 $3 \quad 3 \quad 4$ dis
" r m Belle Gibbs........2 dis
" b m Sally...........4 dis

Time-2:45, 2:45, 2:44, 2:47.

JULY 15TH, 1869.

\section{GREAT FALLS (N. H.) RIDING PARX.}

JULY 15TH.-Purse \$225, for horses that bave never beaten 2.40. Mile heats, best three in five, in harness.

H. Bradley enters blk g Black Joe ............ 1

S. Hayes " ch m Fanny ............... 2

J. Mooney “ $\quad$ b m Sally ...................

S. Jacobs " $\quad$ r s Butcher Boy.... . .

W. H. A. Hott “ $\quad$ rm Rosa................ 5

G. Nutter "6 b g Martin Luther........... dis

F. Jones "Mountain Maid............ dis

M. Eldridge ‘ Belle of Portsmouth.......... dis

Time, 2:40, 2:40, 2:40.

\section{JULY 16TH.}

\section{GREAT FALLS, (N. H.) RIDING PARK.}

JULY 16th.-Purse, $\$ 175$ for horses that have never beaten 2:50. Mile heats, best three in five, in barness.

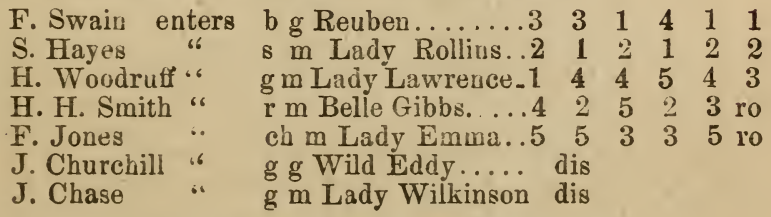

Time, 2:48, 2:43, 2:46, 2:46, 2:45, 2:49. 


\section{6 \\ JULY 16TH, 1869.}

GREAT FALLS, N. H. RIDING PARK.

JULY 16th - Purse $\$ 350$, for horses that have never beaten 2:30. Mile heats, best three in five, in harness.

A. W. Worcester enters blk g Gen. McClellan4 $11 \quad 1 \quad 1$

H. Woodruff

L. M. Ray

J Moeney

R. S. Wright

W. Locke

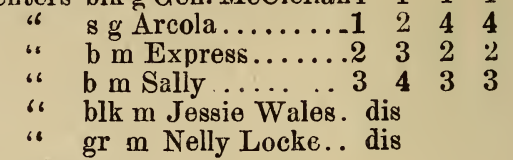

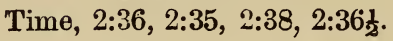

JULY 16TH, 1869.

FASHION COURSE, I. I.

JuLY 16th.-Match $\$ 1,000$. Mile heats, best three in five, in harness.

$\begin{array}{lcllll}\text { B. Dobble } & \text { enters } & \text { b m Goldsmith Maid....1 } & \mathbf{1} & \mathbf{1} \\ \text { C. Champlin } & \text { " } & \text { b g George Palmer.....2 } & 2 & \mathbf{2}\end{array}$

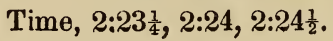

JULY 16TH.

FASHION COURSE, I. I.

JULY 16th.-Match $\$ 50$. Mile heats, best three in five to wagons.

J. Murphy

M. Roden

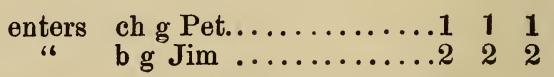

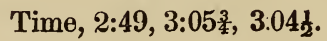

\section{JULY 17TH, 1869.}

EASTON, Pa.

JULY 17th.-Purse and stake $\$ 100$. Mile heats, best three in five.

Joseph Fisher enters blk m Flora Morrill (harness). $1 \quad 1 \quad 1$ T. Newman " s s Dr. Jackson (wagon)..2 2

W. H. Dawes “ s m Miller's Damsel (wagon)3 $3 \mathrm{dr}$

Time, 3:15, 3:03, 2:59. 
JULY 17 TH.

FORD'S PARK, Dэnver, Col.

JULy 17th.-Purse $\$ 150$. Milo heats, in harness.

J. M. Broadwell enters blk m Bet............ 1

J. M. Harris " $\quad$ s g Johu..............2 2

A. A. Cook $\quad$ " b m Kate.............. dr

Time, $2: 57,2: 56 \frac{1}{2}$.

JULY 17TH, 1869.

WORCESTER COURSE, Mass.

JULY 17th.-Purse $\$ 00$. Mile heats, best three in five, in harness.

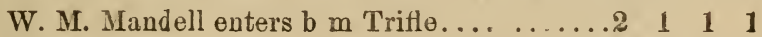

T. F. Lyon " " $\mathrm{g} m$ Lady Lyon.........

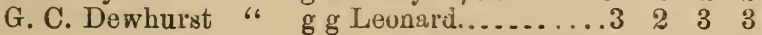

Time-2:47, 2:46, 2:47 $\frac{1}{2} .2: 45$,

JULY $17 \mathrm{TH}, 1869$.

UNION COURSE, I. I.

JULY 17th.-Match \$200. Milo heats, best three in five, to wagons.

J. Murphy enters b g Red Rover........... 1 1 2

M. Roden " b g Young Commodore......2 2 J 2

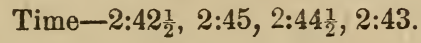

JULY $17 \mathrm{TH}, 1 \mathrm{~s} 69$.

UNION COURSE I. .

Jur.Y 17th-Match \$:, 600 . Mile heats, in haruess.

M. Roden enters b m American Girl...........1 1

S. McLaughlin " b g Mountain Boy...........2 *2

Time-2:233, 2:213.

JULY 17TH, 1869.

\section{DEXTER PARK, Chicago.}

JuLy 17th.-Match $\$ 1,000$, Milo heats, best three in five, in harness.

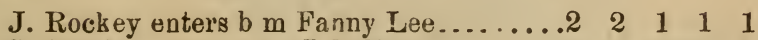

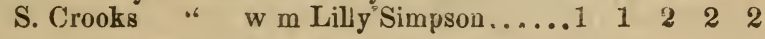

Time-2:39, 2:37 $\frac{3}{4}, 2: 33 \frac{3}{4}, 2: 40 \frac{1}{4}, 2: 40$. 
JULY $17 \mathrm{TH}, 1869$.

\section{DEXTER PARK, Chicago.}

JULY 17th.-Purse $\$ 400$. Mile beats, best three in five, in harness.

H. H. Yates enters b m Kate Hazard.......... 1

M. Tundall " b g Bay Jim.............2 2

J. L'redmore " 8 g John Tonley...........

Time-2:393 $2: 38,2: 40$.

JULY 17TH, 1869.

BUTMERFIELD COURSE, Utica.

JULY 17th.-Match $\$ 500$. Mile heats, best three in five, in harness.

B. Golden enters $\mathrm{b} g$ Billy $\ldots \ldots \ldots \ldots \ldots \ldots \ldots$ 1 1

H. Wilson " $\mathrm{g} g$ hockingham ............2 2

Time-2:35, 2:36, 2:331.

JULY $19 \mathrm{TH}, 1869$.

FASHION COURSE, I. I.

Jucy 19th.-Purse $\$ 400$. Mile heats, lest three in five, in harness.

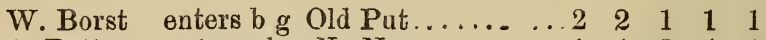

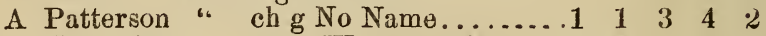

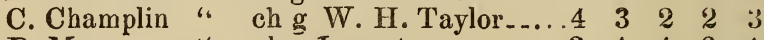

D. Mace “ ch g Locust............ 3 4 $4 \begin{array}{cccc}4 & 4 & 4\end{array}$

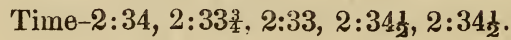

JULY 19TH, 1869.

DAYTON, 0.

JuLy 19ih. - Purse $\$ 50$. Mile heats, to go as you please.

C. C. Cathcart enters $g$ g Harry Clay .............. 1

— Greenleaf " b g Velocipede.............2 2

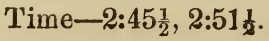

JULY 20TH, 1869.

\section{SUFFOLK PARK.}

JuLY 20th.-Purse $\$ 1, C 00$. Mile heats, best three in five, to wagons. 
M. Roden enters b g Myron Perry............. 1

R. Doble “ b b g Hotspur................2 2

Time-2:31, 2:29, 2:33.

JULY 20TH, 1869.

BUFFALO DRIVING PARK.

JoLY 20th.-Purse $\$ 500$. Mile heats, best three in five, in harness.

R. Johnson enters $g \mathrm{~g}$ Volunteer............. 1

W. Brown " b m German Gir]............ 203

A W.Dimmick “ s g Bloomer.... .........2 dr.

Time-2.35, 2.54, 2.54 .

JULY 20TH, 1869.

MYSTIC PARK.

JuLY 20th.-Match $\$ 1,5$ C0. Mile heats, best three in five, in harness.

D. Mace enters ch s Fearnaught, Jr........1 11

J. E. Stewart “ 8 s by Green's Hambletonian.2 22

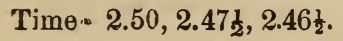

JULY 20TH, 1869.

MYSTIC PARK.

JULY 20th.-Sweepstakes $\$ 250$. Mile heats, best three in five.

D. Bigley enters Roan Mare (in haruess)......2 122 I 1

J Beckler " 8 g Sorreil John (in harness)..1 121222

H. Bradley " b m Jennie Spencer(to wagon) 3333 dr.

Time-2.46 $\frac{3}{4}, 2.43 \frac{1}{2}, 2.42 \frac{1}{4}, 2.42 \frac{1}{2}, 2.45$.

JULY 21st, 1869.

SUFFOLIK PARK.

JuLY 21st.-Match \$2,000. Mile heats, best three in five, in harness.

B. Doble enters b m Goldsmith Maid........... 11

M. Roden " b m American Girl..............2 2

Timo-2.22, 2.23, 2.30. 
JULY 21st, 1869.

GOLDEN GATE PARK, San Francisco, Cal.

JULY 21st.-Purse and stake \$250. Mile heats, best three in five.

J. L. Eoff enters g s Capt. Hawford.........1 113221

E. Lusk " $\quad \mathrm{g}$ s Dick Gough (to wagon). 3212112

- Kennedy “ 8 g Arthur Granger........... $4 \begin{array}{llll}3 & 4 & 3 & 3\end{array}$

Roe Allen

Timu-2.43, 2.381 $2.40,2.39,2.39$.

JULY 24TH, 1869.

FORD'S PARK, Denver, Col.

JoLy 24th.-Purse \$150. Mile heats, in harness.

R. Hammell enters blk g Ghost.................. 1

C. A. Cook " b m Kate.................... 2

J. Collins " blk m Belle Brandon............3 dr.

Time-3.00, 2.55.

JULY 24TH, 1869.

FASHION COURSE, L. I.

JULY 24th. - Match $\$ 500$. Mile heats, best three in five.

J. Murphy enters ch m Louise (to wagon)........1 1121

A. Whitson " blk g Little Frank (in harness) 2212

Time-2.44, 2.37, 2.433, 2.40

JULY 28TH, 1869.

BINGHAMPTON, N. Y.

JULY 28th.-Purse $\$ 150$, for horses that have never beaten 2.30. Mile heats, best three in five, in harness.

J. A. Bell

J. H. Bertholf

H. A. Hollis

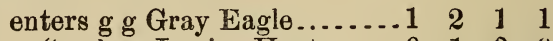

" b in Louisa Hoyt....... 0 1 000

" b m Mary Barber....... 0 o 00

W. Van Benscoten “ b g John............. 0 O 0000

Time, 2.38, 2.39, 2.40, 2.43.

JULY 28TH, 1869.

BINGHAMPTON, $\mathbf{N}$. $\mathbf{Y}$.

JuLy 28th.-Purse $\$ 300$. Mile heats, best three in five, in harness. 
J. H. Berthelf enters b s Major Edsall........ 1 1 0011

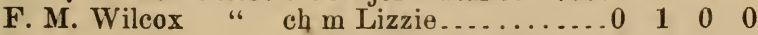

Owner

" - g George Smith.. .... 00000

Time, 2.40, $236 \frac{3}{4}, 2.35 \frac{1}{2}, 2.34$.

JULY 28TH, 1869.

OMAHA TROTIING PARK.

JULY 28th.-Match $\$ 500$. Mile heats, best three in five, in harness.

O. Holstein enters blk g Cul. Jennison........ 1 1 12

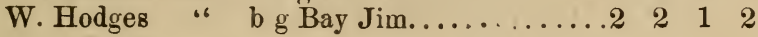

Time-2.55 $\frac{1}{2}, 2.42 \frac{1}{2}, 2.46 \frac{3}{4}, 2.46$.

\section{JULY $28 \mathrm{TH}, 1859$. \\ UNION COURSE, I. I.}

JuLY 28th.-Match $\$ 2,000$. Mile heats, best three in five, in haruess.

D. Pfifer enters b m Lady Thorn............. 11

B. Doble " b m Goldsmith Maid..........2 22

Time-2.21 $\frac{3}{4}, 2.20 \frac{1}{2}, 2.21 \frac{1}{4}$.

JULY 28TH, 1869.

\section{UNION COURSE, I. I.}

JULY 28th.-Match $\$ 500$. Mile heats, best three in five, to wagons.

J. Murphy enters b g Red Rover............ 1

B. Daniels “ b g Commodore.............2 22

Time, $2.50 \frac{1}{2}, 249,2.51$.

JULY 28TH, 1869.

URBANA, III.

JuLy 28th.-Purse \$175, free for all. Mile heats, best three in five, in haruess.

M. Colvin enters blk m Fanny Kemble....... 1

J.H Hight " b g Strideaway...........2 2

F. M. Burrows “ b in Ida............. 3 3 3

Time, 2.42논, 2.38, $2.36 \frac{1}{2}$. 
JULY 28TH, 1869.

URBANA, $\mathrm{II}$.

JuLY -28ta. - Purse $\$ 100$. Mile heats, best three in five, in harness.

W. Moure enters d $\mathrm{m}-\ldots \ldots \ldots \ldots \ldots 1 \quad 1 \quad 1$

J. H. H ght " b s

F. M. Burrows " Champion Black Hawk .3 dr

Time, 2.42⿺ 2.423, 2.41.

JULY $29 \mathrm{TH}, 1869$.

BINGHAMPTON, N. Y.

JuLY 29th.-Purse $\$ 200$. Mile heats, best three in five, in harness.

J. A. Bell enters $g$ g Gray Eagle......... 1 1 1001

Owner " b g Geo. Smith..........

J. H. Bertholf “ b m Louisa Hoyt......... 00000

H. A. Hollis " b b mary Barber........ 0 0 000 0

Time, $2.37 \frac{3}{4}, 2.38 \frac{1}{4}, 2.39 \frac{1}{2}, 2.37 \frac{1}{2}$.

JULY 29TH, 1869.

BINGHAMPTON, N. $\mathbf{Y}$.

JuLY 29th.-Purse $\$ 1,200$. Free for all horses. Mile heats, best three in five, in harness.

A. Lewis enters b m Clara (for Crazy Jane) $2 \begin{array}{llll}2 & 1 & 1 & 1\end{array}$

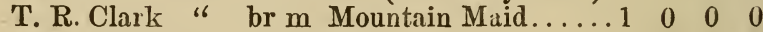

J. M. Pettit “ b m Fanny Allen.......... 0 0 0000

Beebe " g g John................

Time, $2.30 \frac{1}{2}, 2.31 \frac{1}{4}, 2.34 \frac{1}{4}, 2.32 \frac{1}{2}$.

JULY 29TH, 1869.

RIVERSIDE PARK, Mass.

July 29th.-Gentlemen's Race. Purse $\$ 200$. Mi e heats, best three in five, in harness. Owners to drive.

Owner enters g m Fanuy................. I 11

Owner " b m Kate..................... 2 2

Owner " g mEva....................

Owner " brg Poison..................... 4 4 4

Time, 2.521 $2.44,2.43$. 
JULY 29TH, 1869.

\section{SUFFOLK PARK COURSE.}

JoLY 29th.-Match $\$ 1,000$. Mile heats, best three in fire, in harness.

J. Levitt enters $g$ s Ironsides............... 1 1

B. Dobble “ b g Dot.................. 2 2

Time, $2.34,2.34 \frac{1}{2}, 2.36$.

JULY 29TH, 1869.

BANGOR, Me.

JuLY 29th.-Purse \$200.Mile heats, best three in five, in harness.

Mr. Palmer enters b g Bully Brooks.......... 1

Mr. Garland " b m Lady................ 2

Time, 2.47, 2.49, 2.54 .

JULY 29TH, 1869.

UNION COURSE, I. I.

JULY 29th.-Match $\$ 2,000$. Mile heats, best thres in five, in harness.

R. Fletcher enters $\nabla \mathrm{g}$ Robin............. 11

D. Pfifer " ch g Americus............2 \& 2

Time, $2.41 \frac{1}{2}, 2.42,2.42 \frac{1}{2}$.

JULY 29TH, 1869

SYRACUSE, N. Y.

JULy 29th.-Match $\$ 100$. Mile heats, best three in flve, in harness.

Owner enters Canada Boy............. 1121

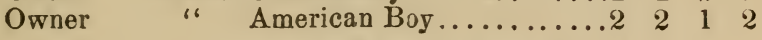

Time, 2.53, 2.54, 2.55⿺ 2.53 .

JULY 30TH, 1869.

MYSTIC PARK, Mass.

JuLy 30th.-Match $\$ 200$. Mile heats, best three in fire, in harness.

C. M. Beckler enters \& g Sorrell John........ 1

J. Stewart " b m Jennie Spencer.....2 22

Time, 2.43눈, 2.44t, $248 \frac{1}{2}$. 


\section{JULY 30TH, 1869. MYSTIC PARK, Mass.}

JuLY 3 3 th. - Sweepstakes $\$ 490$. Mile heats, best three in five. J. J. Bowen enters b g Clifton (for Jack

$\begin{array}{lllllr} & & \text { Lewis, to wagon)..2 } & 1 & 1 & 1 \\ \text { Owner } & \text { “ } & \text { blk g Kangaroo, harness.1 } & 3 & 2 & 2 \\ \text { Owner } & \text { " } & \text { b g Centreville, harness.3 } & 2 & 3 & 3 \\ \text { C. M. Beckler } & \text { “ } & \text { br m Fanchon, harness..4 } & 4 & 4 & \mathrm{dr}\end{array}$

Time, $2.38 \frac{1}{4}, 2.36,2.37 \frac{3}{4}, 2.38 \frac{1}{2}$.

\section{JULY 30TH, 1869.}

\section{URBANA, III.}

JuLy 30.-Purse $\$ 1.000$ for horses that have never beaten 2.27. Mile heats, three in five, in harness.

$\begin{array}{lclllll}\text { M Colvin enters b s Pilot Temple.....2 } & \mathbf{1} & 2 & \mathbf{1} & \mathbf{1} \\ \text { M. Cavanagh } & \text { "6 } & \text { g g Silas Rich.......1 } & 2 & 1 & 2 & 2 \\ \text { T P. Roach } & \text { 4. } & \text { g g W. K. Thomas.... } & 3 & 3 & 3 & 3\end{array}$

Time, $2.26 \frac{1}{2}, 2.29,2.29 \frac{1}{2}, 2.33,2.35$.

JULY 30TH, 1869.

URBANA, III.

JuLY, 30th.-Parse \$175. Mile heats, best three in five, in harness.

J. H. Haight

W. Moore

F. N. Burrows

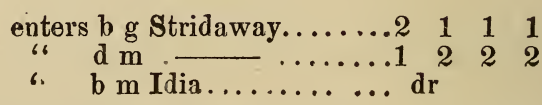

Time, $2.40 \frac{1}{2}, 2.36,2.36 \frac{1}{2}, 2.37$.

JULY 30TH, 1869.

\section{IAWRENCE, Kansas.}

JULy 30th.-Purse $\$ 75$, for horses that have never beaten 3.00 Mile heats, best three in five, in harness.

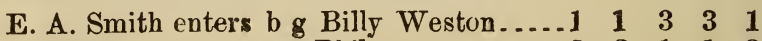

T. Clark " r g Phil...............

B. F. Aker « by Honest John......3 $\quad 2 \quad 2 \quad 2 \quad 3$

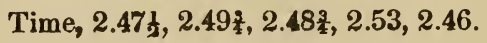


JULY 30TH, 1869.

\section{BUFFALO DRIVING PARK.}

JuLy 30th.-Purse \$200. Mile heats, best three in five, in harness.

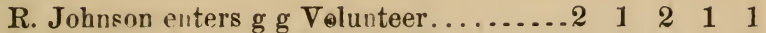

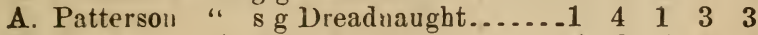

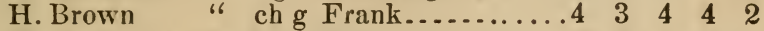

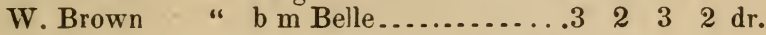

Time, $2.37,2.34,2.34 \frac{1}{2}, 2.35 \frac{1}{4}, 2.36 \frac{1}{2}$.

JULY 31sT, 1869.

DEXTER PARK, Chicago.

JuLy 31st._Sweepstakes $\$ 600$. Mile heats, best three in five, in harness.

J. Rockey enters b g Beaton................2 1 I 1

Owner " b m Belle of Cbicago.......

M. Doty g g Billy................

Time, 2.52, 2.48 $\frac{1}{2}, 2.49 \frac{3}{4}, 2.52 \frac{1}{4}$.

JULY 31st, 1869.

DUBUQUE DRIVING PARK, IOwa.

JULY 31st.-Purse and stake $\$ 300$. Mile heats, best three in five, in harness.

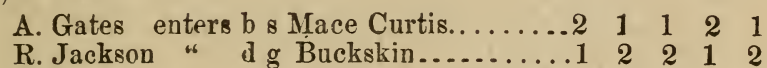

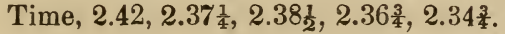

JULY 31st, 1869.

OIMAHA TROTTING PARK.

July 31st.-Purse $\$ 50$. Mile heats, best three in five, in harness.

W. Hodges enters b g Bay Jim............... 1

T. Palmer " s g Blind Billy..............2 2 \%

Time, 2.44, 2.43, 2.45 . 
JULY 31st, 1869.

RIDING PARK COURSE, Manchester, N. H.

JuLY 31st.-Match $\$ 800$. Mile heats, best three in five, in harness.

G. F. Nutter enters b g Martin Luther......... 1

W. A. K. Hoyt " $\mathrm{r} m$ Rosa.................2 22

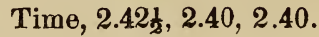

JULY $31 \mathrm{sT}, 1869$.

FASHION COURSE.

JuLY 31st - Sweepstakes $\$ 330$. - Mile beats, best three in five, in harness.

Mr. Haslett enters c $\mathrm{g}-\ldots \ldots \ldots \ldots \ldots \ldots \ldots$

Mr. Hill " $\mathrm{g} g-\ldots \ldots \ldots \ldots \ldots \ldots . .2 \quad 2 \quad 2$

Mr. McMara “ brg

Time, 2.52⿺ 1 , 3.52, 2.54.

AUGUST 2ND, 1869.

FASHION COURSE.

August 2nd.-Sweepstakes $\$ 300$. Mile heats, hest three in five, in barness.

Dr. Ogle enters b g Red Rover................ 1

H. Casey " blk g Black Dan................ 2

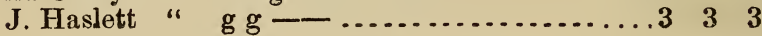

Time, 2.49. 2.44, 2.44 .

AUGUST 3RD, 1869.

RIVERSIDE PARK.

August 3rd.-Match $\$ 300$. Mile heats, bost three in five, in harness.

D. Bigley enters r g General Hooker..2 $2 \begin{array}{lllll}2 & 1 & 1 & 0 & 1\end{array}$

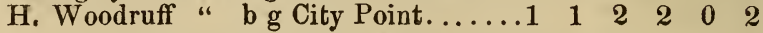

Time, 2.53, 2.53ł, 2.54, 2.54, 2.51ł. 
AUGUST 4TH, 1869.

PORTSMOUTH, N. H.

August 4TH.-Purse \$250. Mile heats, best three in five, in harness.

Owner euters Sallie...................... 1 1 1

Owner "Kite....................... 2 2

Owver " Lady Lawrence................. 3 3 3

Time, 2.47, 2.47, 2.47.

AUGUST 4TH, 1869.

SARATOGA.-Fair Grounds.

August 4th.-Purse $\$ 400$.-Mile heats, best three in five. in harness.

B. Mace enters $w$ g White Fawn............ 11

W. Borst * br g Old Put................2 $2 \quad 2$

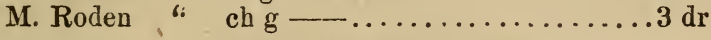

Time, $2.44,2,43 \frac{1}{2}, 2.43 \frac{1}{2}$.

AUGUST 5TH, 1869.

CINCINNATI, 0.

August 5th.-Purse \$ - Mile heats, best three in five, in harness.

Owner enters Lexington.................. 1

Owner " Live Oak.................2 2

Owner " Dolly.....................

Time, $2.47 \frac{1}{2}, 2.45 \frac{3}{4}, 2.47 \frac{1}{4}$.

AUGUST 6TH, 1869.

RIVERSIDE PARI.

AUG. 6th.-Handicap Race. Match $\$ 500$. Mile heats, best three in five

H. Bradley enters olk ge Black Harry (to wagon) 21111

W.H. Woodruff “ b g Centriville (in harness)..1 222

$\operatorname{Tim} \theta, 2.41 \frac{1}{4}, 2.40,2.38 \frac{1}{2}, 2.39$ 妾, 
AUGUST 6TH, 1869.

\section{SUFFOLK PARK COURSE.}

AUG. 6th.-Purse $\$ 500$-Mile heats, best three in five, in harness.

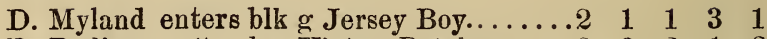

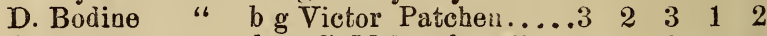

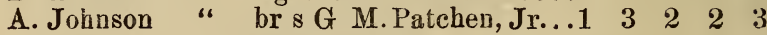

P. Daly

“ $8 \mathrm{~g}$ Harry D.........4 dis

Time, 2.39, 2.40, 2.39, 2.41, 241.

\section{AUGUST 7TH, 1869.}

EASTON, Pa.

AUG. 7th.-Match $\$ 100$. Mile heats, best three in five, in harness.

T. Newman enters s g Dr. Jackson.......... 1

H. Hartpence " b g Bill Jackson...........2 2

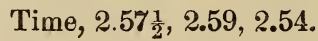

AUGUST 7TH, 1869.

COID SPRING TRACK, Milwaukee, Wis.

AUG. 7th.-Purse $\$ 500$. Free for all horses. Mile heats, best three in five, in harness.

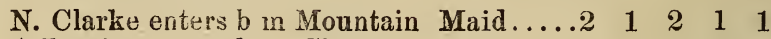

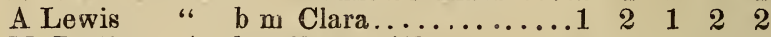

M. Pettit " b m Fanny Allen.........dr

Time, $2.30 \frac{3}{4}, 2.31 \frac{1}{4}, 2.33 \frac{1}{4}, 2.30 \frac{3}{4}, 2.32$.

AUGUST 10TH, 1869.

BUFFALO DRIVING PARK.

August 10th.-Purse $\$ 1,000$, for all horses that have never beaten 3.00. Mile heats, best three in five, in harness.
A. Lewis enters $g \mathrm{~g}$ Gray Hawk............. 1 1 1
W. Brown
" b m Ella Elwood............2 2
B. L. Rush
6. b m Mary P. Barnum........4 $4 \begin{array}{lll}3 & 3\end{array}$
L. McQuibbey “ d g Fuller Brown.......... 3 dis.
A. Patterson
b g Norwood...............dr.

Time, 2.38 $\frac{1}{2}, 2.42 \frac{1}{4}, 2.43 \frac{3}{4}$. 
AUGUST 10TH, 1869.

\section{BUFFALO DRIVING PARK.}

AUGUST 10th.-Purse $\$ 2,000$, for all horses that have never besten 2.34. Mile heats, best three in five, in harness.

T. Best enters br g Henry.............. 1

Wm. Brown "6 b g Dutchman................. 2

J. Jennings “ b s Anthony Waynu.......... 4 4 3

F. Howard " b s Tete Marhews...........7 34

S. R. Clırk " ch m Lizzie............... 55

D. Mace

R. Barnes

A. Patterson

J. Woodruff

b in Lydia Thompson........2 dis.

s g Frank...............6 dis.

g g Gray Prince..........

b g Listener..............

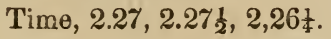

AUGUST I0TH, 1869.

MANCHESTER, NN. H.

Aug. 10th.-Purse $\$ 300$, for horses that have nerer beaten 3.00 . Mile heats, best three in fire, in harness.
J. Mooney
D. Bigley
H. Woodruff
W. O. Hopkins
enters b m Sally.............. $1 \quad 1$
F. Nichols
S. Hayes
D. C. Whittemore
J. Mooney
F. Jones
W. S. Briggs

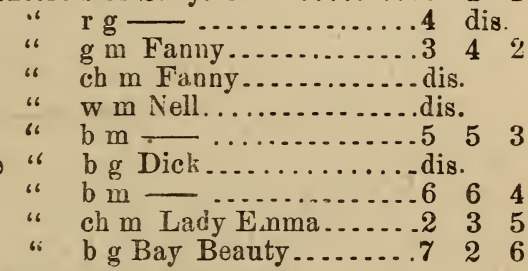

Time, 2.39, $2.41 \frac{1}{2}, 2.42$.

AUGUST 11TH, 1869.

MANCHESTER, N. H.

AUG. 11th.-Purse $\$ 300$, for horses that have never beaten 2.50 . Mile heats, best three in five, in harness.

H. Woodruff
F. Jones
S. Hayes
C. Mosher
D. Bigler
D. C. Whittemore
W. S. Briggs
L W. Ray

Time, 2.37, 2.4 $\frac{1}{2}, 2.39 \frac{1}{4}$. 
AUGUST 11TH, 1869.

MANCHESTER, N. F.

AUG. 11th.-Purse $\$ 500$ for horses that have never beaten 2.30. Mile heats, best three in five, in harness.

A. W. Worcester enters ch g McClellan....2 22111

H. Bradley

J. Copp

W. Locke

" ch g Black Walnut..41422

" b $\quad$ m Empress.......1 $3 \begin{array}{llllll} & 2 & 3 & 3\end{array}$

“g g m Nellie Locke...3 43344

Time, 2.33, $2.30 \frac{1}{4}, 2.35 \frac{1}{2}, 2.32 \frac{1}{2}, 2.32 \frac{1}{4}$.

AUGUST 11TH, 1869.

\section{BUEFALO DRIVIEG PARK.}

AUG. 11th. - Purse $\$ 10,000$, free for all horses. Mile heats, best three in five, in harness.
B. Doble
B. Daniels
enters b m Goldsmith Maid.......1 11
C. Champlain
D. Nace
" b m American Girl.........3 2 2
" br g Geo. Palmer..........2 33
" b m Lucy.............. dr

Time, 2.193 $2.19 \frac{1}{2}, 2.19$ 욘.

AUGUST 11тн, 1869.

\section{BUFFAIO DRIVING PARK.}

Aug. 11 th $\_$Purse $\$ 1,500$, for horses that have nerer beaten 2.40 Mile heats, best three in fivo, in harness.
A. Rille
enters br $\mathrm{g}$ Henry............ $1 \begin{array}{llll}1 & 1 & 2 & 1\end{array}$
W. Brown
" b s Young Blackbird... 3 2 21
O. A. Hiekock
Owner
H. W. Brown
W. Taggsrt
ch s St. Elmo.......... $7 \quad 6 \quad 5 \quad 3$
Frank Rolfe..... 5 3
“
“ ch g Frank..........2 7 \% 3 \%
O. W. Dinmick
“ b g Cayuga Chief. .....6 6 5 446

Time, 2.331 $\frac{1}{3}, 2.32,2.32,2.31 \frac{1}{3}$.

AUGUST 11TH, 1869.

\section{BUFFALO DRIVING PART.}

Aug. 11th.-Purse $\$ 3,000$, for horses that have never beaten 2.29. Mile hea ts, best three in five, in harness.

J. Dumas enters ch $m$ Idol.......... $4 \begin{array}{llllll}1 & 1 & 3 & 2 & 2 & 1\end{array}$

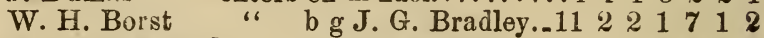

W. J. Lockwood “ br s Kirkwood...... 

J. Turner
enters b m Fanny Allen...1 6446644
C. H. Garretson " " g g Surprise.....2248834 dr
E L. Morris
P. IV ond
II. Rodẹn
b g N. B Palmer...3965 54 dr
" b g Wostern New
o. A. Hickock " b $\mathrm{m}$ Angeline..... $7578 \mathrm{dr}$
E. 'T. Howard " b g Gen'l. Scott...81099 dr
O. W Dimmick “ ch s Byron........ $\mathrm{dr}$

Time, $2.28 \frac{1}{4}, 2.27,2.29 \frac{1}{2}, 2.27 \frac{1}{4}, 2.24,2.26 \frac{3}{4}, 2.28 \frac{3}{4}$.

\section{AUGUST 12TH, 1869.}

\section{BUFFALO DRIVING PARK.}

AUiv. 12th.-Purse $\$ 1,000$, for horses that have never beuten $2-50$. Mile heats, best three in five, in harness.
T. Bist
enters b g Henry.............. I 1
O. W. Dimmick “ b m Lady Garfield.........4 22
O. A. Hickock " ch s St. Elmo............... 3 3 3
A. Lewis
Owner
" g $g$ Gray Hawk...........5 5 . 4
“ brm Brown Kitty..........2 4 4 5
A. Patterson " g g Gray Priace...........6 dis

Time, 2.324, 2.293, 2.343.

AUGUST 12TH, 1869.

MANCHESTER, N. H.

A UG. 12th.-Purse $\$ 360$, for horses that have never beaten 2.42 . Mile heats, best three in five, in haruess.
J. Mooney enters b $\mathrm{m}$ Sally................ 1
W. S. Briggs " b m Bay Beauty.............2 2
S. Hayes
L. W. Ray
" b g Martin Luther........... 3 3 3
" ch $\mathrm{g}$ Blu. her............. dis.
J. Alexander " blk g Black Joo.............. dis.

Time, $2.38,2.38 \frac{1}{4}, 2.40$.

AUGUST 13тн, 1869.

\section{BUFFALO DRIVING PARK.}

AUG. 13th.-Purse $\$ 5,000$, for horses that have never beaten 2.26. Mile heats, best three in five, in harness. 


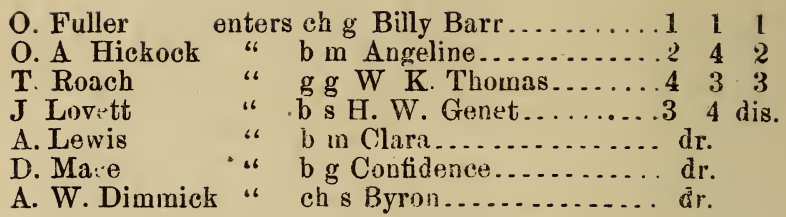

Time, $2.25,2.26_{4}^{3}, 2.2 \%$.

AUGUST 14TH, 1869.

GOLDEN GATE RIDING PARK, sain Francisco, Cal. Aug. 14th.-Purse $\$ 100$. Mile heats, best three in fire, to wagons.

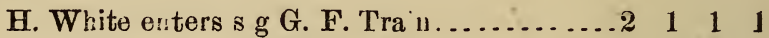

C. Lusk " s g Sorrell Charley...........

J. Eoff $\quad * \quad$ b g Stranger...............

Time, 2:48⿱3 $, 2: 49$, 2:49, 2:50.

AUGUST 15TH, 1869.

WATERTOWN DRIVING PARK.

Aug. 15th.--Match. Mile heats, best three in five, is haruess

White \& Doolittle enters b m Fanny Fern.....2 2111

Mr. Sternberg " b s Emperor........11222

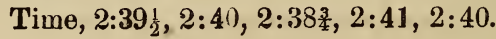

AUGUST 16Th, 1869.

FASHION COURSE, L. I.

Aug. 16th.-Match $\$ 500$. Mile heats, best three in five, in harness.

J. Cudney enters b m Minnie................... 1 1 1

J. Murphy “ b g George............... $2 \quad 2$

Time, 3:10, 2:58, 3.01.

AUGUS'T 17'TH, 1869.

OIL CIrY, Penn.

AUG. 17th.-Purse $\$ 500$. Mile heats, best three in five, in harness. 
Mr. Fowler enters s g Frank ............ 1121

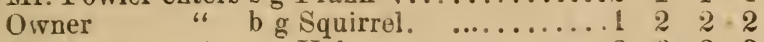

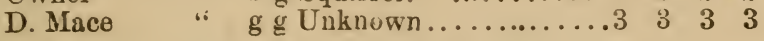

Time, 2:40, 2:38, 2:41, 2:43.

AUGUST 17 TH, 1869.

OLENTANGY PARK, Columbus, Ohio.

Aug. 17th.-Purse $\$ 150$. Mile heats, best throe in five, in harness.

Owner enters Gen. Grant.................. 1 1 1 3 1

Owner " Little Molly.................. 2

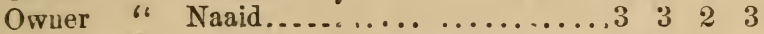

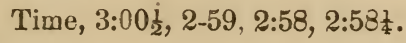

AUGUST 17TH, 1869.

ERIE DRIVING PARK, Pa.

AUG. 17th.-Purse \$65, fur Erie County hurses. Mile heats, best three in five, in harness.

Mr. Wadsworth enters g m Lady Wadsworth ... 1 1 11

M. Colby " b m Lady Custer........ 4 3 2

C. Dunbar “ $\quad$ rg Tormentor.........2 2

Brecht \& Jobnson “ b g Honest George........3 44

Time, 2.44, 2.48, 2.481 2 .

AUGUST 18Th, 1869.

ERIF DRIVING PARK, Pa.

ACG. 18th.-Purse $\$ 100$, for horses that bave never beateu 2.50 prior to August 18t, 1869. Mile heats, best three in five, in harness.

M. Colby enters b g Ploughboy............. $1 \quad 1 \quad 2 \quad 1$

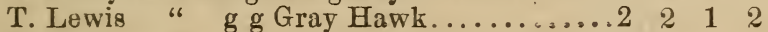

Owner

b $\mathrm{g}$

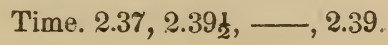

AUGUST 18TH, 1869.

OIL CITY, PA.

A UG. 18th.-Purse $\$ 2,500$. Free for all horses. Mile heats, best three five, in harness.

B. Daniels enters b m American Girl........ 1221

D. Mace “ b $m$ Lucy..............2 122

Time, 2.34, 2.28t, 2.32. 2.35. 
AUGUST 18TH, 1869.

RIVRRSIDE PARK, Mass.

AuG. 18th.-Gentlemen's Race. Purse $\$ 125$. Mile beats, best three in five, in harness.

Owner enters $\mathrm{b} m$ Cricket................. 1

Owner " b g Vermont Boy............... 2

Owner " $\quad \mathrm{s} g$ Columbus...................... 33

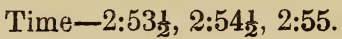

AUGUST 18TH, 1869.

OLENTAIJGY PARK, Columbus, Ohio.

A UG. 18th.-Purse $\$ 200$. Mile heats, best three in five, in harness.

J. Alexander enters $g \mathrm{~g}$ Johnny ...............

C. H. Olmstead " b g Frank..............

E. A. Lytle " s m Daisy................ 3

Time-2:45, 2:49, 2:49.

AUGUST 18TH, 1869.

COCHECO DRIVING PARK, Dover, N. H.

AUG. 18th.-Purse $\$ 200$, for all horses that have never beater 3:00. Mile heat, best three in five, in harness.

Owner enters Sally.................... 1

Owner " Lady Wilkes................... 22

Owier " Farmington Girl................ 3 3 3

Owner " "Tontine Belle................. dis

Time-2:47, 2:431 $, 2: 44$.

AUGUST 18Th, 1869.

COCMECO DEIVING PARK, Dover, N. H.

AUG. 18th. - Purse $\$ 150$, for horses that have never beaten 2:45. Mile heats, best three in five, in harness.

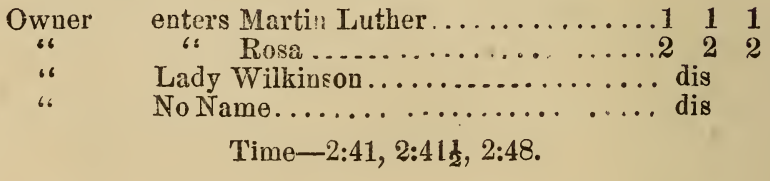


AUGUST 19TH, 1869.

NEW HAVEN, Conn.

Aug, 19th.-Purse $\$ 50$, for horses that have never beaten 3:00. Mile heats, best three in five, in haruess.
C. Dickeman
enters Sorrell Gelding........ 111
A. C. Whitson
Owner
"“ blk g Black Harry......2 2 2
“. b s Dick Parsons........3 dr

Time-2:58, 2:55, ₹:53.

\section{AUGUST 19TH, 1869. \\ NEW HAVEN, Conn.}

AUG. 19th.-Purse $\$ 100$, for horses that have never beaten 2:40. Mile heats, best three in five, in harness.
1. Taylor
C. Dickeman
C. M. Ferguson
A. C. Whitson

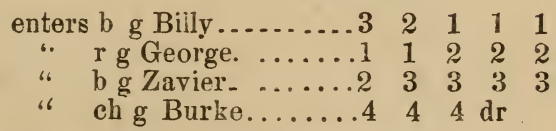

Time--2:45, 2:48, 2:46, 2:48, 2:423.

\section{AUGUST 19TH, 1869. \\ GLENBROOK, Cal.}

AUG, 19th. -Purse \$200. Mile beats, best three in fire, in harness.

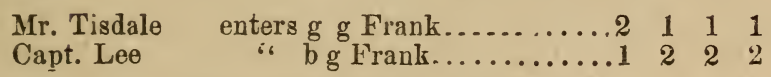

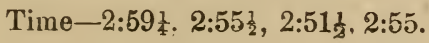

AUGUST 19rh. 1869.

NEW HAVEN, Conn.

AUG. 19th.-Purse $\$ 100$, for horses that have never beaten $2: 50$. Mile heats, best three in five, in harness.

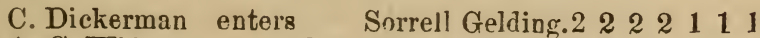

A. C. Whitson " blk g Canada Boy... $\begin{array}{llllllllllll} & 1 & 1 & 3 & 4 & 4\end{array}$

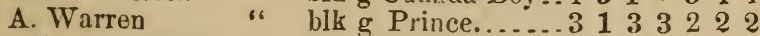

J. E. Flowers “ $\quad$ b g Tom Bradley.... 1444444433

Wm. Parker " b g Sir Tom......5 $5 \mathrm{dr}$

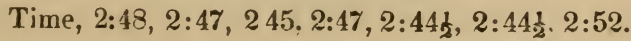


ACGUST 19TH, 1869 .

OLENTANGY PARK, Columbus, Ohio.

ACG. 19th.-Purse $\$ 100$. for horses thet have nerer bea pi 3:c0. Mile heats, beat three iv Iive, in harness.

C. H. Olmstead enters $\$$ \& Dick ........... 1 1 1

J. Alezauder " b m Lady..........2 2

Time-2:54, 3:02, 3.00.

ACGLST 19TH, 1969.

BRATTLEBORO, Vt.

- AcG. 19th.-Purse \$200. Mile beats, best three in fire, in harnes:.

W. O. Hopkins

W. E. Elliot

G. Ware

L. Putnams

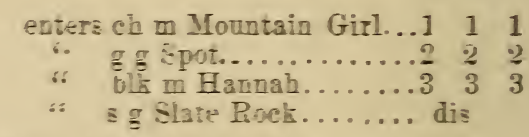

Time-2:25, 2:40, 2:42.

AUGUST 19TH, 1869.

ACG. 19th.-Purse $\$ 250$, for horses that hare never beateu 2:50. Mile heats, best three in fire, in harness.

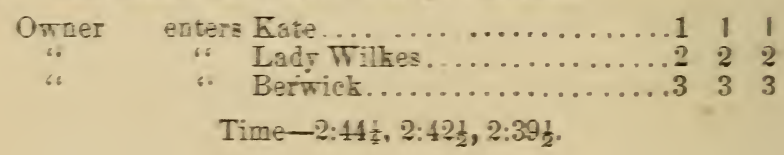

AUGUST $19 \mathrm{TH}, 1869$.

COCHECO DRIVING PARB, DOVE, N, H.

AtG. 19th.-Sweepstakes. Mile heats, best three in fire, in haruess.

Owner enters Sally ................... 1

Omner ". Porlad Girl ....................... 2

Owner "Sterida..........................

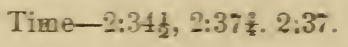


AUGUST 19Th, 1869.

ST. CATHARINE'S, Canada West.

AvG. 19th.-Purse $\$ 150$, for Canada horses that bave never beaten 3:00. Mile heats, best three in fire. in harness.

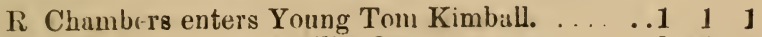

Owher

Owner

Owlier

“ Urillia Queen...........2 4 \%

“ Emily Fuster.............. 3 "̈ 3

“ Bay Gelding............4 2 dis.

Time-2:43, 2:47, 2:45.

AUGUST 20TH, 1869.

MYSTIC RIDING PARK, Mass.

AvG. 20th.-Purse and stake $\$ 2,500$. Mile heats, best three in five, in haruess.

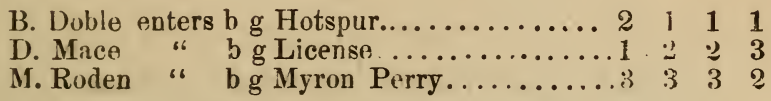

Time-2:27, 2:24, 2:25, 2:25 $\frac{1}{2}$.

AUGUST 2〔TH, 1869.

UTICA DRIVING PARK.

Aug. 20 h.-Purse $\$ .00$. Mile heats, best tbree in five, in harness.

Mr. Dirbey enters b g Rolla Golddust.......

J. Mh! phy “ b g Bradley..............

Time-2:35, 2:4(i, : : 42, 2:40.

AUGUST 21st, 1869.

SUFFOLK PARK, Philadelphia, Pa.

Aug. 21st.-Purse 500 . Mile beats, best three in five, in harness.

$\begin{array}{lllll}\text { W. H. Doble enters b g Hop } \ldots \ldots \ldots \ldots \ldots \ldots \ldots 1 & 1 & 1 \\ \text { J: Levitt } & \text { g }\end{array}$

Time-2.34, $242,2.47$. 
AUGUST 21sT, 1869.

COCHECO DRIVING PARK, Dover, N. H.

Aug. 21st-Purse $\$ 125$, for New Hampshire horses that bave never beaten 3.00. Mile heats, best three in five, in harness.

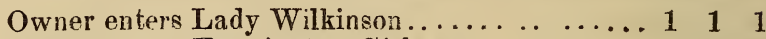

Owner " Farmington Girl.............2 22

Time-2:49, 2:53 2:50.

AUGUST 23RD, 1869.

SARATOGA, N. Y.

Adg. 23rd-Purse $\$ 3,000$. Mile beats, best three in five, in harness.

S. MeLaughlin enters $b \mathrm{~g}$ Mountain Boy,........ 1

D. Pfifer " b m Lady Thorn.........2 2

. Time-2:27, $2: 24 \frac{1}{4}, 2: 25 \frac{1}{2}$.

AUGUST $24 \mathrm{TH}, 1869$.

FAMPDEN PARK, Springfield, Mass.

AvG. 24th.-Purse $\$ 700$, for horses that have never beaten 2.38 . Mile heats, best three in five, in harness.
D Bigley
enters b m Fanny.
533111
M Riden
E C. Robinson
S E Stowel!
H. A. White

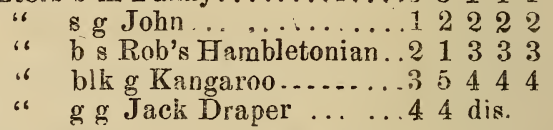

Time, 2:38 $\frac{3}{4}, 2: 41,2: 41,2: 42 \frac{3}{4}, 2: 39$.

AUGUST 24TH, 1869.

FAMPDEN PARK, Springfield, Mass.

AUG. 24th.-Purse $\$ 00$, for horses that have never beaten 300 . Mile heats, best three in five in harness.
M. Carroll
enters $b$ g Prince ............ 1111
A. C. Buckwatter " blk g Antharcite........6 82
J. H Phillips " ch in Fanny Fern....... .2 33
M. Bain
E. Savage
S E. Stowell
R. Reamer
M. Ruden
A. Roberts
" ch g Palmyra Maid.....4 24
“6 b m Dexter" Girl.........7 75
" br m-
" ch g _......... 4 4 7
" b g Paul Pry...........5 6 \&
" blk $m$ Neliy............ dis

Time, 2:42, 2:43, 2:41. 
AUGUST 25TH, 1869.

\section{HAMPDEN PARK, Mass.}

Aug 25 th.-Purse $\$ 500$, for horses that never trotted better thiln 2:50. Mile heats, best three in five, in harness.
H. A. White
S. Hayes
T. R Loomis
R. Reamer
C. Mosher
E. Savage
G. P. Carpenter
M. Roden
euters $\mathrm{g} g$ Jack Draper......... $1 \quad 1 \quad 1$
'b g Prince ............2 $22^{\prime} 2$
" br m Lady Wilkes........ 3 3 3
* b g Eolus (formerly Unclo Eben)..............dis
" c $g$
". blk m Black Betty....... dis
6. b m Dexter Girl....... . . dis
‘. blk g Capt. Dewey ...... dis
" Paul Pry...........dis
Time-2:4I, 2:40, 2:46.

AUGUST 25'TH, 1869.

JAMESTOWN, IN. $X$.

AUG.25th.-Purse $\$ 200$, for horses that have never beaten 2:38. Mile heats, best three in five, in harness.

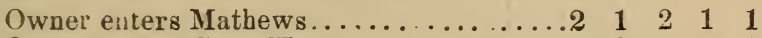

Owner "Gray Hawk.............

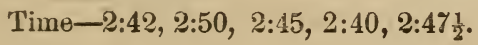

\section{AUGUST 25TH, 1869.}

\section{MIDDLETOWN, N. Y.}

AUG. 25th.-Purse \$—, for hor'ses that have never beateit 2:47, Mile heats, best three in five, in harness.

Jesse R Woods enters b m Mills Mare ..... . 11

W. C. Trimble

J. A. Decker

M. L. Wellers

W. Bodine

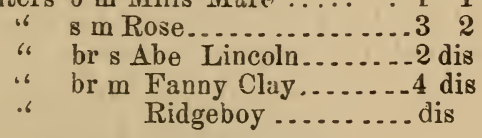

2

Time-2:47, 2:39, 2:42.

AUGUST 25\% 1869.

MIDDLETOW $N, N, Y$.

A UG. 25th. $\longrightarrow$ Purse \$-, for tuur year old stallions. Mile heats, in harness. 
John Mapes enters Bay Hambletonian. .......... 1

Wm. Homan " Bay Stallion................2 2

J. M. Mills “ Chorose..................2 2

Time-3:04, 2:51.

AUGUST 26TH, 1869.

HAMPDEN PARK, M:ss.

AUG 26th.-Purse $\$ 1,000$, for all horses that have never beaten 2:30. Mile heats, best three in five, in harness.

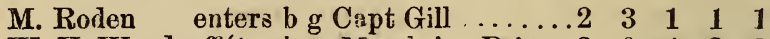

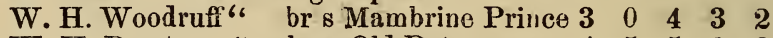

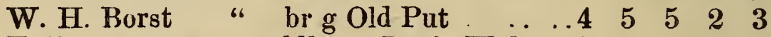

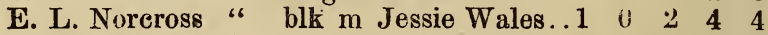

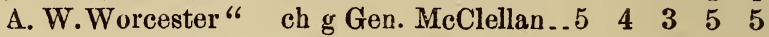

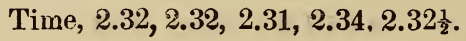

AUGUST 26тн, 1869.

MIDDLETOWN, N. $Y$.

AUG. 26th.-Purse $\$ 150$, for horses owned in Orange County that have never beat 3:00 previous to August 1st, 1869. Mile heats, best three in five, in harness.

W. C. Trimble
G. Lobdell
J. R. Wood
H. C. Bishop

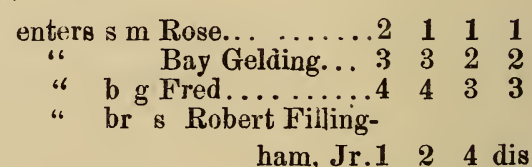

ham, Jr.1 $2 \quad 4$ dis

Time, 2:51, 2:47, 2:51, 2:51.

AUGUST 26тн, 1869.

MIDDLETOWN, N. Y.

AvG. 26th.-Purse $\$ 150$, for stallions owned and kept for service in Orange County. Mile heats, best three in five, in harness.

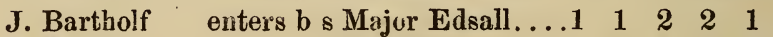

$\begin{array}{llllllll}\text { J. McGee } & \text { "b } & \text { b Honesty.......... } & 2 & 2 & 1 & 3 & 2\end{array}$

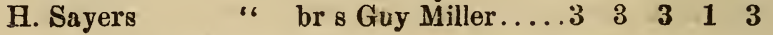

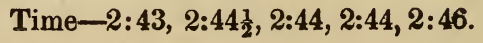




\section{AUGUST 26TH, 1869. MIDDLETOWN, N, $\mathbf{Y}$.}

Ava. 26th.-Purse $\$ 300$, for horses owned in Orange County, that have never beaten 2:35. Mile heats, best three in five, in harness.

J. McKee

W. D Smith

W. Doremus

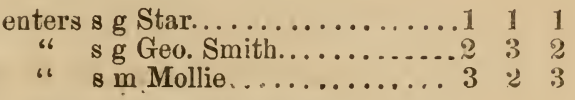

Time-2:39, 2:40, 2:41.

\section{AUGUST 26rн, 1869.}

CLINTON, Iowa.

AUG. 26th.-Purse $\$ 75$, for horses that have never beaten 3,00 Mile heats, best three in five, in harness.
W. W. Hamilton
enters b g Billy Hamilton.2 2111
C. W. Phillips
M. J. Lewis
J. Martin
A. F. Way
" 8 g Rubber.......112222
" s g Lacey ........ dis
“ Frank Dunlap.. dis
“ s g Rutland Boy... dis

Time-2:43, 2:39, 2:421, 2:44, 2:421.

\section{AUGUST 27тH, 1869.}

\section{CLINTON, Iowa.}

AuG. 27th.-Purse $\$ 100$, for horses that have never beaten $2: 45$. Mile heats, best three in five, in hariess.

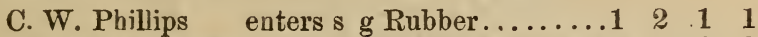

$\mathrm{W}$. W. Hamilton "“ b g Billy Hamilton..3 10.3

J. Rook

Joo Bennett....2 322

Time-2:38 $\frac{1}{2}, 2: 43 \frac{1}{2}, 2: 43 \frac{3}{4}, 2: 44$

\section{HAMPDEN PARK, Mass.}

AUGUST 27TH, 1869.

AUG. 27th.-Parse $\$ 400$. Mile heats, best three in five, in harness.

D. C. Pitkin enters b g Tom Thumb........
C. Mosher

Time-2:57 $2: 55,2: 43$. 


\section{HAMPDEIN PARK, Mass. \\ AUGUST 2\%TH, 1869}

AvG. 27th.-Purse $\$ 1,000$. Mile heats, in harness.

M. Roden enters b g Capt. Gill...........2 1 1

J.J. Bowen " ch g Gen'l. McClellan.......1 2 "2

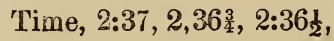

AUGUST 28TH, 1869.

COPAKE DRIVING PARK, N. $Y$.

AUG. 28th.-Purse $\$ 100$. Mile heats, best three in five, in nararness.

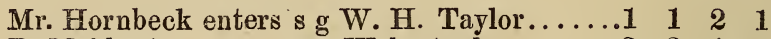

R. Mabbott ". $8 \mathrm{~g}$ Webertuck.........

J. Paulding " $\quad$ s s Burger............ 2 2 3 3

Time-2:31 $\frac{1}{2}, 2: 32 \frac{1}{2}, 2: 33 \frac{1}{2}, 2: 32 \frac{1}{2}$.

AUGUST 28TH, 1869.

CAMBRIDGE DRIVING PARK, N. Y.

AUG. 28th.-Purse $\$ 100$. Mile heats, best three in five, in harness.

$\begin{array}{lcccc}\text { Mr. Dougrey } & \text { enters George Taft } \ldots \ldots \ldots \ldots .1 & \mathbf{1} & \mathbf{1} \\ \text { Hickey Brothers } & \text { " } & \text { Billy Craver } \ldots \ldots \ldots \ldots .2 & 2 & \mathbf{2} \\ \text { Mr. Keeler } & \text { " Dan................ } & \mathbf{3} & 3\end{array}$

Time-2:50, 2:48, 2:45.

AUGUST 28rH, 1869.

PROSPECI PARK COURSE, I. I.

Aug. 28th.-Purse $\$ 4,000$. Mile heats, best three in five, in harness.

J. McMann enters b m Lady Thorn ........... 1 1 1

B. Doble " b m Goldsmith Maid......... 2 2 2

B. Daniels " " b m American Girl..........2 $3 \quad 3$

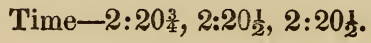

AUGUST 30TH, 1869.

CLINTON, IOWa.

AUG 30th.-Purse $\$ 300$, free for all horses. Mile heats, best three in five in harness. 


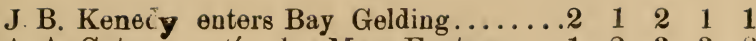

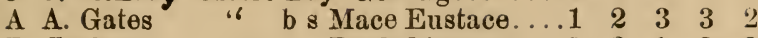

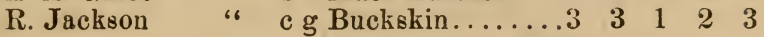

Time-2.34 $\frac{3}{4}, 2.35,2.33,2.38,2.31 \frac{1}{2}$.

AUGUST 31sT, 1869.

SUFFOLK PARK COURSE, Penn.

Avg. 31st.-Match. Milo heats, three in five in hazness.

W. H. Doble enters b g Hop . . . . . . . . 1

D. Bodine " b g Victor Patchen......2 $0 \begin{array}{llll}0 & 2 & 2\end{array}$

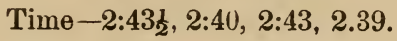

AUGUST 31st, 1869.

\section{POINT BREEZE PARK.}

AUG. 31st.-Purse $\$ 500$. Mile heats, best three in five, in harness.

A. Nugent enters b g George................ 1

P. Daley " 8 g Harry D............... 2

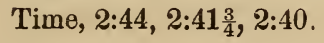

AUGUST 31sT, 1869.

\section{RIVERSIDE PARK, Mass.}

AUG. 31st.-Purse $\$ 250$, for horses that never have beaten 2:42. Mile heats, best three in five, in harness.

D. Bigley enters b m Kate............... 1

D. W. Begler "6 g g Sorrell Jobn............. 4 4

S. Hayes “ br m Lady Wilkes.. . ......2 2

H. Bradley “ b m Jennie Spencer.......... 33 . 4

S. Emurson " br g Fränk............ ..4 dis.

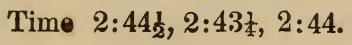

SEPT. 1ST, 1869.

LANCASTER PARK, Penn.

SEPT. 1st--Purse $\$ 2,000$. Mile heats, best three in five, in harness.

B. Doble enters b in Goldsmith Maid....... . . 11 1 1

B. Daniels " American Girl...........2 22

Time, $2: 31 \frac{1}{2}, 2: 26 \frac{1}{2}, 2: 30 \frac{3}{4}$. 
SEPT. 2ND, 1869.

MT. VERNON, OHIO.

SEPT. 2nd.-Match, $\$ 500$. Mile heats, best three in five, in harness.

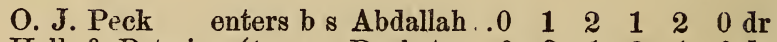
Hall \& Potwin "

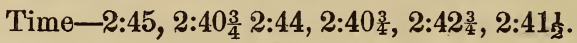

SEPT. 3RD, 1869.

CANASTOTA DRIVING PARK, N. Y.

SEPT. 3rd.-Purse \$200. Mile heats, best three in five, in harness.
J. Feek
J. Schuler
C. J. Elenwood
enters $b$ m Kitty ............. 1
"6 b m Lady Burke........2 2 .2 2
" b m Oneida Maid......... 3 3 3

Time, $2.41 \frac{1}{2}, 2.45 \frac{3}{4}, 2.41 \frac{1}{4}$.

SEPT. 3RD, 1869.

PROSPECT PARK COURSE, I. I.

SEPT 3rd.-Purse $\$ 2000$. Mile heats, best three in five, in harness.

J. McMann enters b m Lady Thorn.......... 1 1 1

S. MeLaughlin " b g Mountain Boy ........2 22

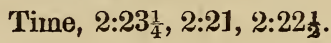

SEPT. 3RD, 1869.

MYSTIC PARK, Mass.

SEPT. 3rd.-Purse and stake $\$ 500$. Mile heats, best three in five, in harness.

W. H. Borst enters blk $g$ Old Put.... 3 J $3 \begin{array}{llll}3 & 2 & 1 & 1\end{array}$

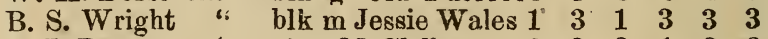

$\begin{array}{lllllll}\text { J. J. Bowen " oh } \mathrm{g} \text { McClellan....2 } & 2 & 2 & 1 & 2 & 2\end{array}$

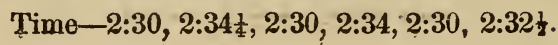


SEPT. 3RD, 1869.

\section{POINT BREEZE PARK.}

SEPT. 3rd.-Purse\$—. Mile hents, best three in fire, in harness.

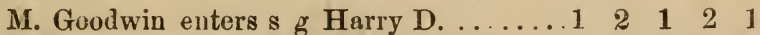

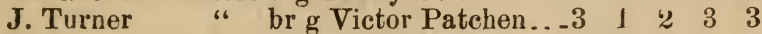

A. Johnson " bk s Geo. M. Patchen.2 $30301 \%$

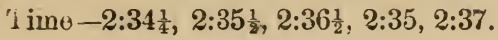

SEPT. 3RD, 1869 .

HURSTSVILLE, N. Y.

SEPr. 3rd.-Purse \$-—, Mile heats, best three in tive, in harness.

Owner
M. Remer

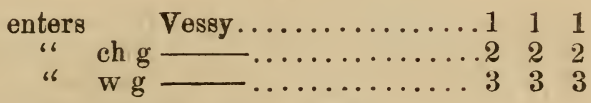

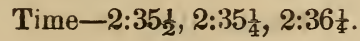

SEPT. 4TH, 1869.

NEWBURGH, N. Y.

SEPT. 4th.-Purse $\$ 50$, for four year old stallions. Mile heats. in harness.

J. S. Mapes enters b s Mapes Hambletonian... ...1 1

W. A. Homan " b s

J. N. Mills “ b s Chorose................

Time, 2.52, $2.56 \frac{1}{4}$,

SEPT. 4TH, 1869.

INEWBURGH, IN. Y.

SyPT. 4th.-Purse $\$ 70$, for five year old stallions. Mile bests, in harness.

J. Hoyt enters br s Abe Lincoln............ 1

W. H. Shelly "“ b s Henry Clay.......... 22

J. D. Smith “ b s _ ............dr

Time, 2:48, 2:47. 


\section{SEPT. 4TH, 1869. \\ NEWBURGH, $\mathbf{N}, \mathbf{Y}$.}

SEPT. 4th.-Purse $\$ 100$, for mares and geldings, six years old and upwards. Mile heats, in harness.

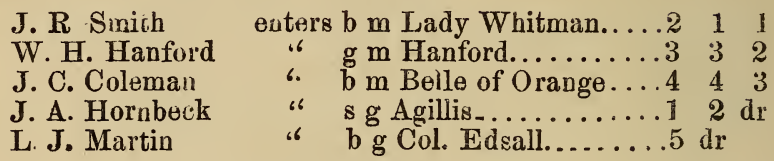

Time-2:39, 2:41, 2:41.

SEPT. 4TH, 1869.

NEWBURGH, N. Y.

SEPT. 4th.-Purse $\$ 70$, for mares and geldings, five years old. Mile beats, in hirness.

Alden Goldsmith

J. C. Howland

J. S. Edsall

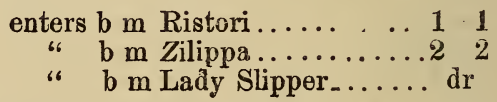

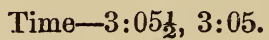

SEPT. 4TH, 1869.

NEWBURGH, N. $\boldsymbol{Y}$.

SEPT. 4th.-Purse $\$ 160$, for stallions six years old and upwards, owned or kept for service in Orange County. Mile heats, best t ree in five, in harness.

J. H. Bertholf

D. Sayre

H. C Bishop

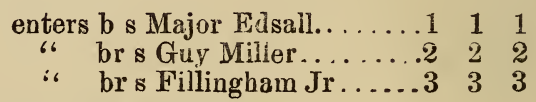

Time-2:43, 2:42, 2:421

SEPT. 4TH, 1869.

NBWBURGH, N. Y.

SEPT. 4th,-Purse $\$ 200$, for horses owned in Orange County. Mile beats, best three in מ̊ve, in harness.

J. R. Smith

L. H. Bodine

W, B. Smith enters b m Laãy Whitman..... 1 1 1

" s m Mollie. ..........2 2 dis

" s g Geo._Smith........3 dr

Time, 2:43, 2:43, 2:32. 
SEPT. 4TH, 1869.

NEWBURGH, N. Y.

SEPT. 4th.-Purse $\$ 100$, for horses owned in Orange County, that have never beaten 2:4.j. Mile heats, best three in five, in harness.

J. R. Wood

W. B. Smith

G. N Bell

L. Eager

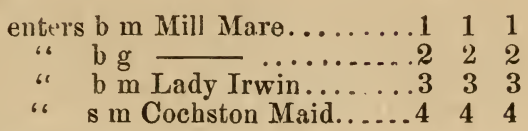

Time, 2:46, 2:45, 2:50.

SEPT. 4TH, 1869.

\section{CANASTOTA DRIVING PARK.}

SEPT. 4th-Purse $\$ 100$, for horses that have never beaten 242 . Mile heats, best three in five, in harness.
A. Crow
C. Fuley
B G. Foster.
erters g g Messenger Boy,....... 111
" b $\mathrm{m}$ Belle of Oneida.......2 32
"6 b m Temperance Girl.....3 23

Time, $2: 4 \gamma_{\frac{1}{2}}, 2: 43 \frac{1}{2}, 2: 44$.

SEPT. 7TH, 1869.

\section{SYRACUSE DRIVING PARK.}

SEPT. 7th.-Purse $\$ 300$, for horses that bave never beaten 3.00. Mile neats, best three in five, in harness.
A. Crow
enters $\mathrm{g} g$ Messenger Boy....5 551121
H B. Haley
" br m -.........3 133312
G. S. Hosmer
$46523 \mathrm{dr}$
C. B. Gould
E. H. Linsey
" br m
$23444 \mathrm{dr}$
F. Haight
br \& Shadow
$64255 \mathrm{dr}$
" b b g
" b $\mathrm{m}$ Fearless......... dis

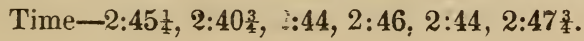

SEPT. 7TH, 1869.

\section{SYRACDSE DRIVIING PARK.}

SePr. 7th.-Purse $\$ 500$, for horses that have never beaten $2: 34$. Mile heats, best tbree in fire, in harness.

J. H Burke enters $\mathrm{s} g$ Ned Jones ........... 1

B. W. Kelly "“ blk s Kelly Horse.........2 22

J. Shuler " b m Lady Burke........... $3 \quad 3 \quad 3$

Time, $2: 37 \frac{1}{4}, 2: 45 \frac{1}{2}, 2: 41 \frac{3}{4}$. 
SEPT. 7TH, 1869.

NARRAGANSETT PARK.

SEPT. 7th.-Hiram Woodruff stakes, for colts and fillies. Mile heats, in harness. Winner Spirit of the Times stakes to go to wagon.

B. S. Wright enters ch g Fearnaught, Jr., by Fearnaught........................

Geo. C. Hitcheock enters b f Highland Ash, by

Ashland (to wagon) $\ldots \ldots \ldots \ldots \ldots \ldots \ldots \ldots 12$

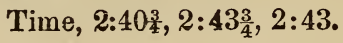

\section{SEPT. 7TH, 1869. \\ POINT BREEZE PARK.}

SEPT, 7th.-Purse $\$ 000$, for horses that never beat 3:00. Mile heats, best three in five, in harness.

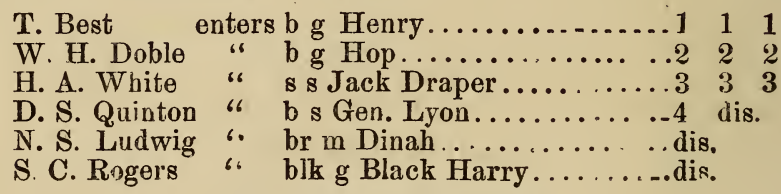

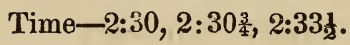

SEP Г. 7TH, 1869.

POIN'T BREEZE PARK.

SEPT. 7th.-Purse $\$ 2,000$, for horses that have never beaten 2:25. Mile heats, best three in five, in harness.

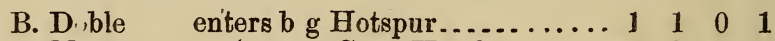

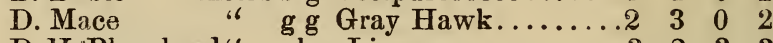

D. H.'Blanchard" ch g License...........

Time-2.28 $\frac{1}{2}, 2.25 \frac{1}{2}, 2.28 \frac{3}{4}, 2.29 \frac{1}{2}$.

SEPT. 8TH, 1869.

UNION PARK, Sacramento, Cal.

SEPT. 8th. - Purse $\$ 400$. Mile heats, best three in five.

B. Rice enters b m May Queen (to wagon). 12121 S. E. Whitehead "6 b g Regulator (in harcess)... 3121212 J. L. Eoff " $\mathrm{g}$ \& Paddy McGee (in har.).2 33 dis.

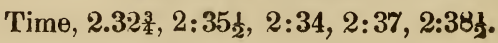


SEPT: 8TH, 1869.

UNION PARK, sacramento, Cal.

SEPT. Sth.-Purse $\$ 250$. Mile heats, best three in five, in harness, for horses that have never beaten 2:40.

H. L. Spenser enters b s Alexander.........

D. Demnison " b s Friday McCracken..2 1212

H. W. Searle " ch m Bracelet.........dis.

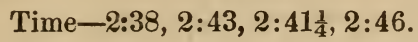

SEPT. 8TH, 1869.

UNION PARK, Sacramento, Cal.

SEPT. 8th.-Purse $\$ 250$, for three year olds. Mile heats, in harness.

J. Sessions enters g m California Maid, by Hamilton Chief................................. 1 C. H. Sisson enters s m Stockton Maid, by Chieftain.2 2

H. W. Searle “" s g Onward, by Chieftain........3 3

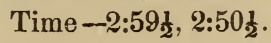

\section{SEPT. 8TH, 1869. POINT BREEZE PARK.}

SEPT. 8th.-Purse $\$ 1,000$, for horses that have never beaten 2:45. Mile heats, best three in five, in harness.

T. Best enters b g Henry................ 1

H. A. White " s s Jack Draper...............

W. H. Doble “ br m Lizzie Keller..........2 3

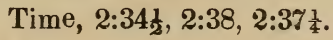

SEPT. 8TH, 1869.

\section{POINT BREFIZE PARK.}

SEPT. 8th.-Purse $\$ 2,000$, for all horses that have never bealen 2:30. Mile lieats, best three in five, in harness.
D. Mace
W. H. Borst
E L. Norcross

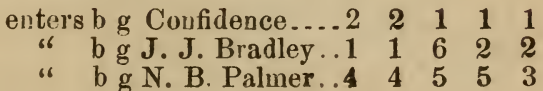




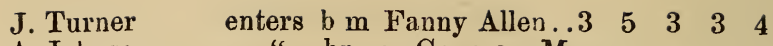

A. Johnson

$$
\text { Patchen, Jr....6 } 6 \begin{array}{llll}
6 & 4 & 6 & 5
\end{array}
$$

W. H. Woodruff “ br g Mambrino

$$
\begin{array}{cccccc}
\text { Prince.......... } & \ldots & 3 & 4 & 6
\end{array}
$$

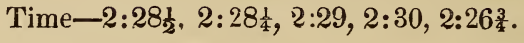

\section{SEPT. 8TH, 1869. \\ FRANKLIN COUNTY, Ohio-- Eair Grounds.}

SEPT. 8th.-Purse $\$ 60$, for horses that have never beaten 3:00. Mile heats, best three in five, in harness.

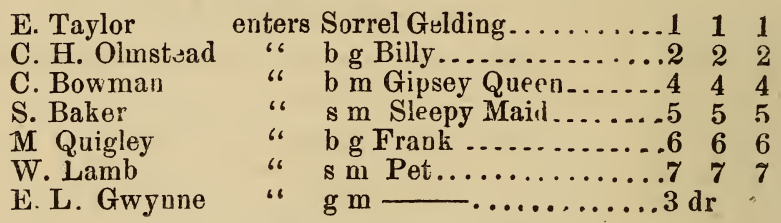

Time, 3:03, 2:56, 2:55.

SEPT. 8TH, 1869.

FRANKLIN COUNTY, Ohio.-Fair Grounds.

SrPT 8th.-Purse $\$ 100$. Mile heats, best three in five, in harness.

Owner enters b m Fanny................. 11

J. W. Shapley " g g Johnnie................. 2 2

Time, 2:47, 2.403 $2: 43 \frac{1}{4}$.

SEPT. 8TH, 1869.

ROCK ISLAND, $\mathrm{Il}$.

SEPT. 8th.-Purse $\$-$, for horses that have never beaten 2:5 Mile heat, best three in five, in harness.

B. Hershey enters b s Royal George........... 1 ।

T. P. Roach "
Owner

Time-2:553, 2:55, 2:52. 
SEPT. 8TH, 1869.

ROCK ISLAND, IIl.

SEPT. 8th.-Purse $\$ 175$, for horses that have never beaten $2: 35$. Mile heats, best three in five, in harness.

S. H. Perry enters b g Alexander

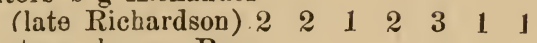

T. P. Roach enters b m Rosa

$\begin{array}{llllllll}\text { Bonheur........... } & 1 & 2 & 3 & 3 & 2 & 2\end{array}$

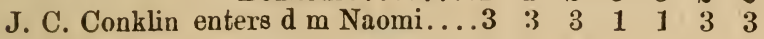

Time-2:39, 2:383 $, 2: 36,2: 35,2: 38,2: 42,2: 37$.

SEPT. 8TH, 1869.

SYRACUSE DRIVING PARK.

SEPT. 8th.-Purse $\$ 350$, for horses that have never beaten 2:50. Mile heats, best three in five, in harness.
A. J. Feek
B. W. Kelly
J. B. Berlen
F. Haights
E. H. Lindsley
A. Cloughs
enters b m Lola Montes...... 4 1 11
“ blk s Kelly Horse..... 1 3 32

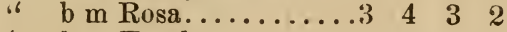
6. b m Fearless........2 $24 \quad 4 \quad 4$
"6 b $g-\ldots \ldots \ldots 5$ dis
. $\mathrm{g} g$ Messenger Boy.... dis

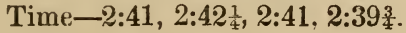

SEPT. 9TH, 1869.

ROCK ISLAND, III.

SEPT. 9th.-Purse $\$ 100$, for horses that have never beiten $\mathbf{2 . 4 5}$. Mile heats, best three in five in harness.

W T. Dixon

'I'. P. Roach

B. Hershey

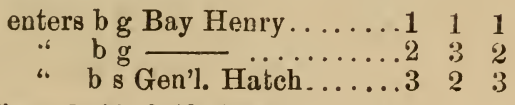

Time, 2:46, 2:42, 2:43.

SEPT: 9TH, 1869.

\section{ROCK ISLAND, III.}

SLPT. 9th.-Purse $\$ 500$. Mile heats, best three in five, in harness.

M. Colvin

J. Rockey

C. S. Bliss

A. A. Gates

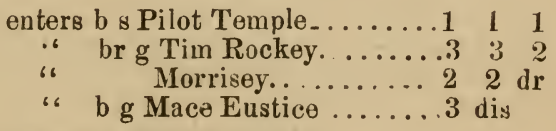

Tine, 2:29, 2:30 2 , 2:31. 
SEPT. 9TH, 1869.

FORREST CITY PARK, Portland, Me.

SEPT. 9th.-Purse $\$ 300$, for colts four years old. Mile heits, in barness

s Farmer

J. May

G. M. Bailey

enters b g John Franklin.......... 1

" blk g Uncle Abe.. ........2 2 2

" blk $\mathrm{m}$ Violetta............... 3

Time-2:52, 2:49.

SEPT. 9TH, 1869.

FORRIST CITY PARK, Portland, Me.

SEPT. 9ti.-Purse $\$ 250$. Mile heats, in harness.

Gilbreth enters Knox................ 1

Owner " ch g Gen'l. McClellan..........2 2

Time-2:34, 2:36.

SEPT. 9TH, 1869.

FORREST CITY PARK, Portland, Me.

SEPT. 9th.-Purse $\$ 15$ \%, for horses that have never beaten 2.38 Mile beats, best three in five, in harness.

R. L. Flouler

J. H. Lyford

C. B. Trask

H. A. Hall

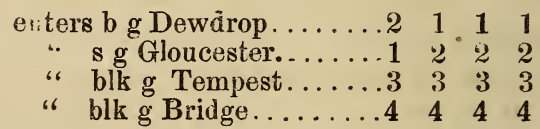

Time, 2:41, 2:41, 2:42, 2:41

SEPT. 9TH, 1869.

FORREST CITY PARK, Portland, Me.

SkPT. 9th. $\$ \$ 500$, for horses that have never beaten 1.45. Mile heats, best three in five, in hrrness.
O. O. Crosby
G. V. Gordon
H. A. Hall
J. Shaw
enters g g Plato. ......... 1
" b g Lonesome Reuben.2 \& 2
" b m Rosa.......... dr
" g g Beauregard........ dr

Time, 2.46, 2.46, 2.50 . 
SEPT. 9тн, 1869.

\section{POINT BREEZE PARK.}

SEPT. 9th.-Purse $\$ 1,000$, for horses that have never beaten 2.35. Mile heats, best three in five, in harness.

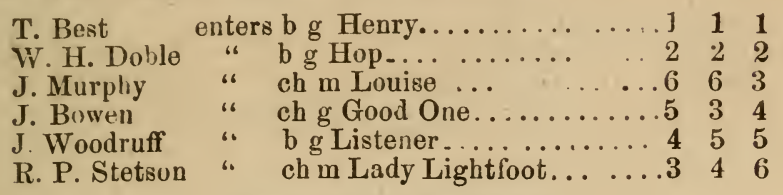

Time, $235 \frac{1}{4}, 3.34 \frac{1}{4}, 2.33 \frac{1}{2}$.

SEPT. 9TH, 1869.

POINT BREEZE PARK.

SEPT. 9th.-Purse $\$ 2,500$, for all horses. Mile heats, best thice in five, in harness.

J. McMann enters b m Lady Thorn .........

B. Daniels “ b m American Girl.....

B. Doble “ b m Goldswith Maid.......2 223

Time, 2.213 $2.19 \frac{3}{4}, 223 \frac{1}{4}$.

SEPT. 10TH. 1869.

UNION PARK COURSE, sacramento, Cal.

SEPT. 16th.-Purse \$-. Mile heats, best three in five, iu haruess. -

C. W. Moulthrop enters g m Marysville Queen - $1 \quad 1 \quad 2 \quad 1$

H L. Spencer “ b s Alexander........2 22 ( 12

Time, 2.423, 2.40, $2.45 \frac{3}{4}, 2.45$.

SEPT. 10TH, 1869.

ST LAWRENCE CO., N. $Y$.

SePT. 10th. -Purse $\$ 125$, for horses that hav never beaten 2:50. Mile heats, best three in five, in harness.

J.H. Phillips enters b g Billy (for Ace of

Diamonds $) \ldots \ldots \ldots \ldots \ldots \ldots \ldots \ldots \ldots \ldots \ldots$

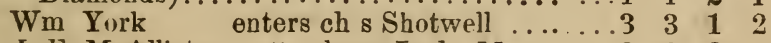

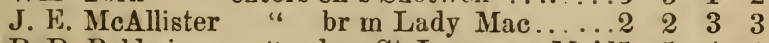

R. D Baldwin “ b m St. Lawrence Maid5 5040

$\begin{array}{llllll}\text { E. Howard " br s Ira Allen........ } 4 \text { 4 } & 5 & 5\end{array}$

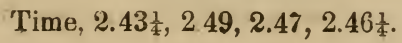


SEPT. 10TH, 1869.

\section{MENDOTA, Ill.}

SfPT. 10th.-Purse $\$ 500$. Mile heats, best three in five, in harness.

Owner enters Ida May................... 1

Owner " Buckskin ................... 2 2

Owner " Kate Hazard............... dis.

Time, 2.44 1 , $242,2.37$.

SEPT. 10TH, 1869.

MANCHESTER PARK, N. H.

SEPT. 10th.-Match $\$-\quad$ Mile heats, best three in five, in harness.

L. W. Wray euters c m Laảy Mallard.......... 1 1 1

Jo€ Fronch " blk m- ............2 22

Time, 3.00, 2.53, 2.513.

SEPT. 11TH, 1869.

ST. LAWRENCE CO., N. Y.

SEPT. 11th.-Purse $\$ 100$, for horses that have never beaten 3.00 . Mile heats, best three in five, in harness.

J. Hinsdale enters br $\mathrm{s}-\ldots \ldots \ldots \ldots \ldots$ \& 1

J. E MeAllister “" br m Lady Mack........2 2 2

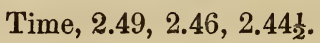

SEPT. 11TH, 1869.

\section{ST. LAWRENCE CO., N. Y.}

S-PT. 11th.-Purse $\$ 225$, for horses that have never beaten 2.35. Mile heats, best three in five, in harness.

A. R Flannagan enters b g Lexington.........3 $5 \begin{array}{lll}1 & 1 & 1\end{array}$

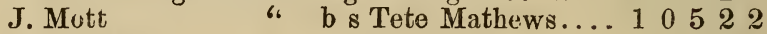

E. L. Harris “ " b g Frank Blair......

R. Dalzell “ "br s Phil Sheridan......5 $4 \begin{array}{llll}3 & 4 & 4\end{array}$

J. H. Phillipps " br g Gov. Morgan......2 02225

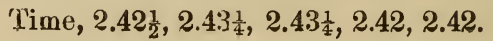


SEPT. $11 \mathrm{TH}, 1869$.

EASTON, Pa.

SEPT. 11th. - Sweepstakes $\$ 75$. Mile heats, best three in five, in harness, for four year olds.

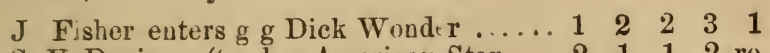

S. V. Davis " b g American Star.....2 11012 ro.

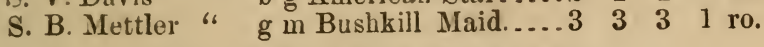

Time, 3.40, 3.38, 3.32, 3.34, 335.

SEPT. 13TH, I869.

NATIONAI COURSE, Washington, D. C.

SEPT. 13th.-Purse $\$ 200$. Mile heats, best three in five, in harness.

O Sanderson enters s m Idaho.............. 1

Win. Lee

6. b m Blanche..............2 2

Time-3:47, 3:06 3: 3:05.

SEPT. 13TH, 1869.

MYSTIC PARK, Mass.

SEPT. 13тн.-Match $\$ 100$. Mile heats, best three in five, in harness.

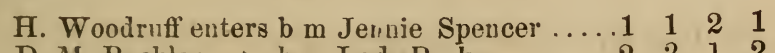

D. M. Bcckler ' b m Lady Peck.........2 2

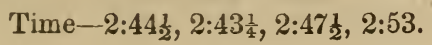

SEPT. $14 \mathrm{TH}, 1869$.

MYSTIC PARK, Mass.

SFPT. 14th.-Purse and stake $\$ 300$. Mile heats, best three in five, in haruess.

W. Woodruff enters b $g$ Centreville........... 1

S. E. Stowell " blkg Kangaroo...........2 \& 2

Time-2.37, 2.36. 2.36.

SEPT. 14TH, 1869.

PROSPECT PARK COURSE, I. I.

SŁPT. 14th.-Purse $\$ 2,000$, for horses that have never beaten 2.25. Mile beats, best three in five, in harness. 
M. Roden enters ch $g$ W. B. Whiteman (formerly

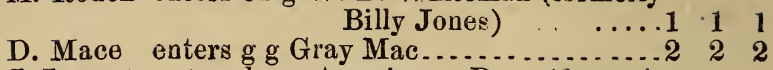

J. Lovatt "b g American Boy (formerly

Henry) .............

Time-2.25 $\frac{1}{4}, 2.24 \frac{3}{4}, 2.24$.

SEPT. 14TH, 1869.

\section{PROSPECT PARK COURSE, I. I.}

SEPT. 14th -Purse $\$ 1,000$, for horses that have never beaten 3.00. Mile heats, best thre in five, in harness.

W. H. Borst
Owner
F. H. Hamilton
Owner
H. Howe
C. Quinten
Owner
H. C. Wooduut
J. Varian

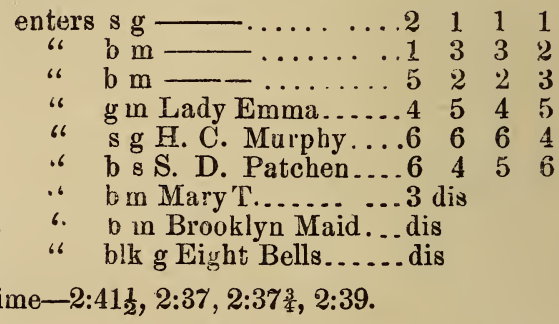

SEPT. $14 \mathrm{TH}, 1869$.

READING, $\mathrm{Pa}$.

SEPT. 14th.-Purse $\$ 300$, for horses that have never beaten 3.ne. Mile heats, best three in five, in wagon.

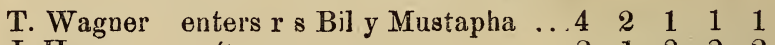
J. Hess
H. Eck
M. Quinn
J Odikirk
R. Dempster
- Conklin
D. Boyer
- Davis
J. Bechtel

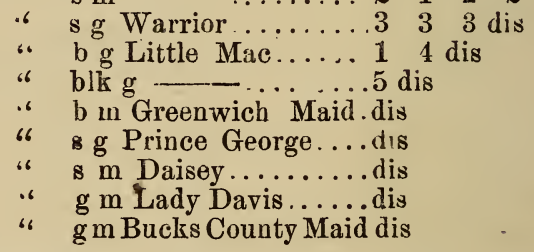

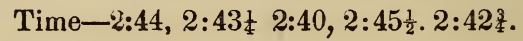

SEPT. 14TH, 1869.

READING, Pa.

SEPT. 14th.-Purse $\$ 800$, for horses that have never beaten 2.30 Mile heats, best three in fire, in harness. 
J. Odikirk enters blk s Patchen Chief.......... 111

A. Goldsmith "“ b m Lady Whitman......... 322

A. Johnson " blk s G. M. Patchen, Jr...2 33

J. Turuer “ b b Victor Patchen......... dis

Time, 2.34군, 2.38. 2.373.

SEP'T. 14 TH, 1869.

HAMMRAMCK COURSE, Detroit, Mich.

SEPT. 14th.-Purse \$-, for horses that have never beaten 2.50. Mile heats, best three in five, in harness.

W. H. Wilson enters b g Strideaway.......... 1

W. Brown " b in Lady Ellwood .......2 22

J. T. Hunter " br g Frank Burroughs...... 3 3 3

D. P. Bissell " b s Lounger............4 dis

Time, 2:35, 2:35, 2:341.

SEPT. $14 \mathrm{TH}, 1869$.

HAMTRAMCK COURSE, Detroit, Mich.

SkPT. 14th.-Purse $\$ 300$, for all horses six years old and under. Mile heats, to wagons.
Wm. Browu
A. P. West
H. MeGregory
H. Chappell
$\begin{array}{cccc}\text { enters b m German Girl. .. ...2 } & 1 & 1 \\ \text { ". blk m Julia Glover...... } & 2 & 2 \\ \text { "“ blk m Cozette ........ } & 3 & 3 \\ \text { " } \quad \text { r s Capt. Tom ......... } & 4 & 4\end{array}$

Time, 2.393 $2.35 \frac{1}{4}, 2.40$.

SEPT. 14TH, 1869.

HAMTRAMCK COURSE, Detroit, Mich

SEPT. 14th.-Purse \$-, for horses that have never beaten 2.29. Mile heats, best three in five, in harness

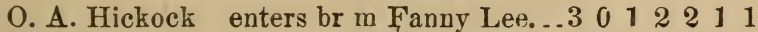

D. P. Bissell " b b Point Bretze..5 $6 \begin{array}{llllll}6 & 1 & 4 & 3 & 2\end{array}$

ๆ. W. Dimmick “ ch s Byron........2 44333124

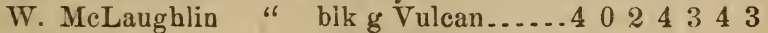

Orwner " blk g India Rubber.1 3446555 dis.

W. Brown “ b g Dick Hilliard..6 $555 \mathrm{dr}$

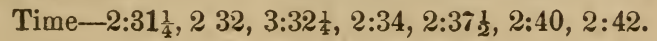


HIT. LAWRENCE CO., N. Y.

SEPT. 14TH, 1869.

SEPT. 14th. - Purse $\$ 100$, for horses that have never started for match, purse or plate. Mile heats, best three in five, in harness.

E. Doran enters b m May Queen...2 111

W. Van Volkenburgh “" b m Miunie.......

H. Nye "* b m Tib.........

Time, 3.041, 3.00, 3.003, 2.59.

\section{SEPT. $15 \mathrm{TH}, 1869$. \\ PATERSON, N. J.}

SEPT. 15th. - Sweepstakes $\$ 200$. Mile heats, best three in five, in harness.

R. B. Galloways enters b s Happy Medium......... 1

J. Y. Diters ". b s Guy Miller............2 dis

J. McKee

J. B. Deau

". b s Honesty.............. dis?

" b s Hambletonian.......... dr

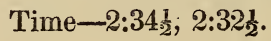

SEPT. $15 \mathrm{TH}, 1869$.

\section{DANBURY PLEASURE PARK,}

SEPT. 15th-Purse $\$ 175$, free for all horses. Mile heats, bist three in five, in harness.
C. Dickerman
R. Mabbit
S. Sniffen

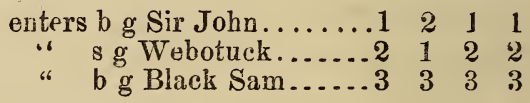

Time-2.44 $\frac{1}{2}, 2.40,2.41,3.01 \frac{1}{2}$

SEPT 15TH, 1869.

\section{UNION COURSE, I. I.}

SEPT. 15th.-Purse and stake $\$ 500$. Mile heats, best three in five to wagon.

H. Casey enters b]k $g$ Black Dan........... 1

Dr. Ogle " b g Red Rover.............. 32

B. Daniels “ b g Young Commodore......4 3 3 3

R. J. Anderson " ch g Willie Kimmy..........2 dis

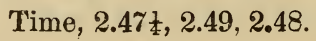


SEPT. 15 TH, 1869.

\section{UINION COURSE, L. I.}

SEPT. 15th-Sweepstakes $\$ 300$. Mile heats, best three in fire.

$\mathrm{R}$ Ogle enters b g Tommy Dodd, (in

harness)... $3 \quad 2 \quad 1 \quad 1 \quad 1$

J. Martin enters b g Rob Roy,(to wagon). $3 \quad 1 \quad 3 \quad 3 \quad 2 \quad 2$

IV. Ferguson '. b $\mathrm{m}$ Sallie, (in harness). $1 \quad 3 \quad 2$ dis

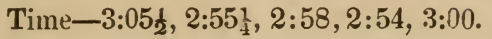

\section{SEPT. 15TH, 1869.}

READING, Pa.

SEPT. 15th.-Purse $\$ 500$, for horses that have never beaten 2.35 . Mile heats, best three in five, in harness.
J. Odikirk
J. Hess
J. Turner
F. Wagoner

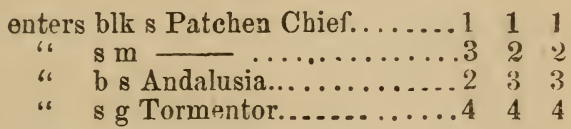

Time, $2.44 \frac{1}{2}, 247,2.40 \frac{1}{2}$.

SEPT. 15TH, 1869.

READING, Pa.

Sept. 15Th.-Purse $\$ 400$, for horses that have never beaten 2.40 . Mile heats, best three in five, in harness.
J. Odikirk
J. Hess
J. Turner
A. De Hart
F Wagoner
R Dempster

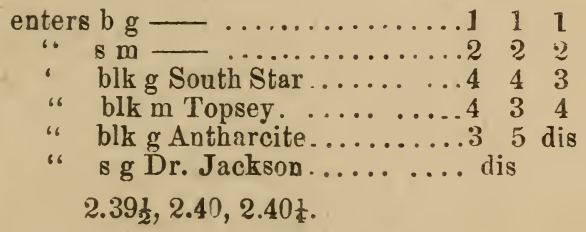

SEPT. 15TH. 1869.

\section{BINGHAMPTON, N. $\mathbf{Y}$.}

SEPT. 15th.-Purse $\$ 300$, for horses that have never trotted better than 3.ก0. Mile heats, best three in five, in harness. 


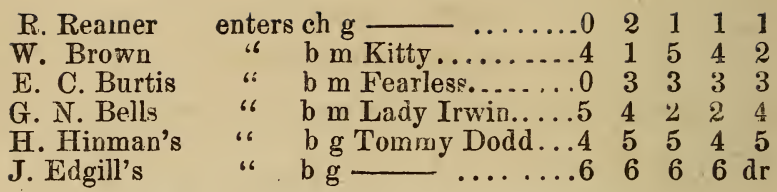

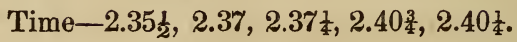

SEPT. 15TH, 1869.

DUTCHESS CO., N. Y.

SEPT. 15th.-Purse $\$-$, for road horses that have never trotted for money. Mile heats, best three in five, to wagon.

R. J. Titus enters $\mathrm{g} m$ - $\ldots \ldots \ldots \ldots$

J. H. Haight " blk g- ...........

Conklin's “

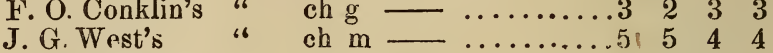

W. H. Seamen " blkg

Time, 2.47, 2.50, 2.46, 2.45 .

SEPT. 15TH,

HAMTRAMCK COURSE, Detroit, MicL.

SEPT 15th -Purse $\$-$ for borses that have never beaten 2.34 Mile heats, best three in five, in harness.

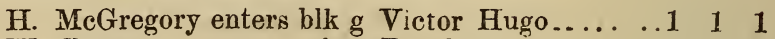

W. Brown " b g Dutchman.........2 2 2

O A. Hickock “ $\quad x \mathrm{~m}$ Edwa................. 3 3

W. Love “

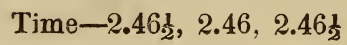

\section{SEPT. 15 TH.}

\section{BAT VIEW PARK, California.}

SEPT 15th.-Purse $\$ 300$ for four year old Trotting Horses. Mile heats, best three in five, in Harness.

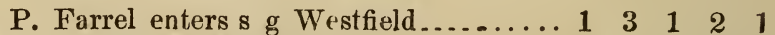

J. L. Eoff “" br g Ajix...............

B. Horn " ch m Breeze..............

Time- $-2.49 \frac{1}{2}, 2.45 \frac{1}{2}, 2.49 \frac{1}{2}, 2.48,2: 47$. 


\section{SEPT. 15TH. \\ POINT BREEZE PARK.}

SFPT. 15th-Purse $\$ 500$. Mile heats, best three in five to wagons Mrs Vosburg' enters s s Vosburgh........... 1 $\begin{array}{llllll}\text { J. Turner } & \text { b } m \text { Lizzie Patchen...... } 2 & 2 & 2\end{array}$

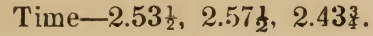

SEPT. $15 \mathrm{TH}$.

\section{ST. LAWRENCE CO., N, Y.}

Sept. 15Th.-Purse $\$ 160$. Mile heats, best three in five, in harness.

S. E. Mathews enters b s Tete Mathews........ 111

R. Dalzell " b g Phil Sheridan ...

A. R. Flannagan " b g Lexington..........dr

Time-2 45, 2.43, 2.45.

SEPT. 15TH.

ST. LAWRENCE CO., N. Y.

SEPT. 15th.-Purse $\$ 100$ for horses that have never beaten 3.00 Mile heats, best three in five, in harness.

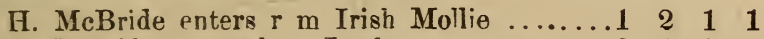

O. Partridge " brg Lookout............

Time-2.58亲, $304 \frac{1}{4}, 2.59 \frac{1}{4}, 259$.

SEPT 16 TH.

ST. LAWRENCE CO., N. Y.

SfePT $16^{\text {th. }}$-Purse $\$ 100$, for Stallions serving in the County. Mile heats. best three in fire, in harness.

J. T. Warner enters $\mathrm{g}$ s Gray Hawk........ 1 1 122

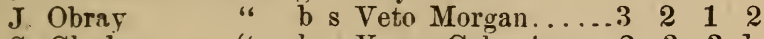

S. Clarke " " b s Young Columbus...2 3 3 $3 d \mathbf{d r}$

Time-3.04 2.54, $249,2.50 \frac{1}{2}$,

SEPT. 16TH.

ST. LAWRENCE CO, N. Y.

SEPT. 16th.-Purse $\$ 250$. Mile heats. best three in five, in harness. Free for all horses. 
A. R. Flannagan enters b g Lexington........ 1 1 1

S. E. Mathews "b b Tete Mathews..... 3 3 2

E. Sargent " ch s Draco Chief........2 2

Time-2.42, 2.42, 2.44.

SEPT. 16TH.

PROSPECT PARK, I. I.

SEPT. 16th.-Purse $\$ 1500$ for horses that bave never beaten 2.30 . Mile heats, best three in five, in harness.

M. Roden enters br g Capt G:ll.......... 1 1

J. Murphy "6 br ma Lady Sears............ 3 ( 32

A. Patterson " br s Manhattan........... 32

F. J. Nodine “" b m Belle of Brooklyn......5 44

D. Pfifer “ br \& Daniel Boone.......2 dis.

Time-2.301, $235 \frac{1}{2}, 2.33$.

SEPT. 16TH, 1869.

READING, Pa.

SEPT. 16th.-Purse $\$ 100$, for horses owned in Berks County. Mile heats, best three in five, to road wagons. Owners to drive.

A. Youngs enters $\mathrm{r}$ s Mustapha........... 11

H. Eppihimers “

E. Ludwig " blk m Black Swallow

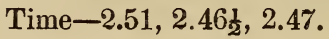

SEPT. $16 \mathrm{TH}, 1869$.

AVON DRIVING PARK, N. Y.

SEPT. 16th.-Purse $\$ 50$, for horses that have uever beaten 3: 1 . Mile heats, best three in five, in harness.

R. Ward enters \& g American Boy .........

F. Shoemaker “ b s Gen. Taylor.........2 0 ¿2

A. Christio "

J. Tubbs “ blk g Raven .......... dis

J. B. Phelps " b m Flora ............... dis

Time, 2.55, 2.58, 2.56, 3.00.

SEPT. 16TH, 1869.

AVON DRIVING PARK, N. $\mathbf{Y}$.

SePt. 16th.-Purse $\$$ - , for horses that have never beaten 2.45 .

Mile heats, best three in five, in harness. 
M. Burvs enters b m Fanny Sheridan........ 1 i 1

F. Shremaker ". b m Lady Wadsworth........ . 22

J. B. Phelps " b m Fiora................

Time-2.53, 2.51, 2.53 .

SEPT. 16TH, 1869.

DUTCHESS CO., N. $\mathbf{Y}$.

SEPт. 16th.-Purse \$-, for road horses. Mile heats, to wagon.

R. F. Ferris enters blk $\mathrm{g}-\ldots \ldots \ldots \ldots \ldots \ldots$.......... 1

i.. Malbitt "6 ch g-_.............. 22

G. Sharpstein "6 b m $\mathrm{m}$

H. Brooks " g g g

Time, $2.41 \frac{1}{2}, 2.41$.

SEPT. 16TH, 1869.

BINGHAMPTON DRIVING PARK, N. Y.

SEPT. 16th.-Purse $\$ 600$, for horses that bave never beaten 2:33. Mile heats, best three in five, in harness.

A. J. Feek enters b m Kitty............ 1 1 $3 \begin{array}{lll}3 & 1\end{array}$

H. Hinman " $\quad$ b g Twang.............

M. Cronk $\quad * \quad \mathrm{~g} g$ Gray John..........

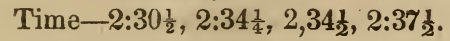

SEPT. 16TH, 1869.

BINGHAMPTON, N. $\mathbf{Y}$.

SEPT 16TH.-Purse $\$ 400$, for horses that have never beaten 2:50. Mile heats, best three in five, in harness.

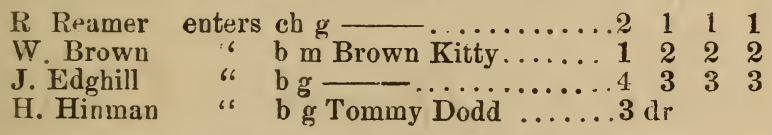

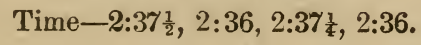

SEPT. 16TH, 1869.

BINGHAMPTON, N. $\mathbf{Y}$.

SkPT. 16th.-Purse $\$ 1,200$. Mile heats, best three in five, in harness. 
H. S. Russell enters b \& Hotspur............ 1 W. H. Saunders " b g Western New York....2 2 S. R. Clark " b m Mountain Maid........ 3 3 3

Time, 2.281 $2.30 \frac{1}{2}, 2,27 \frac{1}{4}$.

SEPT. 16TH, 1869.

HAMTRAMCK COURSE, Detroit.

SEPT. 16th.-Purse $\$ 500$, for horses that have nevor be:ten 2.40. Mile heats, best three in five, in harness.
H. Wilson
A. Rolfe
G. W. Voorhees
W. Brown
O. W. Dimmick

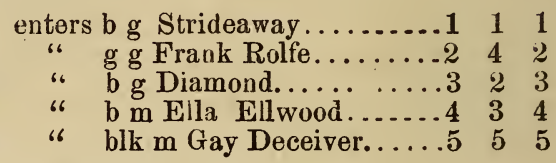

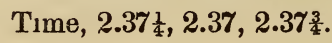

SEPT. 17TH, 1869.

BINGHAIMPTON, N. Y.

SEPT. 17th.-Purse $\$ 500$, for horses that have never beaten 2.37 . Mile heats, best three in five, in harness.
H. Hinman
W Brown
E C. Burtip
enters $\mathrm{b} g$ Twang.............. 1
" b m Brown Kitty.......... 2
“ b m Fearless.............. 3 3 3
Time, $235 \frac{3}{4}, 2.41,2.40 \frac{1}{2}$.

SEPT. 17 1869.

\section{BINGHAMPTON, N. Y.}

SEPT. 17th.-Purse $\$ 4,000$, free for all horses. Mile heats, best three in five, in harness.

B. Doble enters b m Goldsmith Maid........... 1 1 1

B. Daniels “ b m American Girl...........2 22

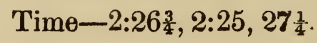

SEPT. 17TH, 1869.

BINGHAMPTON, N. $\mathbf{Y}$.

SePr. 17th. - Purse $\$ 500$, for borses owned in Broome Co. Mile heats, best three in five, in harness. 
J. Bigsby enters b o Bay George.............2 141 M. Cronk " blis s Dr. Kane............ 2 \& 2

R. Wilsou " s s Andrew Jackeon.......... dr

Tine, $2.43,2.42 \frac{1}{2}, 2.42 \frac{9}{4}, 2.438$.

SEPT. 17TH, 1869.

DUTCHESS CO., N. Y.

SEPT. 17th.-Purse \$—. Mile heats, best three in five, to wagons.

D. Mabbitt enters ch $g-\ldots \ldots \ldots \ldots \ldots 1 \quad 1 \quad 1^{*}$

R. Ferris " blkg

G. Sharpstein " bg - ............. ir

Time-2:45, 2.55, 2:57.

SEPT. 17TH, 1869.

DUTCHESS CO., N. Y.

Sxpr. 17th.-Gold Medal for stallions. Mile beats, best three in five, in harness.

Mr. Halstead enters s Burger................ 11

Dr. Barton " s Bull................... \& 2

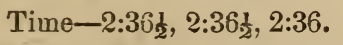

SEPT. 17TH, 1869.

BURIINGTOIN, Vt.

SEPT. 17th-Purse $\$ 250$. Mile heats, best three in five, in harness.

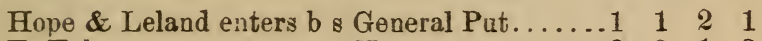

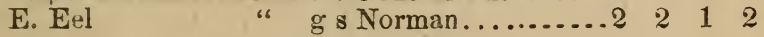

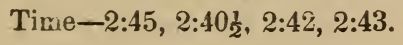

SEPT. 17TH, 1869.

BURLINGTON, Vt.

SEPT. 17th-Purse $\$ 150$. Mile heats, best three ir five, in harness.

$\begin{array}{lllllll}\text { J. Rowell enters br s Billy Bowlegs...1 } & 1 & 2 & 0 & 1 \\ \text { E. H. Gilman “ } & \text { b \& Columbus, Jr. ...2 } & 2 & 1 & 0 & 2\end{array}$

Time-2:44, 2:54, 2:50, 2:49, 2:42. 
SEPT. 17тн, 1869.

BURLINGTON, Vt.

SePr. 17th.-Purse $\$ 175$, free for all horses. Mile heats, best three in five, in harness.

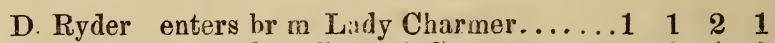

E. Flanuery ". b g General Grant.......2 21

Time-2:42, 2:40, 2:42, 2:43.

SEPT. $17 \mathrm{rH}, 1869$.

\section{OLENTANGY PART, Columbus, Ohio.}

SePt. 17th.-Purse $\$ 1,000$ Mile heats, best three in five, in harness.

A. J. Woodman enters ieorge D............ 1112

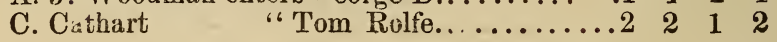

Time, $2.44 \frac{1}{1}, 2.43 \frac{1}{4}, 2.48,2.45$.

SEPT. 17 TH, 1869.

EASTON, Pa.

SEPT. 17th.-Purse $\$ 35$. Mile heats, best three in five, in harness.

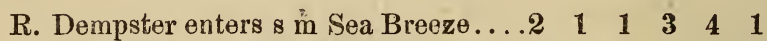

$\begin{array}{llllllllll}\text { D. S. Fields } \quad * & \text { b g Drover......... } & 3 & 3 & 2 & 1 & 1 & 2\end{array}$

$\begin{array}{lllllllllll}\text { A. Judge "} \quad \text { g } & \text { Royal Charles..1 } & 2 & 3 & 2 & 2 & 3\end{array}$

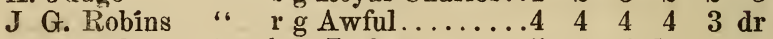

M. Broad "6 b m Lady Frost....5 5 5 $\mathrm{dr}$

Time, 3:0\%, 3:02, 3:08, 3:06 $\frac{1}{2}, 3: 14,3: 0 \%$.

SEPT. 17TH, 1869.

\section{FASTON, Pa.}

SEPT. 17th.-Purse $\$ 500$, free for all horses. Mile heats, best three in five, in bayess.

J. Sollins

J. Hess

enters b m Lady Witman.......... 1

. c m Josephine..................... 22

"6 s m Rosalie............... 33

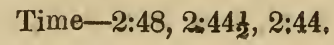




\section{SEPT. 18TH, 1869. \\ AVON DRIVING PARK, N. Y}

SEPT. I8th. -Purse $\$ 35$, for four year olds or under. M le heats, best three in five, in harness:

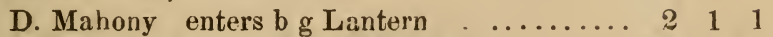

J. Fitzgerald " b m Lady Clay............... 2 dis

P. L. Landon " ch $\mathrm{m}-\ldots . . . \ldots$..... dis

Time, 3.20, 3.67, 30 9.

SEPT. $18 \mathrm{TH}, 1869$.

AVON DRIVING PARK, N. Y.

SEPT. 18th.-Pure $\$ 130$. Mile heats, best three in five, in barness.

$\begin{array}{lcccc}\text { M. Burns } & \text { enters b m Fanny Sheridan........ } & 1 & 1 \\ \text { R. Ward } & \text { 8 g American Boy .......... } & 3 & 3 \\ \text { F. Shoemaker “ } & \text { ch m Lady Wadsworth..... } & 2 & 3 \\ \text { D. Ball } & \text { ". blk s Gen. Taylor.......... } & 4 & 4\end{array}$

Time-2:54, 2:50, 2:53.

SEPT 18TH, 1869.

\section{PROSPECT PARK COURSE, L, I.}

SEPT. 18th.-P'urse $\$ 3,000$, for all horses. Mile heats, best three in five, in harness.

J. D. McMann enters b m Lady Thorn ........ 1

C. Chaplain " b g Geo. Palmer.........2 2

D. Mace " " brs Rhode Island........4 4 4 3

S. MeLaughlin " b b Mountain Boy......... 3 3 4

Time, 2:221 2 , 2:23, 2:22.

SEPT. 18TH, 1869.

\section{PROSPECT PARK COURSE,}

SrPT. 18th -Purse $\$ 1,000$, for horses that have never beaten 2.40. Mile heats, best three in five, in harness

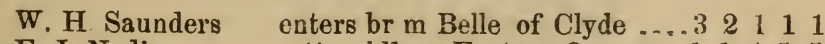

F. J Nodine

J. J. Wheeler

J. Loomis

"6 blk m Eastern Queen... 1114455

" br m Lady Augusta ....4 75532

.6 blk m Soubrette.......2 33223 
J. Malone

H. Bradley

M. Roden enters b s Young Wilkes .....6 63664

" b g Capt. Lsurence.....5 4646

" b g Capt. Smith........75 6 dr

Time, $2.32,2.31 \frac{1}{2}, 2.3 \frac{13}{4}, 2.35 \frac{1}{2}, 2.35 \frac{1}{2}$.

SEPT 18TH, 1869.

\section{HAMTRAMCK COURSE, Detroit.}

SEPT. 18th.--Purse \$2.500. Open to all horses. Mile heats, best three in five, in harness.
O. A. Hickock
O W. Dimmick

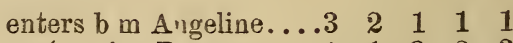
H. S. Compton

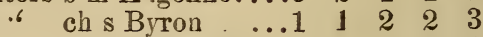
A. Rolfo
" be s Kirkwood...2 $3 \begin{array}{llll}3 & 3 & 3 & 2\end{array}$
"b b g Point Breeze.4 dis

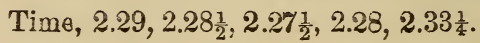

SEPT. 18TH, 1869.

\section{DEXTER PARI, Chicago.}

SEPT. 18ih.-Parse \$- for horses that have never beaten 2.40. Mile heats, best three in five, in harness.
A. Spink
D. B Fisk
enters ch $\mathrm{g}$ Honest Charlie....... I 11
O. B. Diekinson
" ch g Charlie Nellis........ 0 o 00
. br g St. Lawrence....... 0 0 00

Time, 2.41, 2.38, no time.

SEPT. 18TH, 1869.

DEXTER PARK, Chicago.

SEPT. 18th.-Purse \$—. Mile heats, in harness.

J. B. Lyon

J. Stinson

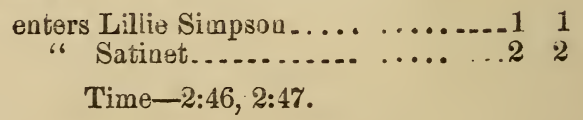

SEPT $18 \mathrm{TH}, 1869$.

DEXTER PARK, Chicago.

SEPT. 18th. Purse $\$$, for horses that have never beaten 2.50 Mile heats. 
J. Stinson enters ch g John (to wagon)........ 0

F. A. Sterens " b g Tom (in harness).......1 2 2

F. H. Keefe " br m Dolly Spanker, (ii

harness) ... $0 \quad 3 \quad 3$

'Time, 2.54, $249,2.53$.

SEPT.. 20TH 1869.

FASHION COURSE, I. I.

SePT. 20th.-Sweepstakes $\$ 300$. Mile heats, vest three in five.

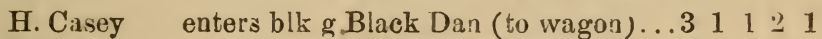

Dr. Ogle “ b g R $\rightarrow d$ Rover (in harness)..... $12221 \%$

R. J. Anderson " ch g Willie Kimmy (in haruess). 23333

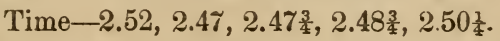

SEPT. 21sT, 1869.

FASHION COURSE, I. I.

SEPT. 21st.-Match $\$ 2,000$. Mile heats, best three in five, in harness.

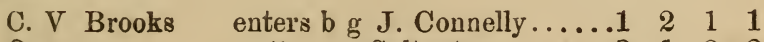

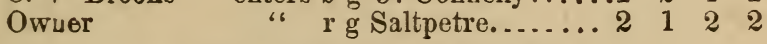

Time, 2:591 $\frac{1}{4}, 2: 47,2: 46,2.45 \frac{1}{4}$.

SEPT. 21ST, 1869.

CITIZENS' PARK, Woonsocket, R. I.

SEPT. 21st.-Purse \$40. Mile heats, best three in five, in harness.

W. S. Briggs

F. B. Wade

W. M. Cameron

R. Chilson

enters $\mathrm{b}$ m Cutriver............ 11

" blk m Lady Gray........3 22

" ch s Cloud.............2

“ br g. St. Lawrence.......4 44

Time, 2.46, 2.45, 2.45 .

SEPT. 22ND, 1869. CITIZENS' PARK, Woonsocket, R. I.

SePT. 22nd.-Purse \$75, for six year old stallions. Mile beats best three in five, in harness.

T. Carpenter enters b!k s Thomas Jefferson...... 1 I 1

W. Cameron " ch s Cloud...............2 2

Time, 2.55, 2.48, 2.40 . 
SEPT. 22ND, 1869.

RIVERSIDE PARK, IMass.

SEPr. 22nd.-Purse $\$ 700$, for horses that have never trotted bctter than 2.34. Mile heat, best thres in five, in harness.

H. A. Hail

G. Knox

J. L. Doty

H. Braciley

W. H. Woodruff

D. Bigley

J. J. Bowen

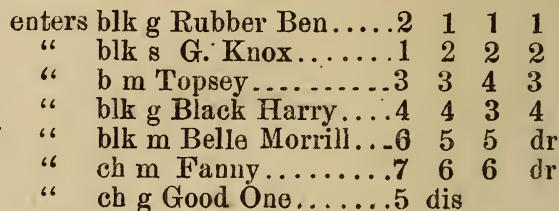

Time, 2.32, 2.33, 2.36, 2.321

SEPT. 22ND. 1800.

SEPT. 22ud-C Irse $\$ 500$ for horses that have never beaten 2.42. Mile heats, best three in five, in harness.
D. Mace
H. Woodruff
S. Røyuo!ds
W. H. Woodruff
enters b g Gen. Sherman....2
“ b m Jennie Spencer...1
“ b m Lady Lightfoot..3
D. M. Beckler
" 8 m Lady Emma.....5
Q

Time, 2:46 $\frac{1}{4}, 2: 42 \frac{1}{2}, 2: 45.2 .45 \frac{1}{2}$.

SEPT. 23RD, 1869.

CITIZENS' PARK, Woonsocket $\mathrm{R}$. I.

SEPT. 23rd.-Purse $\$ 300$. Mule heats, best three in five, in harness.

W. S. Briggs enters blk m Cutwater........ 1 1 $122 \quad 1$

T. Carpenter " b g Johnny Reb..........2 2112

Time-2:37, 2:35 $\frac{1}{2}, 2: 35,2: 40$.

SEPT. 23RD, 1869.

CITIZENS'.PARK, Woonsocket, R. I.

SEPT. 23rd.-Purse $\$ 100$, for horses that have never bөaten 2.40 . Mile heats, best three in five, in harness.

W. S. Briggs enters s $g$ Desmond $-\ldots \ldots \ldots \ldots \ldots 1 \quad 1 \quad 1$

W. Sheldon " r g Roan Jim................. 2

S. A. Bailey " blk g Sleepy David.......... 33

Time-2:44, 2:441 $, 2: 41$. 


\section{1 \\ SEPT. 23RD, 1869. \\ DEXTER PARK, Chicago.}

SкPT. 23rd.-Purse and stake $\$ 800$, for roadsters. Mile heats, best three in five, in harness.

M. Doyle

T. Kelly

B. Wadden

J. Cahill

$\begin{array}{cccccc}\text { enters b m Fanny ............ } & \text { I } & 1 & 2 & 1 \\ \text { " } & \text { blk g Black Ralph.... 1 } & 2 & 2 & 1 & 2 \\ \text { " b m Nelly .............2 } & 3 & 3 & 3 & 3 \\ \text { " ch g Dave Crockett... dis } & & & \end{array}$

Time, $3.04,2.56 \frac{3}{4}, 2.56 \frac{1}{4}, 2.57 \frac{1}{2}, 3,01 \frac{1}{2}$.

SEPT. 23RD, "1869.

DEDHAM, Norfolk County, Mass.

Sept. 23rd.-Purse $\$ 300$. Mile heats, best three in fice, in harness.

C Ricord enters b g Emperor. ............ 111

W. H. Briggs " b g Bay Beauty ......... 2 3 \%

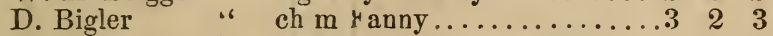

B. Boyce " s g La Havre (from Eli) ...4 4 4 4

C. J. Willis “ $\mathrm{r}_{\mathrm{g}}$ Vermont Boy.........5 $5 \quad 5 \mathrm{dr}$

Time- $-2.39 \frac{1}{2}, 2.38 \frac{3}{4}, 2.49 \frac{1}{2}$.

SEPT. 23RD, 1869.

DEDHAM, Norfolk County, Mass.

SEPT. 23rd.-Purse $\$ 100$, for county horses that nerer trotted for money. Owners to drive. Mile heats, best three in five, in harness.

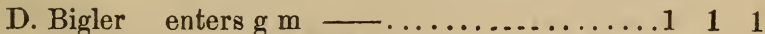

J. E. White " b g Drew..................2 2

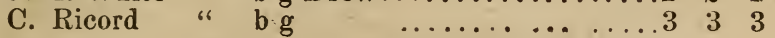

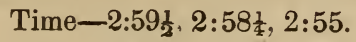

SEPT. 24TH, 1869.

LOWELL RIDING PARK, Mass.

SEPT. 24th.-Match $\$ 100$. Mile heats, best three in five, in barness.

Owner enters Gray Ned................

Owner

Red Leg.................. 1 2 25

Time--2:54, 2:46, 2:50, 2:50. 


\section{SEPT. 25TH, 1869. \\ HARTFORD, Conn.}

SePT. 25th.-Purse $\$$ - Mile heats, best three in five, in harness.

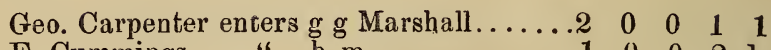

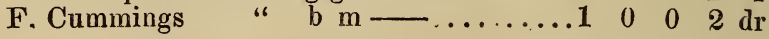

Time, 2.413, 2.42, 2.49, 2.45, 2.52.

SEPT. 25TH, 1869.

HAMPDEN PARK, Springfield, Mass.

SePt. 25Tr.-Purse $\frac{}{6} 100$. Mile heats, best three in five, in harness.

$\begin{array}{llllllll}\text { C. Mosher } \quad \text { enters blk m Black Betty...3 } & 3 & 3 & 1 & 1 & 1\end{array}$

D. C. Pitkin " b $\quad$ g Tom Thumb.....

H. H. Harris " $\quad$ g g Calamity .........

Time, 2.49, 2.47, 2.481 $2.50,2.49,2.49$.

SEPT. 25TH, 1869.

HAMPDEN PARK, Mass.

SEPT. 25th.-Purse $\$ 75$, for horses that have never beaten 2:50. Mile heats, best three in five, in harness.

J. Reed

J. $\mathrm{N}$ Prew

M. H. King

H A. Davis

A Hatch

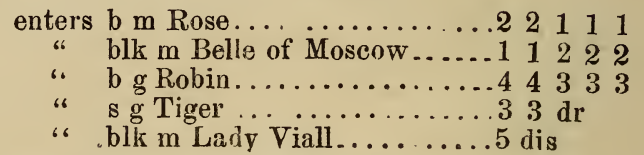

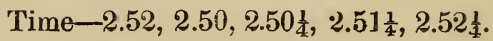

SEPT 25TH, 1869.

\section{RIVERSIDE, PARK MASS.}

SEPT. 25th.-Purse $\$ 1,000$, for horses that have never beaten 2:30. Mile heats, best three in five, in harness.

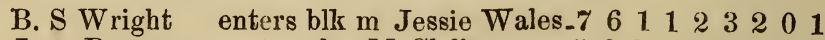

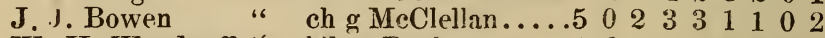

W. H. Woodruff " blk s Darkness....... $3 \begin{array}{lllllllllllllll}3 & 3 & 2 & 1 & 2 & 3 & 3 & 3\end{array}$

J.L. Doty * b m Topsey........6 55564 ro 
G. H. Gilbraith enters blk s G. Knox...... 104045 dis .

D. Mace "w w White Fawn...2464 dis

H. Bradley " blk g Black Walnut_4 dis

Time, 2.31, $2.31,2.34,2.33,2.34,2.32,2.36 \frac{3}{4}, 2.37 \frac{1}{2}, 2.38$.

SEPT. 25TH, 1869.

\section{RIVERSIDE PARK, Mass.}

SEPT. 25th.-Purse $\$ 50$. Mile heats, best three in five.

H. Woodruff enters $\mathrm{gm}$ Lady Lawrence (in harness). 1

D. Bigley " b m Kate, (to wagon).......... dis

G. Howard

" w g Snow Storm............ dis

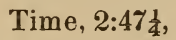

SEPT, 25TH, 1869.

MYRICKSVILIE, Mass.

SePt. 25th.-Purse \$50. Mile beats, best three in five, in harness.

H. Rngers enters $g \mathrm{~g}-\ldots \ldots \ldots 1 \quad 1 \quad 2 \mathrm{dr}$

C. R. Richmond " blk m Cutriver........

Time, 2.33논 $2.35,2.39$.

SEPT. $2 \%$ TH, 1869.

MYSTIC PARK, Mass.

SEPT. 27th.-Match $\$ 3(0$. Mile heats, best three in five,

J. E. Stewart enters b m Jennie Spencer

(in harness)... $122 \quad 1 \quad 1$

Owner

enters s m Lady Peck, (to

$$
\text { wagon)...2 } 1212
$$

Time, 2:421 $\frac{1}{2}, 2: 49,2: 45 \frac{1}{2}, 2: 46$.

SEPT. 28TH, 1369.

MYSTIC PARK, Mass.

SEPT. 28th.-Purse $\$ 500$, for horses that have never beaten 2.5 . Mile heats, best three in five, in harness. 
D. Mace

G. Carpenter

enters br g Drift.................6 31111

J. J. Bowen

"6 ch $\mathrm{m}$ None Such............1 $1222 \%$

W. H. Woodruff "

S. E. Stowell ":

C. Wyman "

S. Hayes

g g Royal John............7 $4 \begin{array}{llll}3 & 3 & 3\end{array}$

cn m Enma...............25 4 dis

br m NoName.............. 65 dis

$\mathrm{g} g-\ldots \ldots \ldots \ldots \ldots . \ldots, 26 \mathrm{dis}$

H. C. Woodruff “" b m Brooklyn Maid........ dis

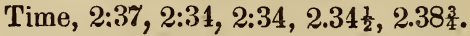

SEPT. 28TH, 1869.

MYSTIC PARK, Mass.

SEPT. 28th.-Purse $\$ 1,000$, for horses that have never beaten 2.34. Mile heats, best three in five, in harness.

\begin{tabular}{|c|c|c|c|c|}
\hline L. Dot & ent & m Topse & & \\
\hline W. S. Briggs & “ & b s Garibaldi....... 8 & 1 & $1 \bar{I} 10$ \\
\hline H. Bradley & "6 & blk g Black Harry....2 & 4 & \\
\hline B. Doble & “ & b g D̊ot. & 10 & \\
\hline W. H. Woodruff & “ & blk m Balle Nurri & 5 & \\
\hline M. Roden & " & br s Manbattan.. & 6 & \\
\hline R. L. Flanders & “ & b g Dew Drop. & 7 & \\
\hline D. Mace & “" & b m Lydia Thompsonio & 8 & \\
\hline S. B. Woodward & “ & sg Artemis W wrd. & 3 & L1) \\
\hline C. N. Howland & " & ch m Lady Carter. & 9 & \\
\hline W. B Smith & " & b g Johnny Reb. & 11 & \\
\hline G. H. Bailey & “ & cola_. & dis & \\
\hline & “" & & dis & \\
\hline
\end{tabular}

Time, 2.32, $234 \frac{1}{4}, 2.34 \frac{1}{4}, 2.33$.

SEPT. 28TH, 1869.

ERIE, Pa.

SEPT. 28th.-Purse $\$ —$, for Erie County horses. Mile heats, best three in five in harness.

J. Childs

J. Wadsworth

W. Colby

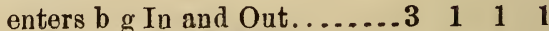

“ blk m Lady Custar....2 32 2 2

Becker \& Co.

" g m Lady Wa isworth. $1 \quad 2 \quad 3 \quad 3$

b g Honest George_..... $55 \quad 5 \quad 5$

" b m Julia.......... . $4 \quad 4 \quad 4 \quad 5$

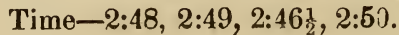


SEPT. 28TH, 1869.

MANCHESTER, NN. H.

SEPT. 23tt.-Purse \$2,500. Mile heats, best three in five, in harness.

C. M. Buchanan enters br s Morrill Prince..3 33111

C. H. Pierce " b g Gen. Hancock...I 2112232

W. H. Moody “ b m Nelly..........2

N. W. hay " ch in Ladv Mallard..54432 ro

H. P. Wingate " " $\mathrm{g} \mathrm{m}$ Cora Belle...... 87543 ro

G. B. Flanders “ blk g Black Prince_.8 88666 ro

J. L. Rodgers “ " b g Plymouth Boy...4 6659 ro

G V. Pickering “. b s Pickering.......7 5 7 dr

Time, $2.47 \frac{1}{2}, 2.47_{\frac{1}{4}}, 2.47,2.46,2.46,2.46$.

SEPT. 28TH, 1869.

MANCHESTER, N. H.

SEPT. 23th.-Purse $\$ 50$. Mile heats, best three in five, in harness.

R. Smith enters b g Shakespeare............. I 11

J. Berry " 8 g Bully Boy...............2 \& 2

Time, 3.18, 3.19, 3.19.

SEPT. 29тн, 1869.

COLD SPRING COURSE, Milwaukee, Wis.

SEPT. 29th.-Purse $\$ 500$, for horses that have never beaten 2.29. Mile heats, best three in five, in harness.

O. A. Hickock enters Fanny Lee............. 1

L. C. Chase "“ India Rubber............2 2

J. Demas “ “ Vulcan..................

Time, 2.34. 2.291 , 2.32.

SEPT. $29 \mathrm{TH}, 1869$.

READING, Pa.

SEPT. 29th.-Pirse $\$ 100$, for horses that have never beaten 3:00. Mile heats, best threg in five, in harness.

L. J. Bertolette enters Diana............. 1

L. Mobr

"Capt. Bill............2 22

Time, 2.40, 2.43, 2.40. 
COID SPRING COURSE, Milwaukee, Wis.

SePt, 29th.-Purse $\$ 500$, for horses that have never beaten 2.34 . Mile heats, best three in five, in harness.

0. A. Hickock enters Edna ............... $3 \begin{array}{lll}3 & 1 & 1\end{array}$

J. Demas

W. Skinner

A. Lowis

" Lottie Clarke.........2 $12 \%$

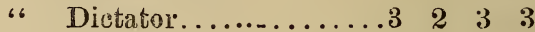

“ Gray Hawk........... dis

Time, 2.38, 2.33! $2.33 \frac{1}{4} ., 2.36$,

SEPT. $29 \mathrm{TH}, 1800$.

READING, Pa.

SEPT 29th.-Purse $\$ 100$, for four year olds, owned in Berks Co. Mile heats, best three in five, in harness.

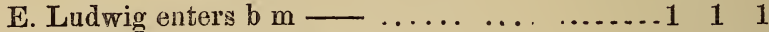

L. F. Fisher " blk $\mathrm{m}-\ldots \ldots \ldots \ldots \ldots \ldots . .22$

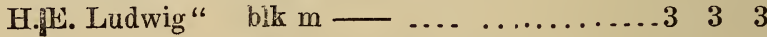

Time, 3.06, 3.01, 3.04.

\section{SEPT. 29TH, 1869. \\ MYSTTC PARK, Mass.}

SEqT. 29th.-Purse $\$ 500$, for horses that have never beaten 2.40 . Mile heats, best three in five, in harness.

F. J. Nodine enter: blk m Eastern Queen, ....... 1 1 1

W. B. Smith " blk s Thomas Jefferson....... 4 4 2

O. M. Shaw “ br g Penobscot Chief....

H. Bradley "6 b g Captain Lawrence....

D. Mace “ b b g Gen. Sherman. ..

S. Reynolds " " b m Lady Lightro ot .........6 6 5 6

B. Daniels " " b g Big Jim..... 5 dis

J. E. Stewart “ b in Jennis Spencer........ dis

Time, 2.37 $\frac{1}{4}, 3.33 \frac{1}{2}, 2.33$.

SEPT. 29TH.

FAMPSHIRE PARIK, Amherst, Mass.

SePT 29th.-Purse $\$ 75$, for all horses. Mile heats, best three in five, in harness.

E. C. Robinson enters br g Gen. Sheridan..... 1 . 11

C. Mosher

" bl m Black Betty.......2 22

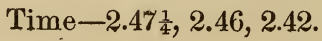




\begin{abstract}
97
SEPT. 29TH.

HAMPSHIRE PARK, Amherst, Mass.

SEPT. 29th.-Purse $\$ 50$, for horses that have never bøiten 3:0".
E. C. Robinson enters b g Young Dexter. $\begin{array}{cccccc}3 & 3 & 1 & 1 & 2 & 1\end{array}$
C. Mosher
T. T. Sisson
" br m Brown Betty..1 23212
" g m Lady Aiken....2 122333

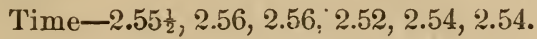
Mile beat, best three in five, in harness.

SEPT. 30TH.

SALEM, N. J.

SEPT. 30th-Purse $\$ 100$, for county horses, mile heats, best three in five, in harness.

J. S. Newell enters b g Stephen A Douglass... 12211

F Pettit " s g Young Star..........2 1222

B. Black "6 b m Polly Ann............ 3333

Time, 2:57, 2:48, 2:51, 2:51

SEPT. 30Th, 1869.

SAIEM, N. J.

SEPT. 30th.-Purse $\$ 100$. Mile heats, best three in five, in harness.

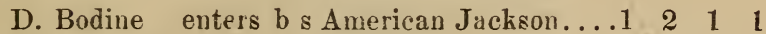

E. W. Brick "

J. H. Locke " g m Lady Locke......... 3 dr

Time-2:46, 2:47, 2:47, 2:47.

SEPT. 30TH.

SALEM, N. J.

Sept. 30th.-Purse $\$ 500$, for horses that have never beaten 2.30. Mile heats, best three in five, in harness.

J. Turner enters b m Fanny Allen............. 1

D. Bodine " b g Victor Patchen.........2 2

Time-2.44, 2.38, 2.41. 


\section{8}

COLD SPRING COURSE, Milwaukee, Wis.

SEPT. 30TH.

SkPт. 30тн.-Purse $\$ 50$, for horses that have never beat: $n$ 2.40. Mile heats, best three in five, in harness.

J. Demas enters Lottie Clarke.........1 11001

E. D. Rood " Minnesota Belle. .......2 22006

S. Crooks " Blonde..............dr

Time-2.403, $2.40 \frac{3}{4}, 2.39 \frac{3}{4}, 2.47$.

SEPT. 30TH.

READING, Pa.

SEPT. 30 TH.-Purse $\$ 250$, for horses that have never beaten 2.32. Mile heats, best three in five, in harmess,

H. Eppihimer ent ers blk m Topsey..... 1222121321

A. Young " $\quad$ rs Mustaph.......2 33321212

A. McRoberts “ br g — .......

D. E. Boyer " s

F. Wanger " s g Tormentor.....4 4 ro

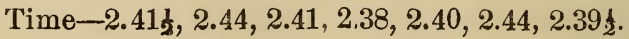

SEPT. 30TH.

READING, Pa.

SEPr. 30tl:-Purse $\$ 50$, for horses that have never beaten 3.10 Mile heats, best three in tive in harness.

D. E. Boyer enters b g Capt. Bill ........2 111

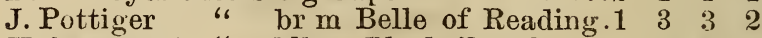

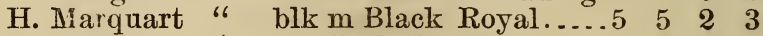

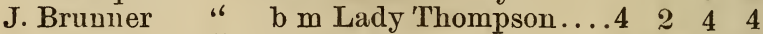

J. Eunis “ b g Bay Billy..........

Time-2.57, 2.56, 2,57, 2.56 .

SEPT. 30TH, 1869.

ABBEY DRIVING PARK, st. Louis, Mo.

SEPT. 30th.-Purse $\$ 500$. Mile heats, best three in five, in harness.

M. Colvin enters b s Pilot Temple.......... 1 1 1

T.P. Roach " s g Morrisey .............2 2

J. MeClure “ b g Trouble.................

T. Best “ $\mathrm{g}$ m Tackey..............

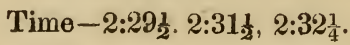


SEPT. 30TH, 1869.

\section{MYSTIC PARK, Mass.}

SEPT. 30Th.-Purso $\$ 1,50$, for horses that have never beaten 2.30. Mile heats. best three in five, in harness.
J. J. Bowen enters ch $g$ Mc Clellan......... 3212121
B. Doble "
L. B Brown " B g Locust.. ..........2988912
W. H. Woodruff “" br \& Mambrine Prince...665 344 ro
W H. Borst “ br g Old Put...........84 4785 ro
H. Bradley " blk g Black Walnut.......9 7566 ro
D. Mace “ s m Henrietta........5 3677 ro
N Carroll “ b g N. B. Palmer........464 43 dis
Owner " b g Dresden... ....78995 dis
F. J. Nodine “ b m Belle of Brooklyn...10 dis
S Emerson “ blk s Darkness..........dis

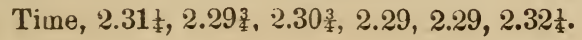

OCT. 1sT, 1859.

MYSTIC PARK, Mass.

OCT. 1st.-Purse $\$ 500$, for horses that have never beaten 3.00 . Mile heats. best three in five, in hariess.

F. W. Burnham enters ch m None Such........ dis

D. Mace $\quad$ br $g$ Drift............

C. Wyman $\quad$ g $g$ g

Time. 2.281 .

OCT. 1st, 1869.

MYSTIC PARK, Mass.

OCT. 1st.-Purse $\$ 3,5 \cap 0$, lor all horses. Mile heats, best three in five in harness.

J. McMinon enters b in Laily Thorn.......... 1 1 1

B. Doble “ b m Goldasmith Maid......... 332

C. Champlain “ br g Geo. Palmer .........2 203

B. Daniels “ "b in American Girl.......... 4 4 4

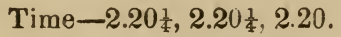

OCT. 1ST, 1869 ,

READING, Pa.

Oct. 1st.-Purs' $\$ 125$ for horses that have never beaten 245 . Mile heats, best three in five, in harness. 


\section{0}

H. K. Becktels euters g m Berks County Maid.2 1211

E. Ludwig "6 blk m Black Swallow...1 2 i 22

H. S. Eckert “ blk g Black Frank......3 3333

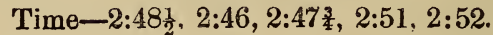

OCT. 1ST, 1869.

READING, Pa.

OCr. 1st.-Purse $\$ 50$, for road horses. Mile beats. best three n five, in harness.

A. Dehart enters blk g Black Hawk.......... 1 1 1

H. Marquart " blk in Black Royal..........2 32

L. G. Fisher " blik m Pet................. 3 \& 3

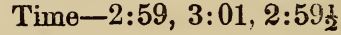

OCT. 1st, 1869.

WYOMING, Penn.

OCT. 1st.-Purse $\$ 50$, for horses that have never beaten 3:00. Mile heats, best three in five, in harness.

P. C. Ecksoth enters blk $m-\ldots \ldots \ldots \ldots 2 \quad 1 \quad 1 \quad 1$

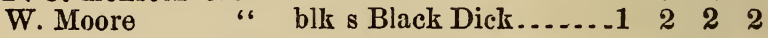

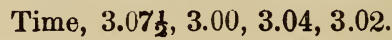

OCT. 1sT, 1869.

COLD SPRING COURSE, Milwaukee, Wis.

Ост. 1st.-Purse $\$ 800$, for all horses. Mile heats, best three in five, in harness.

O. A. Hickock enters Angeline............. $122 \quad 1 \quad 1$

S. Anderson

J. Demas

A. Lewis

" Silas Rich........... $4 \begin{array}{llll}4 & 1 & 2 & 2\end{array}$

“ Vulcan.............2 $4 \begin{array}{lll}4 & 4 & 3\end{array}$

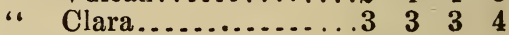

Time, $2.29,2.29 \frac{1}{2}, 2.28 \frac{1}{2}, 2.32 \frac{1}{4}$.

OCT. 2ND, 1869.

FASHION COURSE, L. I.

OCT. 2nd-Sweepstakes $\$ 800$. Mile beats, best three in tive, in harness. 
Dr. Ogle

J. Martin

H. Casey

R. J. Anderson

enters b g Red Rover........1201 201 dis

" g s Gray Messenger....2 10202 dis

“ blk $g$ Black Dan........3 433 dis

" $8 \mathrm{~g}$ Willie Kimmy.....4 3444 dis

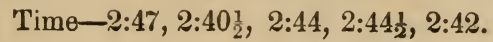

OCT. $5 \mathrm{TH}, 1869$.

NARRAGANSETT PARK, R. I.

OCT. 5th.-Purs9 $\$ 500$, for horses that have never beaten 3:00. Mile heats, best three in five, in harness.

H. Rogers enters b g Butcher Boy........... 1 1 1

Dr. Ogle " $\quad$ b g

E. K. Russell " s m Nelly ............2 dis.

H. Taylor “ b s Nabob..................dis.

W. Baine "6 b g

Time, 2:36, 2:36, 2:39ł.

OCT. 6TH, 1869.

NARRAGANSETT PARK, R. I.

OCr. 6th.-Purse $\$ 1,000$, for horses that have never beaten 2:45 Mile heats, best three in five, in harness.

D. Mace enters br g Drift (formerly Norwood) $1 \quad 1 \quad 1$ F. W. Burnham " s g None Such ........... 4 4 2

H. Rogers

Wu Saunders “"

E. K Russell

b g Ligntfoot

$\begin{array}{lll}2 & 3\end{array}$

“ b m Belle of Clyde............. $3 \quad 3 \quad 4$

$\mathrm{s}$ m Nellie...............5 dis.

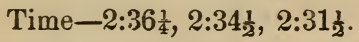

OCT. 6TH, 1869.

NAKRAGANSEIT PARK, R. I.

OcT $6:$ th.-Purse $\$ 2,000$, for horses that bave never beaten $2: 30$ Mile heats, best three in five, in harness.

J. H. Harbeck, Jr. enters g $g$ Surprise........2 11220311

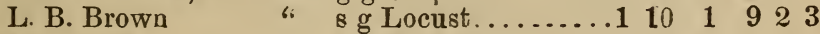

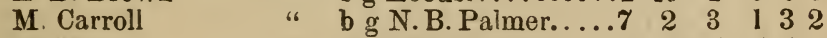

D. Mace

W. H. Burst

O. L. Marshall

w $\mathrm{g}$ White Fawn.....6 6 6 55444 ro

“ br g Old Put........10 $354 \quad 75$ ro

" b $\mathrm{m}$ Belle of Brook-

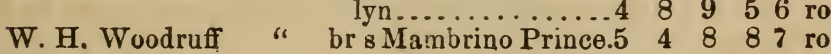


B. Mace

J. Raftus

D. Pfifer

W. S. Briggs

J. H. Gilbraith

enters $b \mathrm{~g}$ Twang $\ldots \ldots \ldots \ldots 3 \quad 7 \quad 6 \quad 2 \quad d r$

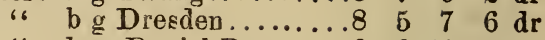

" br's Daniel Boone....9 $91010 \mathrm{dr}$

" b s Garibaldi......... dis.

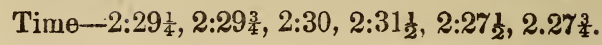

OCT. 6TH, 1869.

WATERTOWN PARK, N. $Y$.

OCT. 6th.-Purse $\$ 100$, for horses that have never beaten 3:00. Mile heats, best three in five, in harnes?.

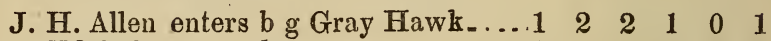

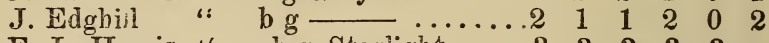

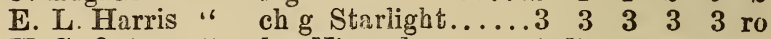

H.C Oatman" b g Nimrod.......4 4 dis

G. L. White " ch $\mathrm{m}-\ldots . .$. . dr

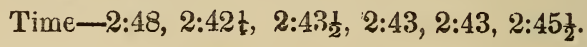

OCT. $6 \mathrm{TH}, 1869$.

IFXINGTON, IXy.

Ocr. 6th.-Purse $\$ 150$. Milo heats, best three in five, in harness.

J. W. Conley " enters b s Wild Wagoner....... 1 1 1

T. Elwood " b m Ella Elwood......... 2

Time, $2.53 \frac{1}{2}, 2.46 \frac{3}{4}, 2.44 \frac{1}{2}$.

OCT. 7TH, 1869.

INARRAGANSFTT PARK, R. I.

OCT. 7th.-Purs $\$ 1,000$, for horses that have never beaten 2:40. Mile heats, best three in five, in harness.

D. Mace enters br go Drift, (formerly Nor.

O. L. Marshall

........3 2125

O. M. Shaw " b s Penobscot Chief.......8 66642

W. H. Saunders “ b m Belle of Clyde........6 77533

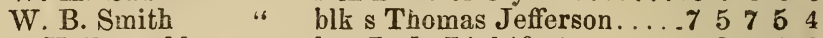

S. K. Reynolds " b b Lady Lightfoot........48 $8+66$

H. Rogers " b g Lightfoot............... $333 \mathrm{dr}$

J. Loomis “ blk m Soubrette............14 dis

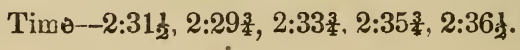




\section{3}

OCT. 7TH, 1869.

\section{NARRAGANSETT PARK, R. I.}

Ocr. 7th.-Purse $\$ 2,000$, for borses that have never beaten $2: 33$. Mile heats, best three in fire, in harness.
B. Mace
enters b
b g Twarg...........125 12121
J. L. Doty
b m Topsey.............454612
J. H. Gilbreth “ blk s G. Krox.........21128 dis
B. Doble
D. Mace $8 \mathrm{~g}$ Dut $\ldots \ldots \ldots \ldots \ldots, 87873$ ro
J.H. Harbeck, jr" H. A. Hall "
S. B. Woodard "“ ch g Artemus Ward..... 76 7 $\mathrm{dr}$
W. S. Briggs “ b s Garibaldi............ dis

Time-2.28市, $2.26 \frac{3}{4}, 2.29 \frac{1}{2}, 2.31,2.31 \frac{1}{2}, 2.31 \frac{1}{2}$.

OปT. 7TH, 1869.

\section{DOYLESTOWN, Penn,}

Ocr. 7th.-Purse $\$ 600$, for all horses. Mile heats, best three in five, in harness.

M. Goodwin

A. R. Smith

J. Wart

E. K. Conklin

enters ch g Harry D........... 1

" b m Lady Whitman.......4 2 2 2

“ b \& Andalusia........... 3 4 3

" $\quad$ s 8 American Star.......2 34

Time, $2.37 \frac{3}{4}, 2.38,2.37 \frac{1}{4}$.

\section{OĈ̀T. 7Тн, 1869.}

\section{WATERTOWN PARK, N. Y.}

OcT. 7th.--Purse $\$ 200$, for horses that have never beaten $2: 40$. Mil: heats, best three in five, in harness.
H. A Wbite
enter's $g \mathrm{~g}$ Jack Uraper.....1 2 J 2221
S. V. York
R. P White
" ch \& Shotwell......2 3211 \%
E. C. White
". b m
" ch m Fanny Fern....343 3 dir

Time--2:41, 2:37, 2:391 2 , 2:42, 2:42, 2:46.

OCT. 8TH, 1869.

SAN JOSE, California.

Oct. 8th.-Purse $\$ 250$, for all horses. Mile heats, best three in five. 
Owner enters May Queen (to wagon).......... $1 \quad 1 \quad 1$

Owner " Lady Dooley (in harness)........ 34 3

Owner ". Regulator (in harness)..........2 $2 \quad 3$

Owner " Princess (in harness)............ 44

Time, $2.33 \frac{1}{2}, 2,35,2.36$.

OCT. 8TH, 1869.

NAZARETH, Pa.

OcT. 8th.-Purse $\$ 100$. Mile heats, best three in five, in harness

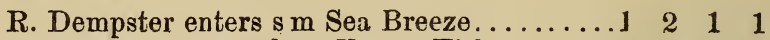

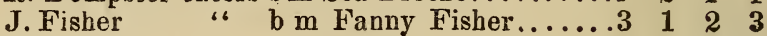

H. C. Eck " s g Warrior...........2 33 3 2

. Time, 2.55, 2.52, 2.48, 2.52 .

OCT. 8TH, 1869.

RIVERHEAD, I. I.

OCT. 8th.-Purse $\$ 50$, for all horses. Mile heats, best three in five, in harness.

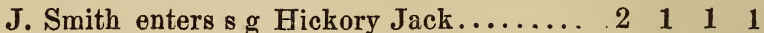

J. Fisher " blk m Lady Sherman.........

M. Brush " br m Tiney................ $3303 \mathrm{dr}$

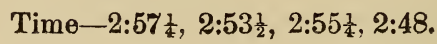

OCT. 8TH, 1869.

\section{RIVERHEAD, I. I.}

OCT. 8th.-Purse $\$ 50$, for horses that have never beaten 3.00. Mile heats, best three in five, to wagon.

G. Hawkins enters b g Ned Buntline ......... 11

D. H. Griffen "6 blk g

Time-3:023, 2:56, 3:10.

OCT 8тH, 1869.

NORTHAMPTON PARK, Mass.

OCr. 8th.-Purse $\$ 250$, for all horses. Mile heats, best three in five, in harness. 
E. C. Robinson enters b g Emperor.......... 11

W. Elliot ". br m No Name... ......2 2

C. Mosher “ blk m Black Betty..... . 33 3 3

Time, 2:43\$, 2:41. 2:37\%.

OCT. 8тн. 1869.

NORTHAMPTON PARK, Mass.

OCT. 8th.-Purse $\$ 150$, for horses that have never beaten 2:40. Mile heats, best three in five, in harness.

E C. Robinson enters g $\mathrm{m}-\ldots \ldots \ldots 1$ 2 11

Wm Elliot ": b g Tom Thumb.......

H. A. Cook " "bik g -

W. H. King " ch g Springfield Boy....3 4 dis

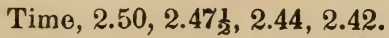

OCT. 8TH, 1869.

DOYLESTOWN, Penn.

OCr. 8th.-Purse $\$ 70$, for horses owned in the county. Mile heats, best three in five, in harness.

C. S. Atkinson enters Lady Douglas.........1 1

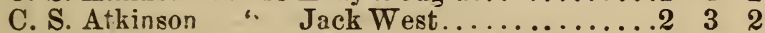

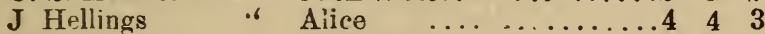

E. K Conklin " Prince George............... 24

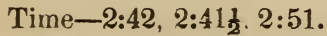

OCT. 8TH, 1869.

DOYLESTOWN, Penn.

Ocт. Sth.-Purse $\$ 70$, for stallions. Mile heats, best $\mathrm{t}$ hre in five. in harness.

F. K. Conklin enters American Star .......... 11

E. Hoffman is Ben D. Patchen ............ 2

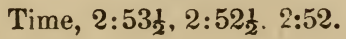

OCT. $81 \mathrm{r}$.

NARRAGANSETT PARK, R. I.

Oct. 8th.-Purse $\$ 1000$, for horses that bave never beaten 2:50. Mile beats, best three in five, in harness. 
F. W. Burnham enters s m None Such....... 1 1 1

Dr. Ogle

H. Taylor

" br h-

" $\mathrm{w} g$ Snow Ball........ dis

Time-2.36 $\frac{3}{4}, 2.38 \frac{3}{4}, 2.33$.

OCT. 8TH.

NARRAGANSETIS PARK, R. I.

OCT. 8th.-Purse $\$ 5,000$, for all horses. Mile heats, best three in five, in harness.

J. D. McMann enters b m Lady Thorn.

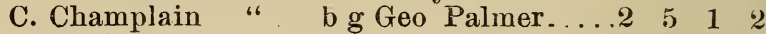

$\begin{array}{lllllll}B & \text { Doble } & \text { " b m Goldsmith Maid } 4 & 2 & 2 & 3\end{array}$

D. Mace " " b m Lucy...........

B. Daniels " " b m American Girl..3 43 dis.

Time-2.193 $, 2.18 \frac{1}{4}, 2.19 \frac{1}{4}, 2.21$.

OCT. 8TH.

WATERTOW N PARK, N. Y.

OcT. 8th.-Purse $\$ 150$, for horses that have never beaten 2:50. Mile heats, best three in five, in harness.

R. P. White enters $\mathrm{b} \mathrm{m}-\ldots \ldots \ldots \ldots \ldots 1$

$\begin{array}{lllllll}\text { J. Edghill } & \text { " b g } & \ldots \ldots \ldots \ldots & \ldots 2 & 2 & 2\end{array}$

E. L. Harris " $\quad$ br s D. M. Rice.......... 33

J. H. Allen “" g s Gray Hawk.........dr

H. B Griffen “ br g Brown Dick .......dr

Time-2.45, 2.47, 2.51.

OCT. 9TH.

FORD'S PARK, Denver, Col.

Ocr. 9th.-Purse and Stake $\$ 250$. Mile heats, best three in five, in harness.

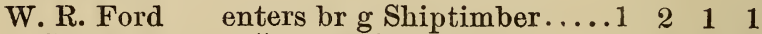

J. Croker . " b g Rainbow.......

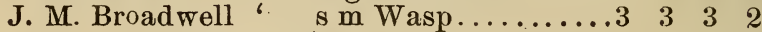

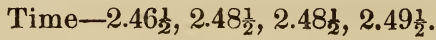

OCT. 9тн.

MIDDLESEX, Mass.

Oct 9th.-Purse $\$ 200$. Mile heats, best three in five, in harness. 
S. Hayes enters blk m Lady Wilkes..... 33 3 11 1 1

S. C. Witcher " s g Romeo.............

A. W. Wooster " b g Billy Button....... $2: 22$

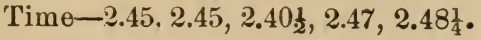

OCT. 9тн.

MIDDLESEX, Mass.

Oct. 9th.-Purse \$170. Mile heats, best three in five, in barness.

R. L. Flanders enters b s Lexington.......... 1

J. S. Lyons " " b m Cambridge Belle...2 2 2

S. Hayes

b $\mathrm{g}$

$\operatorname{Tim} \theta-2.46 \frac{1}{2}, 2.51,2.52$.

OCT. 9TH.

MIDDLESEX, Mass.

Oct. 9th.-Purse \$250. Mile heats, best three in five, in harness.

A. W. Wooster enters ch g McClellan......... 11

J. S. Lyons " blk g Black Walnut ...2 2

R. L. Flauders " b g Dew Drop.......... 3 3 3

Time-2.37, $2.35 \frac{1}{2}, 2.36$.

OCT. 11TH.

FORD'S PARK, Denver Col.

Oct. 11 th.-Purse and Stake \$250. Mile heats, best three in five, in harness.

J. Croker enters blk g Tobe........... 1121

W. R. Ford " b g Col. Webster.

C. R. Ward " blkg Ghost...........

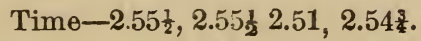

OCT. 13тн.

BUCKEYE COURSE, Cincinnati, O."

Ocr. 13th. - Purse $\$ 400$, for horses that have never beaten 3:00. Mile heats, best three in five, in harness. 


\section{8}

T. P. Roach enters b g Matthew Smith.....3 31111

C. Leggett " s m Annie Hunter......1 12223

A. Kinkard " b g Bay Jim (formerly Mike McCool)....................... 22332

Time-2.37 $, 2.40 \frac{1}{4}, 2.38 \frac{1}{2}, 2.41 \frac{1}{2}, 2.39$.

OCT. 13 TH.

BUCKFYE COURSE, Cincinnati, 0

Ocr. 13th.-Purse $\$ 600$, for all horses. Nile heats, best three in five, in harness.

T. P. Roach enters ch g Morrisey..........2 1121

T. T. Oliver " $\quad \mathrm{b} \mathrm{m}$ Mollie...........

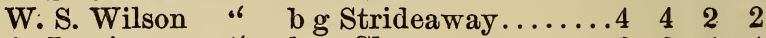

A. Lowis " b b m Clara.........

Time-2.32, 2.291 $2.30,2.31 \frac{3}{4}$.

OCT. 13TH.

DUBUQUE DRIVING PARK, IOwa.

OcT. 13th.-Purse $\$ 100$, for horses that have never beaten 3:00. Mile heats, best three in five, in harness.

G. Clarke enters b g Young Princeton.......... 2

C. Myer " b g Pat Malloy .............. dis.

Time-2.38 $\frac{1}{4}, 2.35 \frac{1}{2}$,

OCT. 13'TI, 1869.

\section{DUBUQUE DRIVING PARK, IOwa.}

Ocr. 13th.-Purse $\$ 175$, for horses that have never beaten 2:40. Mile heats, best three in five, in harness.
D. Hammer
W. W. Hamilton
J. Boyle
G. Nolson
'T. Pierson
A. Jones
enters blk m Jennie Day......... 1 1 1
" b g Billy Hamilton........3 22
". s m Champion Maid.....2 33
- b s W S. King.........5 54
، b g Billy Chandler... ...4 445
“ $g \mathrm{~m}$ Nellie Gray..........6 6 dis.

Time, $2.42 \frac{1}{4}, 2.45 \frac{1}{2}, 2.44 \frac{1}{2}$.

OCT. 13тн, 1869.

DEXTYR PARK, Chicago.

OCr. 13th.-Purse $\$ 1,000$, for all horses. Mile heats, best three in five, in harness. 
M. Colvin

H. Graves

O. H. Hickosk

W. MeLaughlin enters b s Pilot Temple.... ....1 111

"، b g Jim Rockey........2 2 2

“ “ b m Angeline...........4 4 4 3

“ $8 \mathrm{~g}$ Silas Rich..........3 $3 \quad 4$

Time-2:29, 2:291, 2:30.

OCT. 14TH, 1869

\section{DEXTER PARK, Chicago.}

OCr. 14th.-Purse $\$ 300$, for horses that have never beaten $2: 34$. Mile heats, best three in five, in harness.

J. Demas enters blk m Lottie Clark............ 1

M. Colvin " du m Naomi ................2 2

F. Haley "* $\mathrm{g} g$ Sea Foam............... 33

Time-2:391, 2:34, 2:35.

OCT. $14 \mathrm{TH}, 1869$.

\section{BUCKEYE COURSE, Cincinnati, 0.}

Ocr. 14th.-Purse $\$ 400$, for horses that have never beaten 2.48 . Mile heats, best three in five, in harness.

T. P. Roach enters b g Mathew Smith.......2 111

C. Leggitt “ $8 \mathrm{~m}$ Annie Hunter.........

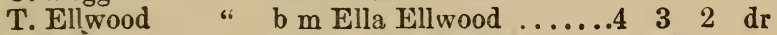

J, Miller

" b s John Esdall..........3 dis.

C. G. Somers “ b m Katie.............5 dis.

Time, $2.33 \frac{1}{2}, 2.36 \frac{1}{2}, 2.36 \frac{3}{4}, 2.45 \frac{1}{2}$

OCT. $14 \mathrm{TH}, 1869$.

BUCKEYE COURSE, Cincinnati, 0 .

OCT. 14th.-Purse $\$ 500$, for horses that have never beaten 2.35 . Mile beats, best three in five, in harness.

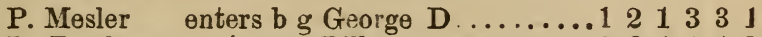

L. Dunham "6 g g Billy.............2 6441112

Fritz \& Roher “ b s Tom Rolfe.......... $6 \begin{array}{lllllll} & 1 & 3 & 4 & 4 & 3\end{array}$

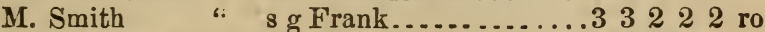

J. B. Loder " $\mathrm{gm} \mathrm{Lou............455} \mathrm{dr}$

J. Miller " ch m Miller's Favorite...5 4 dis,

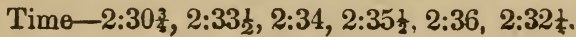


OCT. 14TH, 1869

WAVERIY PARK, IN. J.

OCT 14th.-Match $\$ 100$. Mile heats, best three in five, in harness.

Mr. Merritt enters blk g Knox ............2 1141

W. Gordon " s g Boston Boy............ 2 ? 2

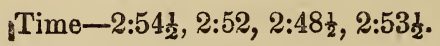

OCT. $14 \mathrm{TH}, 1869$.

VERNON, Orange County, N. Y.

OC1. 14th.-Purse $\$-$, for stallions. Mile heats, in harness.

J. Bertholf enters b s Shelly's Clay............. 1

- Drew " b s Jersey Boy...............2 2

Time, 2:55, 2:52.

- OCT, $14 \mathrm{TH}, 1869$.

DUBUQUE DRIVING PARK, IOwa.

OCT 14th.-Match $\$ 300$. Mile heats, in harness.

H. Clarke enters blk $m$ Black Kate...........1 1

A. Jones "s $\mathrm{m}$ Wild Rose..................2 2

J. Coddington " s s Comet .................. dis

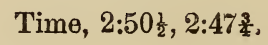

OCT. $14 \mathrm{TH}, 1869$.

DUBUQUE DRIVING PARK, IOwa.

Ост. 14th.-Purse $\$ 250$, for horses that have never beaten 2:35. Mile heats, best three in five, in harness.

J. C. Simpson enters br m Abatross........... 1 1 1

S. D Williams " s s Boone..................

$\begin{array}{llll}\text { J. Boyle } \quad \text { " b g Ed. Price.............. } & 3 & 3\end{array}$

R. Jackson "

Time, $2.42 \frac{1}{2}, 2.42,2.42 \frac{1}{2}$.

OCT. 14TH, 1869.

JAMESTOWN, N. $\mathbf{Y}$.

Oct. 14th.-Purses $\$ 100$. Mile heats, best three in five, in haruess. 


\section{1}

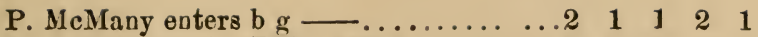

R. J. Warner " Billy Paver...........

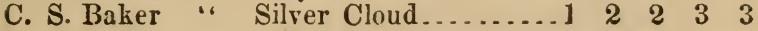

Time-2:45, 2:473, 2:50, 2:49, 2:50.

OCT. 15TH, 1869.

MARIETTA, Ohio.

OCT, 15th.-Purse \$140, for all horses. Mile heats, best three in five, in harness

J. W. Conley enters b s Wild Wadoner.....2 $14 \quad 1 \quad 1 \quad 1$

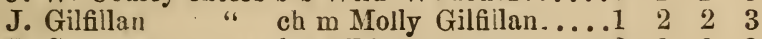

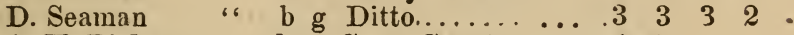

A. H. Ricker " br g Gen. Grant.......4 ais

Time, 2.44눈, 2.40, 2.40, 2.401.

OCT. 15TH, 1869.

BELVIDERE, N. J.

Ocr. 15th.-Purse $\$ 175$. Mile heats, best three in five, in haruess.

J. Fisher enters s g Hiram.............. 1

S. B. Mettler " b g Bill Jackson............2 2

R. Dempster " s m Sea Breezo............ 333

R. Dempster " br m Greenwich Maid....... 5 5 4

J H. Robbins " br'm Flora............... 5 5 4 5

Time, $2: 47 \frac{1}{2}, 2: 47 \frac{1}{2}, 2: 48$.

\section{OCT. 15TH, 1869. \\ BUCKEYE COURSE, Cincinnati, Ohio,}

OCT. 15th.-Purse $\$ 400$, for horses that have never beaten 2.40 . Mile heats, best three in five in harness.
J. Miller enters b g Dick Jamison.2 51144411
R. Strader \& $\mathbf{C}_{0}$.
J. B. Loder
J. T. Biggs
" ch in Lucy........3 42211122
“. r g Lew Sayres.....1 $1150 \begin{array}{lllll}5 & 3 & 3 & 3\end{array}$
T. J. Ellwood
" b g Dart........4332 3 ro
" b m Ella Ellwood..5 $24 \mathrm{r}$ off

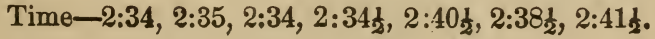


OCT. $15 \mathrm{TH}, 1869$.

\section{DUBUQUE DRIVING PARK, IOwa,}

Oct. 15th.-Purse $\$ 450$, for all horses. Mile heats, best three in five, in harness.

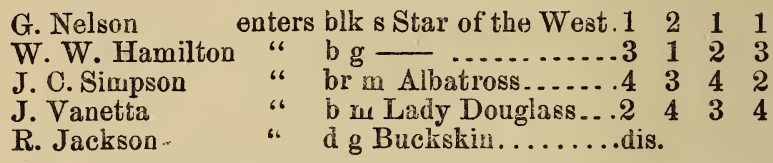

Time, 2.333, 2.37, 2.34, 2.32.

OCT. 15TH, 1869.

DUBUQUE DRIVING PARK, IOWa.

Ocr. 15th.-Purse $\$ 125$, for horses that have never beaten 2.45 . Mile heats, best three in five, in harness.

G. Clarke enters b g Young Princeton.......... 1

F. Bethel "d d gocket.................. 2

Time-2:37, 2:37, 2:403.

OCT. 15тн, 1869.

PITYSFIELD, III.

OCr. 15th.-Sweepstakes $\$$ - Mile heats, in harness.

D. Bates enters d $g$ Buckskin............. 11

C. M. Dunlop “ br m Betty.................

J. M. Blain “ br s Shab................. $33 \mathbf{3}^{4}$

F. Jones “ $\quad$ s g Sherman.............. 44

Time-2:56, 2:54, 2:51.

OCT. $16 \mathrm{TH}, 1869$.

DUBUQUE DRIVING PARK, IOWa.

OCT. 16th.-Match $\$ 200$. Mile heats, best three in five, in barness.

T. Robinson enters $b$ s Iron Duke........... 1

J. Coddington " s s Comet............... 2 dis,

Time-2:531, 2:56, 2:57. 
OCT. $16 \mathrm{TH}, 1869$.

\section{BUCKEYE COURSE, Cincinnati, 0 .}

Ocr. 16th.-Purse $\$ 500$, for all horses, go as they please. Mile heats, best three in five.

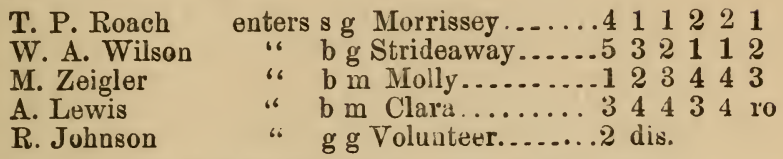

Time, 2.303 $2.30,2.28 \frac{3}{4} .2 .28 \frac{1}{2}, 2.31 \frac{3}{4}, 2.32$.

OCT. $16 \mathrm{TH}, 1869$.

GLENWOOD, Iowa.

Ocr. 16th.-Purse $\$ 100$. Mile heats, in harness.

Mr. Montgomery enters blk s Blackbird........... 1

Mr. Betts

Owner

Mr. Sidners

Mr. Demick

Mr. Bordeno

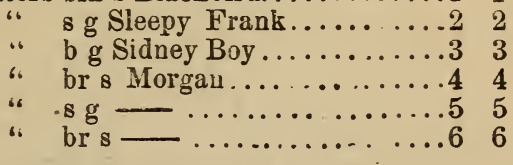

Time-2:50, 2:51.

OCT. 16TH, 1869.

DEXTER PARK, Chicago, III.

Ocr. 16th.-Purse $\$ 1,500$, for all horses. Mile heats, best three in five, in harness.

J. Rockey enters b g Jim Rockey .......... 1

- M. Laughlin “ $8 \mathrm{~g}$ Silas Rich..............2 2 \%

M. Colvin " b s Pilot Temple............dis.

O. A. Hickock “ b m Angeline. .............. dis.

Time-2:321 $\frac{1}{2}, 2: 31,2: 28$.

OCT. $19 \mathrm{TH}, 1869$.

MYSTIC PARK, MIass.

OcT. 19th.--Purse $\$ 100$, for horses that have never beaten 2.50 . Mile heats, best three in five, in harness. 


\section{4}

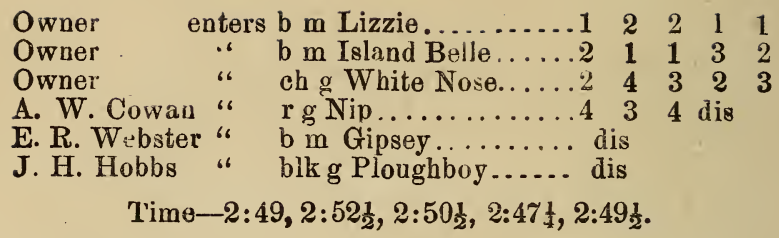

OCT. 19TH, 1869.

MYSTIC PARK, Mass.

OCT. 19th.-Purse $\$ 100$, for all horses. Mile haats, best three in five, in harness.

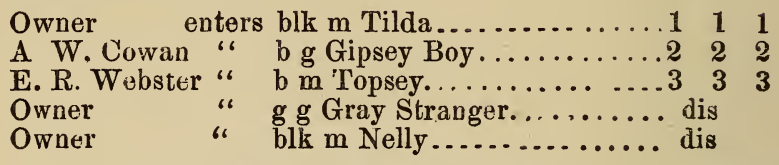

Time, $237 \frac{1}{2}, 2.41 \frac{3}{4}, 2.41 \frac{1}{2}$.

OCT. 19 TH, 1869.

\section{HARTFORD, Conn.}

Ocr. 19th.-Purse $\$ 700$, for all horses that have never beaten 2.38. Mile heats, best three in five, in harness.
W. B. Smith
D. Bigley
J. Woodruff
Owner
M. H. Griffen
enters blk s Thomas Jefferson_...1 11
" ch m Fanny...... .....3 4 4 2
“ b g. Listener...........4 2
“ g g Marshall...........2 30.
" b g Gen. Serrell.........5 55

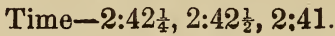

OCT. 19 TH, 1869.

HARTFORD, Conn.

OcT. 19th.-Purse $\$ 400$, for horses that have never beaten 2.50 . Mile heats, best three in five, in harness.

H. B. Goodale
G. P, Carpenter
F. B. Cummings
Owner
M. H. King
W. W. Cameron

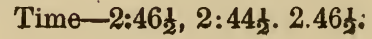

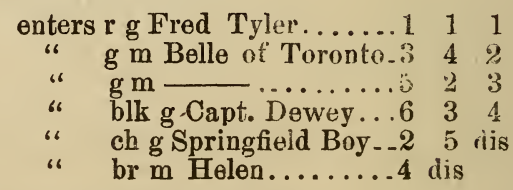

enters $\mathbf{r}$ g Fred Tyler....... $1 \quad 1 \quad 1$

" ch g Springfield Boy_-2 5 iis

" br m Helen........4 dis 
OCT. 19 TH, 1369.

\section{HERDIC PARK, Williamsport.}

OCT. 19.h.-Puise $\$ 1,000$, for horses that have never beaten $3: 00$. Mile heats, best three in five, in harness.

W. H Doble

R. Reamer

H. S. Hitner

E. C. White

J. M. Miller

S. D. Bruce

J. Quinn

R. F. Fawcett

H. Hunt

J. Kreamer

G. N. Belle

F. Perew

J. J. Watson

A. Goldsmith

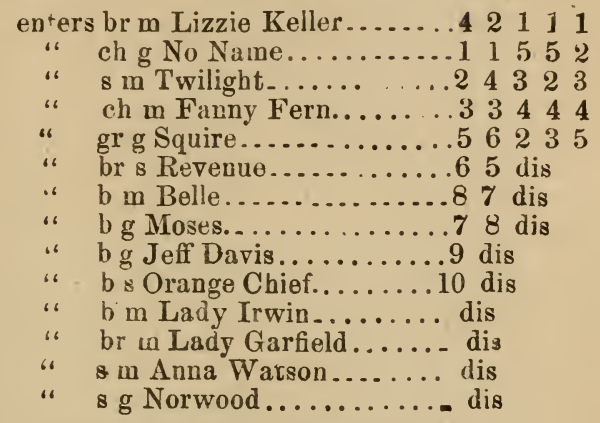

Time-2 40, 2.38 $\frac{1}{2} ; 2.35,2.36 \frac{1}{4}, 2.38$.

OCT. 19тн, 1869.

\section{GERDIC PARK, Williamsport, Penn.}

Oct. 19th.-Purse $\$ 2,000$, for horses that have never beaten 2.33.

Mile heats, best three in five, in harness.
D. Mace
B. Doble
J. Jenniugs
W H. Doble

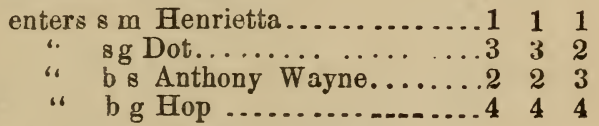
Time-2:35, 2:34衣, 2:35.

OCT. 20TH, 1869.

\section{HERDIC PARK, Williamsport, Penn.}

Oct. 20th.-Purse $\$ 5,(00$, for horses that never have beaten 2.2\%. Mile heats, best three in five, in larness.
B. Mace
D. Mace
J. Turner
J. M. Miller
F. Perew " ch s Byroo..............

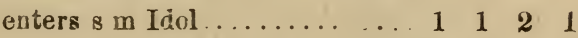

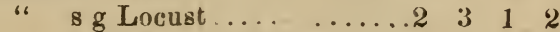

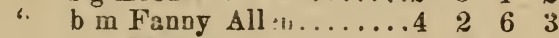
$\begin{array}{llllll}\text { “ b m Mary ............... } 6 \text { 6 } & 6 & 5 & 4\end{array}$
S. R. Clarke " br m Muuntain Maid.....3 44 4 6

Time-2:32⿺, 2:36市, 2:32ł, $2: 35 \frac{1}{2}$. 
OCT. 20TH, 1869.

ST. JAMES COURSE, Smithtown, I. I.

Ocr. 20th.-Purse $\$ 50$. Mile heats, best thres in five, in narness.

L. S. Jones enters b m Lady Jones............. . 111

C. D. Smith " br g Pat Malloy...........2

Time-2:44, 2:46, 2:47.

\section{UNION COURSE, L. I.}

OCT. 20th. -Match $\$ 500$. Mile heats, best three in five, to wagons.

L. S. Sammis enters blk m Eastern Queen....... 1 1 1

H. Howe " b m Lady Wells. ........2 2

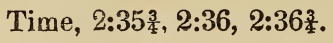

OCT. 20TH, 1869.

HERDIC PARK, Williamsport, Penn.

OCr. 20th.-Purse $\$ 500$, for horses that have never beaten 4.00 . Mile heats, best three in five, in harness.

E. C. White enters ch m Fanny Fern......... 1

H. S. Hitner " $\quad$ s $\mathrm{m}$ Twilight............

J. Kreamer " s m Quaker Girl.......... 3 4 43

W.H. Hudaleston " blk m Rachael..........5 54

H. Hunt “ br g Jeff Devis............ dis

J. C. Delone “ blkg Onward.............. dis

J Quinn " b m Bell................dis

J. $\mathbf{E}$. Hartrauft “ b sa

A Goldsmith " $\mathrm{g} g$ Norwood................. dis

Wyeeff \& Co. " b s Mack ................ dis

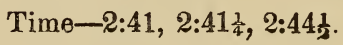

OCT. 21sT, 1869.

HERDIC PARK, Williamsport, Penn.

Ocr. 21 st. - Purse $\$ 1,000$, for horses that have never beaten 2.50 . Mile heats, best three in five, in hrorness.

W H. Doble enters br m Lizzie Keller......... 1 1 1

E. C. White " ch m Fanny Fern...........2 2

F. Perew " b b m Lady Garfield........... 3 ; 3

J. J. Watson "

G. N. Bell " b m Lady Irwin............ 4 4 5

Time, 2:42, 2:44, 2:44. 
OCT. $21 \mathrm{sT}$.

HFRDIC PARK, Williamsport, Penn.

Ост. 21st.-Puree $\$ 1,500$, for all horses that have never beaten 2:42. Mile heats, best three in five, in harness.
J. S. Baker
enters ch g Tom Keller..2 11622311
F. Wagner
R. Reamer
D Mace
W. D. Richards
F. Perew
J. M. Miller
R. F. Fawcett
B. Daniels
S. D. Bruce .

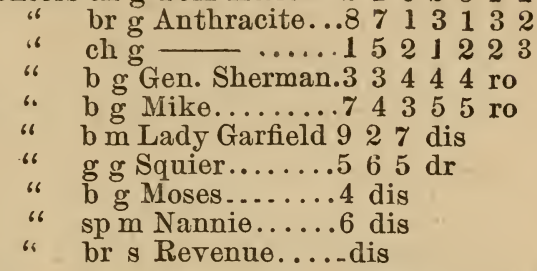

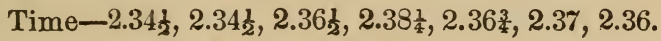

OCT. $21 \mathrm{sT}$.

\section{HERDIC PARK, Williamsport, Penn.}

OCT. 21st. - Purse $\$ 7,000$, for all horses. Mile heats, best three in five, in harness.
B. Doble
enters
b Goldsmith Maid......
B. Daniels

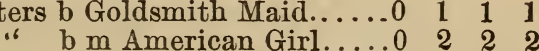

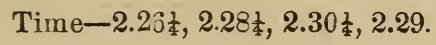

OCT. 22ND.

\section{HERDIC PARK, Williamsport, Penn.}

OCr. 22d.-Purse $\$ 500$, for horses that have never beaten 3:30 Mile heats, best three in five, in harness.

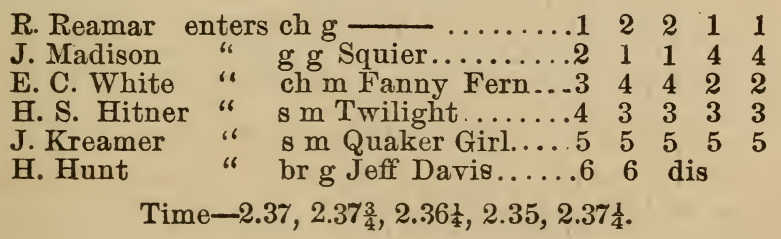

OCT. 22ND.

HERDIC PARK, Williamsport, Penn.

Ост. 22d.-Purse $\$ 2,000$, for horses that have never beaten 2:30. Mile heats, best three in five, in harness. 

E. Scott
B. Doble
D. Mace
enters b $\mathrm{g}$ Lute.

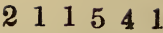
J N. Miller
W. H. Doble
A. Goldsmith
" b g N. B. Palmer.....322 2112
J. Jennings
M. Birdsali
P. Daley
" sm Henrietta.......13342 dis
" b m Mary..........444433 ro
" bgHop............75 $54 \mathrm{dr}$
" b m Lady Whitman..5 766 dr
" b s Anthony Wayne..8 $67 \mathrm{dr}$
" b g Gray John .... -6 dis
" b g Harry D ........9 dis

Time-2.32 $\frac{3}{4}, 232 \frac{3}{4}, 2.31 \frac{1}{2}, 2.31 \frac{3}{4}, 2.33,2.35$.

OCT. $25 \mathrm{TH}$

FASHION COURSE, I. I.

Ocr. 25th.-Match $\$ 1,000$. Mile heats, best three in five, in harness.

B. Doble enters ch $g$ Dot.............

J. Cudney " ch s Hickory Jack......2 1122

Time-2.35, 2.333, 2.33, 2,34.

OCT. 25TH.

FASHION COURSE, $\boldsymbol{L}$. I.

OCT. 25th.-Match $\$ 500$. Mile heats, best three in five, in harness.
J. Murphy
enters ch $\mathrm{m}$ Louise........... 2221
J. Paulding
ch s Burger............. 1 dr

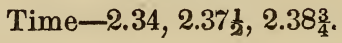

OCT. $25 \mathrm{TH}, 1869$.

\section{PROSPECT PARK COURSE, I. I.}

Ocr. 25th.-Blanket and Hood, for horses owned by members of the Club, that have never beaten 3:00. Mile heats, best tbree in five, to wagons.

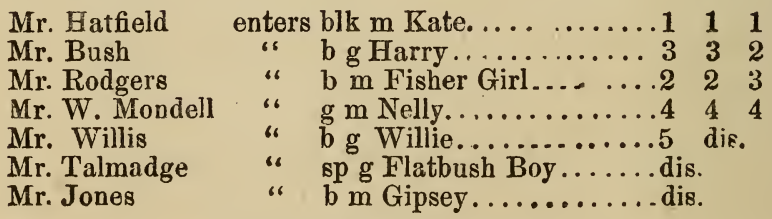

Time-2:44, 2:45, 2:42. 
OCT. $26 \mathrm{TH}, 1869$.

EASTON, Pa.

OCT. 26th.-Purze \$150. Mile heats, best three in five, in harness.

D.6M. Young

J. B. Mettler

S. Hartpence

R. Dempster

J. G. Robbins

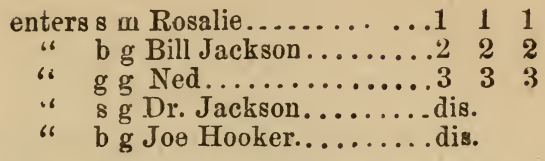

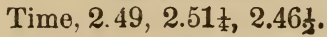

OCT. 26 TH, 1869.

EASTON, Pa.

Oct. 26th.-Purse $\$ 175$, for horses that have never benten 3. 0 . Mile heats, best three in five, in harness.

P. Mauee enters b g Barney Williams ....... 11

J. Turner " s m Twilight............ 32

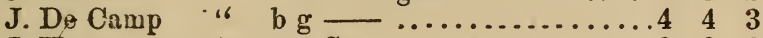

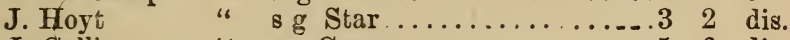

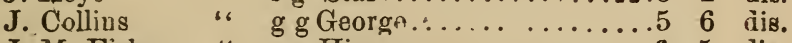

J. M. Fisher " $8 \mathrm{~g}$ Hiram............

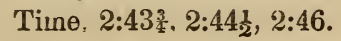

OCT. 26тн, 1869.

EASTON, Pa.

OCT. 26tb.-Purse $\$ 800$, for horses that have never beaten 2:30. Mile heats, best three in five, in harness.

E. R. Smith enters b g Lute ........4444412 1

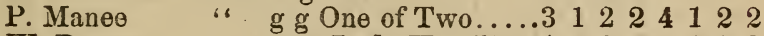

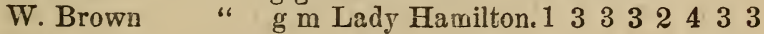

J. Odikirk " blk s Patchen Chief $2 \begin{array}{llllll}2 & 1 & 1 & 3 & 3 & \text { dis. }\end{array}$

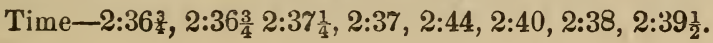

OCT. $2 \%$ TH, 1869.

EASTON, Pa.

Ocт. 27th.-Purse $\$ 200$, for horses that have never beaten 2:50. Mile heats, best three in five, in harness.

A. L. Dostin enters br $g-\ldots \ldots \ldots \ldots 563111$

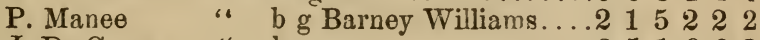

J. De Camp " " b g

B. C. Guerin " s m Rosalie.............. 244555 
J. Hoyt enters s g Star ..........6 3663444

J. M. Fisher " s g Hiram.............444243 ro

J. Turner " s m Twilight..........

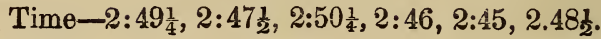

OCT. 28TH, 1869.

EASTON, Pa.

Ocr. 28th. - Purse $\$ 275$, for horses that have never beaten 2:40. Mile heats, best three in five, in harness.

B. C. Guerin enters s m Rosalie.............. 1 1 1

A. L. Dostin "6 b g

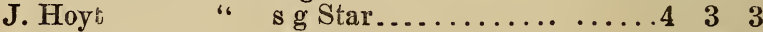

J. De Camp “ s g Sorrell George..........

J. Collins " " s m Lady Whitney.........5 dis

P. Manee “ b g Barney Williams.........ro.

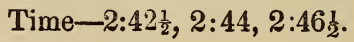

OCT. 28TH, 1869.

EASTON, Pa.

Oct. 28th.-Purse $\$ 1,500$, for all horses. Mile heats, best three in five, in harness.

B. Dobble

J. Turner

S. R. Clarke

$\begin{array}{cccc}\text { enters b g Hotspur............... } & 1 & 1 \\ .6 \quad \text { b m Fanny Allen .......... } & 2 & 2 \\ \text { " br m Mountain Maid...... } & 3 & 3\end{array}$

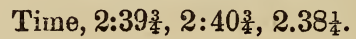

OCT. $28 \mathrm{TH}, 1869$.

FASHION COURSE, I. I.

OCr. 28th.-Match $\$ 1,000$. Mile heats, best three in five, in harness.

J. Lovatt enters ch m Grace B............. 1

A. Patterson . " br m Mystic Maid.............. dis

Time-2:42, 2:36.

ОСТ. 28тн, 1869.

BACIIMORE, Md.

OcT. 28th.-Purse $\$ 300$, for horses that have never beaten 3:00. Mile heats, in harness. 
Mr. Stevens Mr. Sheeler Mr. Murphy Mr. Murphy

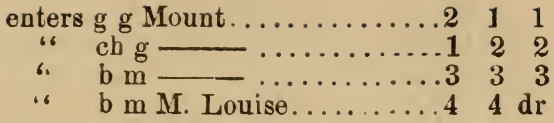

Time-2:43, 2:41 $\frac{1}{2}, 2: 43 \frac{1}{2}$.

OCT. $28 \mathrm{TH}, 1869$

BALTIMORE, Ma.

OCT. 28th.-Purse $\$ 200$, for horses bred in Maryland. Mile heats, in harness.

Mr. Murphy Mr. Johnson

enters br m M. Louise........... 1

. b s N. B. Hayes...........2 2

Time-3:03, 3:02.

OCT. $28 \mathrm{TH}, 1869$.

BALTIMORE, Md.

Ocr. 28th.-Purse $\$ 300$. Mile heats, in harness.

C. F. Fawcett

enters Moses.............2 11

C. A. Murphy

" No Name............ 2 2 2

Time-2:46 $\frac{1}{2}, 2: 42 \frac{1}{2}, 2: 40 \frac{3}{4}$.

OCT. 28TH, 1869.

TROY, N. Y.

OCT. 28th.-Purse $\$ 800$, for horses that bave never beaten 3.0 ). Mile beats, best three in five, in harness:
A. Patterson
enters Soubrette............. 11
T. O. Cunner
J. Dugrey Jr.
E. C. White
D. Jenkins

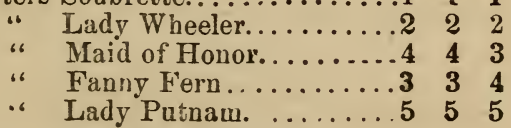

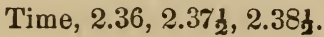

OCT. 28тH, 1869.

TROY, N. Y.

OcT. 28th.-Purse $\$ 1,200$, for horses that have never beaten 2.35. Mile heats, best three in five, in harness. 
M. Roden

D. Mace

E. G. Kaline

J. Cudney enters Prince ..... . . . . $4 \quad 2 \quad 2 \quad 1 \quad 1 \quad 1$

“ $\quad$ Lydia Thompson.... 1112

“ Lolla Montes....... 3 4 $4 \begin{array}{cccc}\mathbf{3} & \mathbf{3} & \mathbf{3}\end{array}$

“ Twilight..........2 $3304 \quad 4 \quad 4$

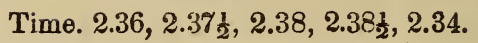

\section{TROY, N. $\mathbf{X}$.}

OCT. 29th.-Purse $\$ 1,000$, for horses that have never beaten 2.40. Mile heats, best three in five, in harness.

E. Hickey

J. Dugrey, Jr.

A. Patterson

S. Reynolủs

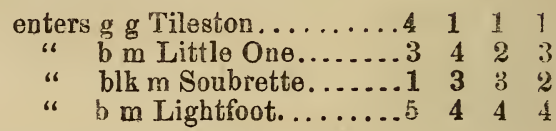

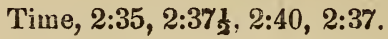

OCT. $29 \mathrm{TH}, 1869$.

TROY, N. $Y$.

Oct. 29th.-Purse $\$ 1,500$, for horses that have never beaten 2.28. Mile heats, best three in five, in harness.

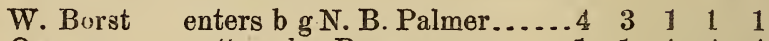

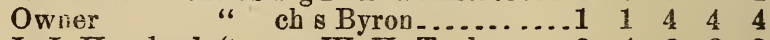

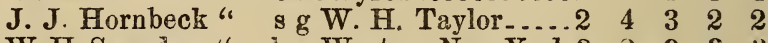

W.H Saunders “ b g Western New York $3 \quad 2 \quad 2 \quad 3 \quad 3$

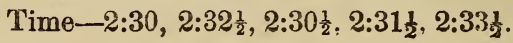

OCT. 29TH, 1869.

FORD'S PARI, Denver, Col.

OCT. 29th.-M Mtch $\$ 500$. Mile heats, best three in five. in harness.

J. Wanless enters b g Prairie Buck .......... 1 1 1

W. R. Furd " b g Shiptimber ............ 2 2 2

Time, $2.41 \frac{3}{4}, 2.41 \frac{1}{2}, 2.43 \frac{3}{4}$.

OCT. 30 TH, 1869.

PEKIN, III.

OCT. 30th-Purse $\$ 100$. Mile heats, best three in five, in har ness. 


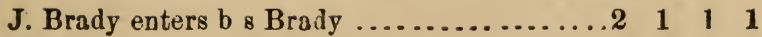

M. Orr “ blkg Gibbo...............

J. Kimball " s g Lumber John............ 3 3 $33 \quad 3$

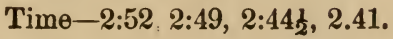

OCT. 30TH, 1869.

TROY, N. Y.

Purse $\$ 2,000$, for all horses. Mile heats, best three in five, in harness.

B. Daniels enters b m American Girl............ 1 1 1

W. Borst " br s Geo. Wilkes................ 2

D. Macs " b m Lucy................. 3 3 3

Time, $232,2.28,2.29 \frac{1}{2}$.

OCT. 30TH, 1869

TROY, N. $\mathbf{Y}$.

EOCT. 30th.-Purse $\$ 1,000$, for horses that have never beated 2.50. Mile heats, best three in five, in harness.

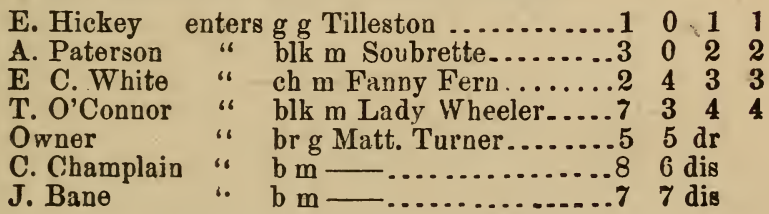

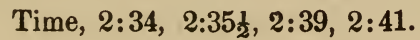

NOV. 1st, 1869.

FASHION COURSE.

Nov. 1st. -Sweepstakes $\$ 600$. Mile heats, in harness.

T. Lynch enters b g Bull of the Woods..........1 1

P. Gavray " b m Jenny Lind...............2 2

M. Kane " g g Gray Eagle................ 3

Time, 3.05, 3.05.

NOV. 2ND, 1869.

DUBOIS TRACK, N. $\mathbf{Y}$.

Nov. 2nd.-Match $\$ 500$. Mile heats, best three in five, under a addle. 


\section{4}

Captain Leary enters blk m Kate McGowan..... 1 1 1 Dr. Ogle

b g Red Rover...........2 22

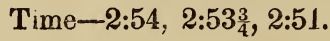

NOV. 2ND, 1869.

RICHMOND Va.

Nov. 2nd.-Purse \$—. Mile heats, in harness.

D. T. Harvey enters blk s Fly Away............

J. E. Paxon " b g Twist...............

Time, 2.47, 2.391 $2.39 \frac{1}{4}$.

NOV. 2ND, 1869.

BAY VIEW PARK, California.

Nov. 2nd.-Match, $\$ 3,000$. Mile heats, best three in five, in harness. Uwners to drive.

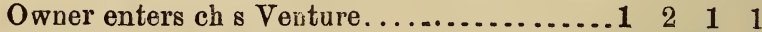

Owner " bs Ajax...................

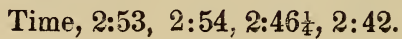

NOV. 2ND, 1869.

MYSTIC PARK, Mass.

Nov. 2nd.-Handicap race. Mile heats, best three in five, in harness.

H Bradley enters b g Capt. Lawrence...3 11 1 1

W H. Woodruff ". b.m Emma..........

$\begin{array}{llllll}\text { J. J. Bowen " blk } m \text { Ida May........ } 4 \text { " } & 3 & 4 & 2\end{array}$

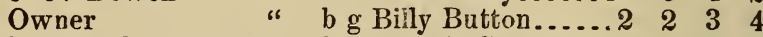

H. Woodruff " b m Jennie Spencer...5 $55 \quad 5 \quad 5$

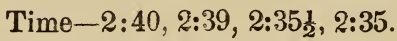

NOV. 3RD, 1869.

MYSTIC PARK, Mass.

Nov. $3 \mathrm{~d}$-Testimonial meeting to professionals. Benefit race. Milea heats, best three in five, in hariess.

M. Carroll

B. S. Wright

A. W: Wooster enters b g N. B. Palmer.....1 2 2 11

" blk m Jessie Wales...2 1130

" ch g McClellan.......3 $3 \quad 2 \quad 2$

Time, 2:34, 2:30, 2:33, 2:33. 
NOV. 3RD, 1869.

WHFEISING, Va.

Nov. 3rd.-Purse $\$ 150$, for horses that have never beaten 2.50. Mile heats, best three in five, 'in harness.

Owner enters Lady Mitchell .... ............... 1

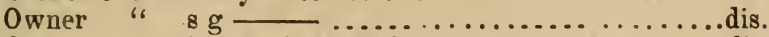

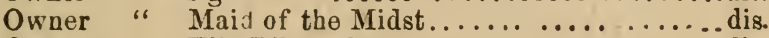

Owner " Ella Ellwood......................dis.

Time, 2:501 .

NOV. 3RD, 1839.

FASHION COURSE, L. I.

Nov. 3rd.-Sweepstakes $\$ 1,200$. Mile heats, best three in five n harness.

J.L. Hornbeck enters s g W. H Taylor......2 $11 \quad 1 \quad 1$

M. Roden

W. Saunders

W. Brown

P.'Manee

‘ b $\mathrm{g}$ Dresden............

" b g Western New York3 $2 \quad 3 \quad 3$

" g m Lady Hamilton...4 $55 \quad 5 \quad 4$

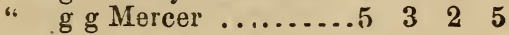

Time, $230 \frac{1}{4}, 2.30,2.33 \frac{1}{4}$, no time.

NOV. 4TH, 1869.

FASHION COURSE, L. I.

Nov. 4th.-Stake $\$ 30$ ), half forfeit. Mile heats, best three in five, in harness.

J. Doty enters b m Topsey................ 1

J. Murphy “ ch m Louise $\ldots \ldots \ldots \ldots \ldots \ldots \ldots 2 \quad 2 \quad 2$

Time, $2.35,2.34$, 2.36 .

NOV. 4 Ta, $1: 69$.

SAY JOSE, Cal.

Nov. 4tb.-Purse \$400. Mile heats, best three in five, in harness.

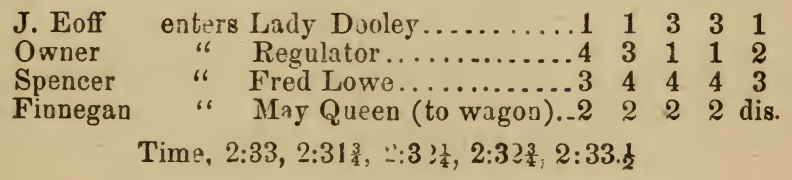


NOV, 4TH.

\section{PROSPECT PARK COURSE, I, I.}

Nov. 4th.-Premium an Afgban valued at $\$ 100$, for horses owned by members of The Prospect Park Association, that have never beaten 3,00. Mile heats, best three in five to wagons.

S. Jackson enters b $g$ Willie........... $4 \begin{array}{llllll} & 2 & 1 & 3 & 1 & 1\end{array}$

T. Jackson " b m Virginia. ......

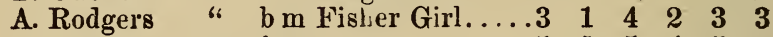

H. Howe "

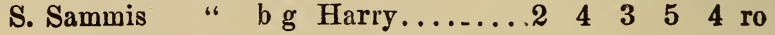

Time 2.41, 2.441, 2.44, 2.48, 2.44, 2.44 .

\section{NOV. 5TH \\ WHEEIING, Va.}

Nov. 5th.-Purse $\$ 200$. Mile heats, best three in five, in harness.

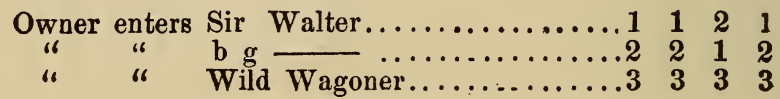

Time-2.43, 2.41, 2.43, 2.41․․․

NOV. 5TH.

MYSTIC PARK, Mass.

Nov. 5th.-Match $\$ 500$. Play or Pay. Mile heats, best three in five in harness.

R. L. Flanders enters $b$ g Dew Drop......... 1 1

H. Bradley " b g Capt. Lawrence.....2 22

Time-2.39, 2 363․ 2.38 .

NOV. 5TH.

MYSTIC PARK, Mass.

Nov. 5th.-Sweepstakes. $\$-$ Mile heats, best three in five.

S. Hays enters br $\mathrm{m}$ Lady Wllkes (in harness). $1 \quad 1 \quad 1$

D. Bigley " b m Kate (to wagon).........2 2

D. Beckler " b in Fanchon (to wagon)....... 33

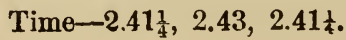


NOV. 5 THI.

\section{FASHION COURSE, L. I.}

Nov. 5th.-Match $\$ 500$. Mile heats, best three in five.

J. Murphy enters b m Fanny Murphy (under sadule)............

W. Lobell enters $\mathrm{z} \mathrm{m}$ Village Girl (to wagou)...2 2

Tiıne-2.37, $243,243$.

NOV. $6 \mathrm{TH}$.

FASEION COURSE, L. I.

Nov. 6th.-Sweepstakes $\$ 300$. Mile heats, best three in five, in harness.

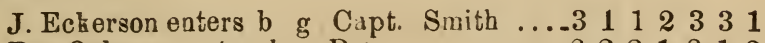

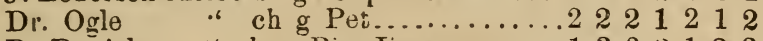

B. Dauiels “ b g Big Jim............

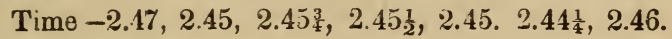

NOV. 6TH.

FASHION COURSE, L. I.

Nov. 6th.-Match $\$ 200$. Mile heats, best three in five, in barness.

J. Doty * b m Topsey..............2 2

Time-2.35, 2.323ㄴ. 2.33! ‥

NOV. 6TH.

EASTON, Pa.

Nov. 6th.-Yatch $\$ 100$. Mile heat3, best three in fire, in harness.

T. Nerwman enters br m Greenwich Maid...2 12211

A. Miller " b in Fanny Fisher.......122122

Time-2.55, 2.50, 252, 2.48, 2.46 $\frac{1}{2}$.

\section{NOV. 9TH. \\ UNION COURSE, L. J.}

Nov. 9th. -Sweepstakes $\$ 100$. Mile heats, best three in five to wagons. 


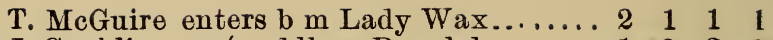

J. Conklin "6 blk m Rosedale........

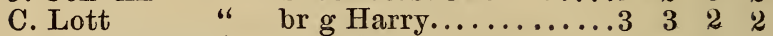

C. Van Winkle" blks Black Crook....... 4 4 44 4

Time $-3.14 \frac{1}{2}, 3.05,3$ 05, 3.03.

NOV. 9TH.

BALTIMORE, Md.

Nov. 9TH.-Purse $\$ 1,000$, for horses that have never beaten 3.00. Mile heats, best three in five, in harness.

J. G. Pfifer enters b g Moses........ 1 \& $3 \begin{array}{lllll}3 & 1 & 2 & 3 & 1\end{array}$

A. J. Hurd " b b Goshen.......2 12222122

W. H. Birch " s g Arion...........

Time-2.36 $\frac{1}{2}, 2.39,2.41,2.42,2.37 \frac{1}{2}, 2.38 \frac{1}{2}, 2.38$.

NOV. 10TH.

BALTIMORE, Md.

Nov. 10th.-Purse $\$ 1,500$. Mile heats, best three in five, in harness.

W. H. Borst enters b g J. J. Bradley $1 \begin{array}{lllllll}2 & 0 & 0 & 2 & 1 & 2 & 1\end{array}$

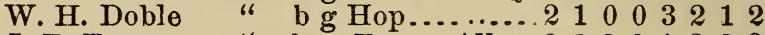

J. E. Turner " b m Fanny Allen.3 $3 \begin{array}{lllllllllll}3 & 3 & 1 & 3 & 3 & 3\end{array}$

W. H. Doble " blk s Star of the

West........................4444

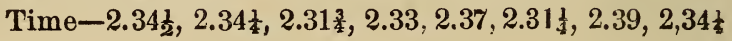

NOV. $10 \mathrm{IH}$.

BALTIMORE, Ma.

Nov. 10th.-Purse $\$ 2,000$. Mile heats, best three in five, in harness.

B. Doble enters b m Goldsunith Maid „...... 1 1 1

W. H. Borst " br s Geo. Wilkes...........2 2

W. H. Doble " b g Hotspur.............. 3 3 3

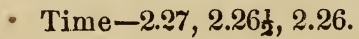

NOV. $11 \mathrm{TH}$.

BALTIMORE, Ma.

Nov. 11tb.-Match $\$ 1,000$. Mile heats, best three in live, in harness. 
$\begin{array}{lllll}\text { B. Doble } & \text { enters s g Dot................ } & 1 & 1 \\ \text { A. Johmson } & \text { br s Patchen, Jr.........2 } & 2 & 2\end{array}$

Time-2.36, 2.34, 2,41.

NOV. 12TH.

HAMPDEN PARK, Mass. harness.

Nov. 12th. -Match $\$ 100$. Mile heats, best three in five, in

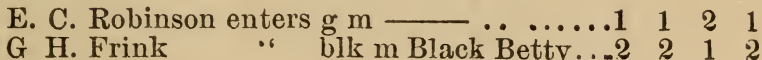

Time-2.53, 2.51, 2.56, 2.52.

NOV. 12th.

\section{MIDDLETOWN, Orange Co., N. Y.}

Nov. 12th.-Purse $\$$ - for county horses. Mile heats, best three in five, in harness.

J. R. Woods enters b m Mills Mare......2 11110021

G. N. Bell " b m Lady Irwin........

Owner " b m Goldsmith Maid. . 33303 ro

W. B. Smith " b m Lady Wheeler....dr

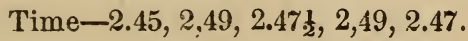

NOV 12 гн, 1869.

\section{FASHION COURSE, I. I.}

No7. 12th. -Sweepstakes $\$ 300$. Mile heats, best three in tre to wagons.

Dr. Ogle

Wm. Lovell

J. Eckerson

enters ch g Pet.............2 111

$\begin{array}{llllll}6 & \text { b g Big Jim............. } & 3 & 3 & \mathbf{2}\end{array}$

" b g Capt. Smith.......3 2203

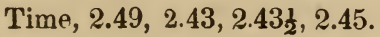

NOV. $12 \mathrm{TH}, 1869$.

FASHION COURSE, I. I.

Nov. 12TH.-Match $\$ 200$. Mile heate, best three in Giro.

J. Murphy enters b m Lady Murphy, (in harness)..1 11

Wm. Lovell " $\quad \mathrm{rm}$ Village Girl (to wagon)....2 2

Time, 2.45, 2.42, 2.53. 
NOV. 13TH, 1869.

\section{UNION COURSE, I. I.}

Nov. 13th.-Match $\$ 200$. Mile heats, best three in five, in harness.

G. Allen enters b g Riley............

S. Sammis " br m Hannah...........

Time, 2.53, 2.52, $2.542 .53,{ }^{\prime} 2.53$.

NOV. 16TH, 1869.

FASHION COURSE, L..I.

Nov. 16th.-Sweepstakes $\$ 200$. Mile heats, best three in five in harness.

Dr. Ogle

J. Martin

H. Casey

Owner

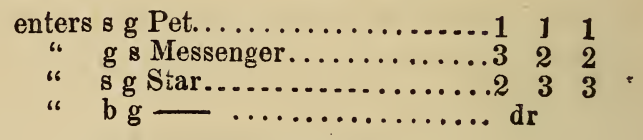

Time, 2.44, 2.48, 2.44 .

NOV. $18 \mathrm{TH}, 1869$.

FASHION COURSE, L. I.

Nov. 18th. -Sweepstakes $\$ 200$. Mile heats, best three in five in harness.

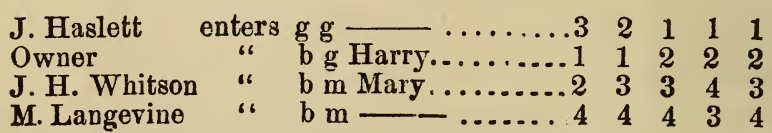

Time, $2.51 \frac{1}{2}, 2.45,2.44 \frac{3}{4}, 2.41_{\frac{1}{2}}, 45$.

NOV. $18 \mathrm{TH}, \mathrm{I} 869$.

\section{UNION COURSE, L. I.}

Nov. 18th.-Match $\$ 500$. Mils heats, best three in five, to wagons.

B. Daniels enters $r$ m Village Girl.......... 1

J. Murphy “ b m Lady Murphy........2 $2 \quad 2$

Time-2.50, 2.50, $2.44 \frac{1}{2}$. 
NOV. 18TH, 1869.

\section{UNION COURSE, L. I.}

Nov. 18th.-Purse \$50. Mile heate, best three in five, in harness.
A. C. Whitson
B. Daniels
L. S. Sammis
J. H. Whitson

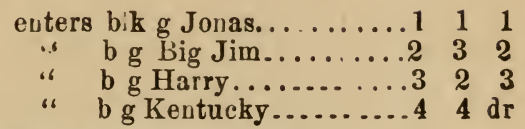

Time, $2: 49 \frac{1}{4}, 2: 49 \frac{1}{2}, 2: 51 \frac{1}{2}$.

NOV. 18TH, 1869.

UNION COURSE, L. I.

Nov. 18th.-Match $\$ 400$. Mile heate, in harness.

G. Allen enters b g Riley............... 1

L. S. Sammis " b m Hannah.............. 2

Time, $3.00,2.56,2.55 \frac{1}{2}$.

NOV. $19 \mathrm{TH}, 1869$.

LAWRENCE, Kansas.

Nov. 19th.-Purse $\$ 100$ for horses that have never beaten 2.45. Mile heats, best three in five, in harness.

E. A. Smith enters b g Billy Weston........... 1

T. H. Clarke " r g Phil .................2 dis

G. P. Wilson " b s Telegraph............... dis

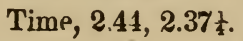

NOV. 22ND, 1869.

FASHION COURSE, I. I.

Nov. 22nd.-Sweepstakes $\$ 150$. Mile heats, best three in fire, in harness.

Dr. Ogle enters b g Red Rover............ 1 1 1

J. Martin “ $g$ \& Messenger................. 3 ( 32

H. Curry “ b m Anna.................

Time, $2.48,2.48,2.48 \frac{1}{2}$. 


\section{2}

NOV. 22ND, 1869.

FASEION COURSE, I. T.

Nov. 22nd.-Purse and stake $\$ 500$. Mile heats, best three in five, to wagons.

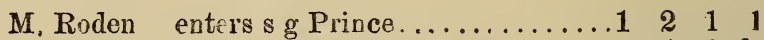

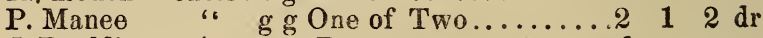

J. Paulding

Time, 2.521 $, 2.51 \frac{3}{4}, 2.43 \frac{1}{2}$.

NOV. $25 \mathrm{TH}, 1869$.

UNION COURSE, L. I.

Nov. 25th. -Sweepstakes $\$ 750$. Mile heats, best three in five, in harness.

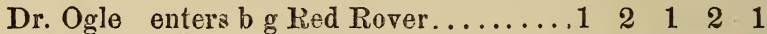

J. Murphy " b b goger.............

M. Roden " b m Kate Mc Gowan..... $3 \begin{array}{lllll}3 & 3 & 3 & 2\end{array}$

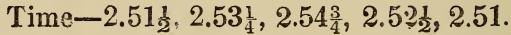

NOV. 30TH, 1869.

UNION COURSE, I. I.

Nov. 30th.-Match $\$ 500$. Mile heats, best three in five, in harness.

H. Howe enters $\mathrm{rm}$ Susey ............... 1

Mr. Jarvis "6 s g Rahway................ 2 2

Time, 2: $18 \frac{1}{2}, 2: 48,2: 49 \frac{1}{4}$.

DEC. $1 \mathrm{sT}, 1869$.

FASHION COURSE, L, I.

Dec. Jst.-Sweepstakes $\$ 300$. Mile heats, best three in five.

J. Murphy enters b m Lady Murphy(in harness).. 1121

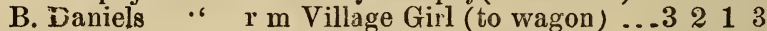

- Dr. Ogle " b g Red Rover (in harness)....2 332

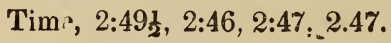




\section{DOUBLG TEAMS.}

JUNE 16TH, 1869.

MYSTIC PARK, MaSs.

June 16 th.-Purse $\$ 2,000$, for double teams. Mile heats, best three in five.

H. A. Hall enters bik $g$ India Rubber Ben

and $b \mathrm{~m}$ Lady Walton..............

W. S. Simmons enters s s Honest Allon and

b g Myron Perry..................

B. S. Wright enters blk s Darkness and

blk m Jessie Wales.............. dis

D. Mace enters b g Twang and $\mathrm{b} g$ Com-

modore Nutt................... dis

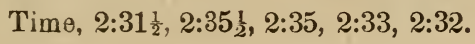

JUNE $25 \mathrm{TH}, 1869$.

NARRAGANSETT PARK, R. I.

June 25th.-Purse $\$ 2,000$, for double teams. Mile heats, best three in five.

D. Maco enters b $g$ Twang and $g \mathrm{~g}$

Mack

B. S. Wright enters b!k m Jessio Wales and blk s Darkness................

F. S. Stevens enters bk s g General

McClellan and b s Garibaldi.........

H. A. Hall enters blk $g$ India Rubber

Ben and $b$ in Rosa ............. 2 dis

W. L. Simmons enters br g Old Put and

ch s Fonest Allen..............dr

Time, $2.33 \frac{1}{2}, 234 \frac{3}{2}, 2.32_{4}^{3}, 2.31 \frac{1}{2}, 2.33,2.33 \frac{1}{2}$.

JULY 29TH, 1869.

RIVERSIDE PARK, Mass.

JuLY 29th.-Purse $\$ 100$, for double teams. Owners to drive. Mile heats, best three in five. 


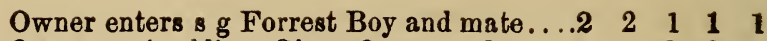
Owner " blk m Gipsy Queen and mate.1 $122 \quad 2 \quad 2$

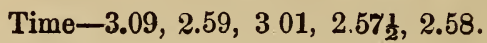

SEPT. 15TH, 1869.

BINGHAIIPTON, N. $\mathbf{Y}$.

SEPT. 15th.-Purse $\$ 500$, for double teams, owned in Binghampton. Mile heats, best three in five.

J. Bigsby

J. O'Hara

W. H. Barber

H. L. Robinson enters George and Bigsby Colt.1 2211

" Ned and Deacon........ 31122

‘. Billy and Barber Mare_2 dis.

“ Gray Mares..........4 dis.

Time, $3.08 \frac{1}{2}, 2.59 \frac{3}{4}, 2.57 \frac{1}{4}, 3.01 \frac{1}{2}$, 一.

SEPT. 16TH, 1869.

PROSPECT PARK COURSE, I. I.

SEPT. 16th.-Purse $\$ 1,500$, for double teams. Mile heats, best three in five.

W. H. Borst enters blk m Jessie Wales and b s

Honest Allen........................

H. A. Hall enters br g India Rubber Ben and

b m Lady Walton .................... $3 \begin{array}{llll} & 2 & 3 & 3\end{array}$

M. Roden enters b g Commodore Nutt and b g

David Bonner................... ro.

Timo-2.313, $2.31 \frac{3}{4}, 2.35 \frac{1}{4}, 2.35$.

SEPT. 30TH, 1869.

COLD SPRING COURSE, Milwaukee, Wis.

SEPT. 30th.-Purse $\$ 250$, for double teams. Mile heats.

L. C. Chase enters India Rubbber and mate..2 11

O. A. Hickock " Blonde and Edna......... 32

J. Demas “ Vulcan and Lottie Clark..1 dis.

Time-2:36, 2:31ћ, 2:35ł.

SEPT. 30TH, 1869.

MYSTIC PARK, Mass.

SEPT. 30th.-Purse $\$ 1,000$, for double teams. Mile heats, bent three in five. 
W. H. Borst enters \& s Honest Allen and blk m Jessie Wales.......................... 11

J. J. Bowen enters blk $g$ India Rubber Ben and b m Lady Walton........................ 322

H. Bradley enters blk $g$ Black Harry and $\mathrm{s} m$

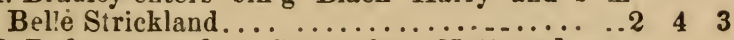

M. Roden enters b g Commodore Nutt and s $g$ Sorrell John.......................4 34

Time-2.31, 2.32, 2..9․․․

OCT. 5TH, 1869.

NARRAGANSETT PARK, R. I.

OcT. 5th.-Purse $\$ 2,000$, for double teams. Mile heats, bes three in five.

H. Bradley enters blk g Black Harry and s m

Belle Strickland... .................2 11 1 1

W. H. Borst enters s s Honest Allen and blk $m$

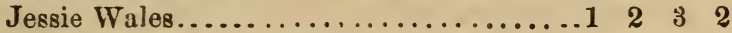

D. Mace enters b m Lydia Thumpson and s $\mathrm{m}$

Idle.......................... $34{ }^{4} \quad 2 \quad 3$

J. J. Bowen enters blk g India Rubber Ben and

b m Lady Walton ................... $4 \begin{array}{llll}3 & 4 & 4\end{array}$

W. S. Briggs enters b s Garibaldi and blk s

Darkness. .................... dis

M. Roden enters b g Commodors Nutt and

s g Sorrell John................... dis

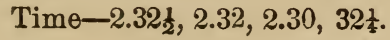

OCT. 7TH, 1869.

DOYLESTOWN, Pa.

Ocr. 7th.-Purse $\$ 30$, for Double Teams. Mile heats.

J. Peters enters Gray Mare and Black Stallion.1 1

C. S Alkinson 'Jack West and Mate........3 2

L. B. Lazalere " Buckeye Maid and Fairy.....2 3

Time-3.10, 2.53년

OCT. $15 \mathrm{TH}, 1869$.

DEXTER PARK, Chicago.

OcT. 15th. - Purse $\$ 300$, for Double Teams. Mile heats, best three in five. 


\section{6}

O. A. Hickock enters Blonde and Mate......2 23131

J. Rockey

J. Demas

Time, 2:37, 2:38 $\frac{1}{2}, 2: 36 \frac{1}{2}, 2: 37 \frac{1}{2}, 2: 42,2.34$.

OCT. $16 \mathrm{TH}, 1869$.

BUCKEY COURSE, Cincinnati, O.

OCT. 16th.-Purse $\$ 500$, for Double Teams. Mile heats, best three in five.

M. Smith enters $s \mathrm{~g}$ Frank and br $g \mathrm{~g}$

Grapevine.......................

R. D. Harshman enters g m Dayton Belle and

ch g Glencos........................ 121212

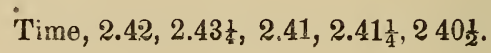





\section{TIFTANY \& C0.,}
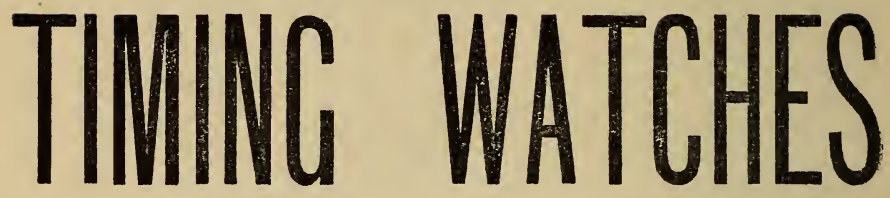

AND

\section{CHRONOGRAPHS.}

The TIFFANY \& CO., TIMER combines all the qualities of a perfect time keeper with the most simple and perfect construction for timing purposes.

The second hand always starts from the figure 12 , thus showing the exact time at sight, avoiding all calculation and possibility of dispute.

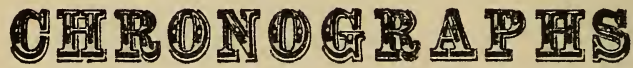

for the stand, both plain and with ink reservoirs; the latter by touching a spring, without stopping, marks the time on the face of the dial, of quarter, half-miles, etc.

Also, a large assortment of ONE-QUARTER, ONE-FIFTH, and INDEPENDENT-SECOND Watçhes, by Charles Frodsham (for whom we are sole agents in New York), Jules Jurgensen, and other makers.

\section{0 \& 522 BROADWAY.}




\section{J N D E X .

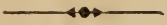

Aron, N. Y...................... $82,8 \gamma$

Abbey Park, St. Louis, Mo.................... 98 Association Park. Detroit, ................... 14, 15, 17 Buckeye Course, Cincinnati, O. 14, 16, 17. 18, 19,20, 29, 107, 108, $109,111,113,136$

Burlington, Vt...................... 31, 85, 86 Buffalo Driving Park, N. Y....... 34, 38, 39, 45, 48, 49, 50, 51 Bingbamton, $\mathbf{N} . \mathbf{Y} \ldots \ldots \ldots \ldots \ldots \ldots \ldots \ldots, 42,79,83,84,134$

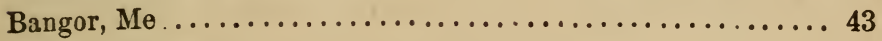

Brattleboro, Vt.......................... 56

Bay View, Cal. ........................ 80, 124

Belvidere, N. J........................... 111

Baltimore, Md. ................... 120, 121, 128

Butterfield Course, Utica ..................... 38

Clinton, Iowa. .................... 25, 61, 62

Cincinnatti, Ohio........................ 47

Copake, N. Y. . . . . . . . . . . . . . . . . . . 62

Cambridge, $\mathrm{N} . \mathrm{Y} \ldots \ldots \ldots \ldots \ldots \ldots \ldots \ldots \ldots \ldots \ldots \ldots \ldots . \ldots \ldots$

Cannastota, N.Y.........................64, 67

Cold Spring Park, Milwaukee.........48, 95, 96, 98, 100, 134

Cocheco Driving Park, Dover, N. H. .......... 54, 56, 58

Citiz «n's Park, Woonsocket, R. I . . . . . . . . . . . . 89, 90

Dexter Pảrk, Chicago, Ill. 7, $] 9,37,38,45,88,91,108,109,113,135$

Doty's. " Poughkrepsie................25, 28, 29

Dabbury, Conn...................... 78

Detroit, Mich............................27

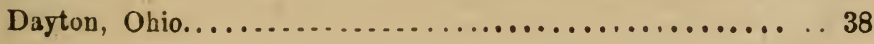


Dubuque, Iowa.................. 45, 108, 110, 112

Dutchess Co.,N. Y.................... 80, 83, 85

Dedham, Mass............................ 91

Doylestown, Pa..................... 103, 105, 135

Duboi's Track, N. Y., City...................... 123

Ellicottsville, Pa.......................... 30, 32

Easton, $\mathrm{Pa} \ldots \ldots \ldots \ldots \ldots \ldots \ldots$. . 36, 48, 75, 86, 119, 120, 127

Erie, Pa............................. 53, 94

Fashion Course, L. I. .. 20, 33, 34, 36, 38, 40, 46, 52, 89, 100, 118 $120,123,125,127,129,130,131,132$

Ford's Park, Denver, Col............. 37, 40, 106, 107, 122

Frai klin Co., Ohiø........................ 70

Forrest City Park, Portland, Me............... 72

Golden Gate Park, San Francisco, Cal........... 21, 39, 52

Grand Rapids. Mich.................... 22, 23, 25

Goshen, N. Y.......................... 30

Great Falls, N. H..................... 35, $\mathbf{3 6}$

Glenbrook, Cal......................... 55

Glenwrod, Iowa........................ 113

Hamilton, C. W.......................... 23

Hamtramck Course, Detroit, Mich........... 77, 80, 84, 88

Hartford, Conn...................... 33, 92, 114

Highland Park Course, Newburgh, ... .............. 9

Hawkeye Course, Clinton, Lowa.$\ldots \ldots \ldots \ldots \ldots \ldots \ldots 23$

Hurstsville, N. Y.......................... 65

Hampton Park, Springfield, Mass..... 58, 59, 60, 61, 62, 92, 129

Hampshire Yark, Amherst, Mass................... 96, 97

Herdic Park, Williamsport, Pa............. 115, 116, 117

Indianapolis, Ind.................. 17, 20, 27

Jamestown, N. Y..................... 59, 110

Knox, Park, Me........................... 31

Leavenworth, Kansas.................... 15, 34

Lexington, Ky. ......................... 108 
Lawrence, Kansas ...................... 44, 131

Lancaster, $\mathrm{Pa} . \ldots \ldots \ldots \ldots \ldots \ldots \ldots \ldots \ldots \ldots \ldots$

Lowell, Mass ........................... 91

Mystic Park. Medford, Mass. 10, 11, 12, 21, 24, 25, 31, 32, 39, 43, $44,57,64,75,93,94,96,99,113,114,124,126,133,134$

Magnolia Course, New Orleans, La................. 18

Manchester, N. H.............. 29, 49, 50, 51, 74, 95

Middletown, N. Y.... $\quad \ldots \ldots \ldots \ldots \ldots \ldots .59,60,61,129$

Mt. Vernon, Ohio.......................... 64

Mendota, Ill.......................... 74

Mrricksville, Mass......................... 93

Middlesex, “ $\quad \ldots \ldots \ldots \ldots \ldots \ldots \ldots \ldots \ldots \ldots \ldots, 107$

Marietta, Ohio............................ . 111

Narragansett Fark, Cranston, R. I. 13, 16, 18, 19, 20, 68, 101, 102, $103,105,106,133,135$

Nashville, Tenn............................ 32,33

National Course, Washington, D. C:............... 75

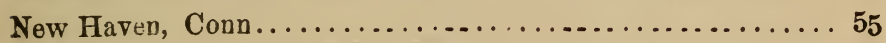

Newburgb, N. Y....................... 65,6667

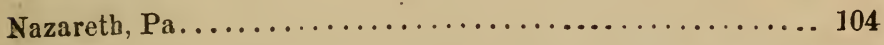

Northampton. is ass......................... 104. 105

Omaha Park, Omaha, Neb................ 26, 41, 45

Olentangy Park, Columbus, O............. 53, 54, 56, 86

Oil City, Pa........................... 52, 53

Prospect Pa1k, L. I..... 34, 62, 64, 75, 76, 82, 87, 118, 126, 134

Portsmiuth, N. H......................... 47

Point Breeze Park, Phila., Pa.......... 63, 65, 68, 69, 73, 81

Paterson, N. J........................ 78

Pittsfield, Ill............................. 112

Pekin, Ill............................... 122

Rensselaer Park, Troy, N. Y .............21, 22, 24, 34

Riverside Park, Brighton, Mass. 8, 9, 42, 46, 47, 54, 63, 90, $92,93$. 
Rittersville. $\mathbf{P a}$

PAGR

Rock Island, IIl

24

Reading, Pa...

70,71

Riverhead. I. I. . $76,79,82,95,96,93,99,100$

Riding Park Course, Manchester, N. H 104

Richmond Va". 46

Springfield, Mass 124

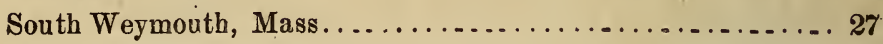

Syracuse, N. Y..................... 28, 43, 67, 70

Suffolk Park, Phila., Pa $\ldots \ldots \ldots \ldots \ldots \ldots$ 39, 43, 48, 57, 63

Saratoga, $N$. Y........................ 47, 58

St. Catharines, C. W.................... 57

St. Lawrence Co., N. Y............... 73, 74, 78, 81

Salem, N. J............................ 97

San Jose, Cal ........................ 103, 125

Smithtown, L. I. .......................... 116

Troy, N. Y....................... 12I, 122, 123

Union Course, L. I... 4, 5, 6, 7, 33, 34, 37, 41, 43, 78, 79, 116, 127, 130, 131, 132

Unionville, Orange Co, N. Y $\ldots \ldots \ldots \ldots \ldots \ldots \ldots \ldots \ldots 25$

Utica, N. Y........................ 27, 57

Union Park, Sacramento, Cal.............. 68, 69, 73

Urbana, Ill........................ 41, 42, 44

Vallejo, Cal............................... 12

Vernon, Orange Co., N. Y...................... 110

Watertown, N. Y........ 12, 13, 15, 16, 18, 52, 102, 103, 106

Worcester, Mass......................... 28, 37

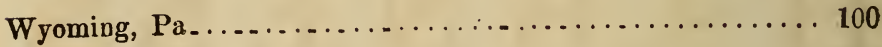

Wavesley, N. J............................ 110

Wheeling Va......................... 125, 126

Ypsilanti, Mich........................... 24 


\section{DUBOIS' WAG0N.}

\section{PETER DUBOIS' SON,}

Carriage Manufacturer FIRST CLASS WORK ONLY.

\section{TRLOK SULKEESS \& SKELETON WAGONS}

MADE A SDECIALTY,

AND ALWAYS ON HAND AT ANY MOMENT.

No, 202 GREENE STREET, INEW YORIK CITY.

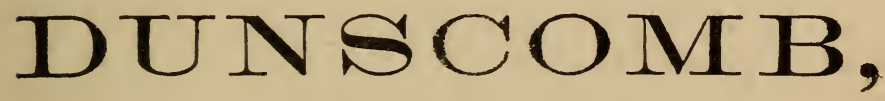

MANUFACTURER OF

Pleasure \& Trotting

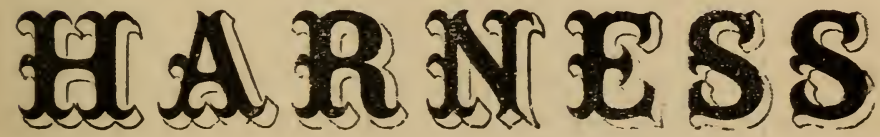

No. 7 Great Jonès Street,

NEW YORK. 




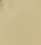



8 



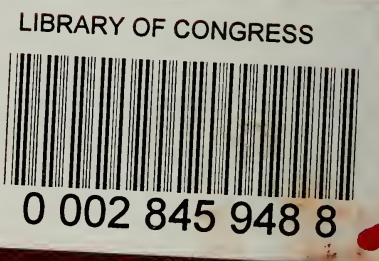

